NBER WORKING PAPER SERIES

\title{
IDENTIFYING AMBIGUITY SHOCKS IN BUSINESS CYCLE MODELS USING SURVEY DATA
}

\author{
Anmol Bhandari \\ Jaroslav Borovička \\ Paul Ho \\ Working Paper 22225 \\ http://www.nber.org/papers/w22225 \\ NATIONAL BUREAU OF ECONOMIC RESEARCH \\ 1050 Massachusetts Avenue \\ Cambridge, MA 02138 \\ May 2016
}

We thank Bryan Kelly, Monika Piazzesi, Tom Sargent, Martin Schneider and Balint Szoke for helpful comments, and Mathias Trabandt for sharing codes with us. The views expressed herein are those of the authors and do not necessarily reflect the views of the National Bureau of Economic Research.

NBER working papers are circulated for discussion and comment purposes. They have not been peer-reviewed or been subject to the review by the NBER Board of Directors that accompanies official NBER publications.

(C) 2016 by Anmol Bhandari, Jaroslav Borovička, and Paul Ho. All rights reserved. Short sections of text, not to exceed two paragraphs, may be quoted without explicit permission provided that full credit, including $\odot$ notice, is given to the source. 
Identifying Ambiguity Shocks in Business Cycle Models Using Survey Data

Anmol Bhandari, Jaroslav Borovička, and Paul Ho

NBER Working Paper No. 22225

May 2016

JEL No. C11,C63,D81,D84,E24,E32

\begin{abstract}
$\underline{\text { ABSTRACT }}$
We develop a framework to analyze economies with agents facing time-varying concerns for model misspecification. These concerns lead agents to interpret economic outcomes and make decisions through the lens of a pessimistically biased 'worst-case' model. We combine survey data and implied theoretical restrictions on the relative magnitudes and comovement of forecast biases across macroeconomic variables to identify ambiguity shocks as exogenous fluctuations in the worst-case model. Our solution method delivers tractable linear approximations that preserve the effects of time-varying ambiguity concerns and permit estimation using standard Bayesian techniques. Applying our framework to an estimated New-Keynesian business cycle model with frictional labor markets, we find that ambiguity shocks explain a substantial portion of the variation in labor market quantities.
\end{abstract}

Anmol Bhandari

Department of Economics

University of Minnesota

bhandari@umn.edu

Jaroslav Borovička

Department of Economics

New York University

19 W. 4th Street, 6th Floor

New York, NY 10012

and NBER

jaroslav.borovicka@nyu.edu
Paul Ho

Princeton University

pho@princeton.edu 


\section{Introduction}

Survey data on households' expectations about future macroeconomic outcomes reveal significant pessimistic average biases and comovement of these biases at business cycle frequencies. In this paper, we present a theory where such pessimism stems from households' concerns that the underlying model they use for decision-making is potentially misspecified. In doing so, we depart from the rational expectations assumption and replace it with a tightly specified framework that links households' decisions and expectations adjusted for plausible misspecification fears. This departure is disciplined using data on macroeconomic variables along with survey data on households' expectations. We use this framework to quantify the magnitude and economic channels through which misspecification concerns affect aggregate outcomes.

Our theoretical foundation is an extension of the robust preference model of Hansen and Sargent (2001a,b). Agents endowed with robust preferences are concerned that the particular model they view as their 'benchmark' model of the economy may be misspecified. Instead of only using the benchmark model, they consider a set of models that are statistically hard to distinguish from the benchmark model. The concerns for model misspecification lead them to choose the model from this set that delivers the lowest utility. This 'worst-case' model is then the basis for their decisions, as in the utility-minimizing prior in the multiple prior framework of Gilboa and Schmeidler (1989) and Epstein and Schneider (2003). The robust preference framework thus represents a particular form of ambiguity aversion.

We extend this robust preference framework to allow the agents to be exposed to shocks to their ambiguity concerns. The time-variation in ambiguity concerns induces fluctuations in agents' worst-case beliefs and endogenously affects equilibrium dynamics. While our extension delivers a more flexible specification of the time-variation in the worst-case model, it still tightly restricts the beliefs across alternative states in a given period. Agents fear outcomes with adverse utility consequences and overweight their probabilities in a specific way.

In order to identify the variation in the worst-case model empirically, we assume that agents' forecasts in the survey data are based on their worst-case model. Our theoretical framework yields directly testable predictions about the mean distortions and comovement of these forecasts under the worst-case model. Specifically, the model predicts a one-factor structure for the survey forecasts of macroeconomic variables, with loadings determined by the covariance of these variables with shocks that have adverse utility consequences. These cross-equation restrictions also allow us to distinguish fluctuations in ambiguity concerns from alternative specifications of subjective beliefs.

We show that household forecasts for key macroeconomic variables in the University of Michigan Surveys of Consumers are indeed significantly pessimistically biased, with a discernible business cycle component. We start by estimating a vector-autoregression (VAR) that embeds household survey data, explicitly restricting the belief distortion (or wedge) between the worst-case model and the data-generating probability measure. A common component of these belief distortions in alternative survey answers identifies a latent factor that captures the time-variation in the worstcase model, and its impact on observable macroeconomic quantities. 
We then combine the robust preference framework and the survey data in a dynamic stochastic general equilibrium model with frictional labor markets, sticky prices and a monetary authority that follows an interest rate rule. We estimate this model using Bayesian methods and study the quantitative role of the ambiguity shocks in the dynamics of the labor market and the comovement of macroeconomic variables.

The results from the VAR and structural models show a common pattern. The worst-case belief is identified from the common variation of the biases in survey answers, and it explains a significant amount of variation in these biases. Ambiguity-averse households interpret high unemployment, low GDP growth and high inflation states as particularly adverse to their utility, and overweight worst-case probabilities of those states substantially.

An adverse ambiguity shock also has significant contractionary effects, propagated particularly strongly through the labor market. In the labor market with search and matching frictions, creation of new matches and hiring depend on the assessment of the future surpluses generated in a new match. An increase in ambiguity concerns leads to a more pessimistic evaluation of future surpluses and therefore to lower match creation, which increases unemployment and decreases output. Fluctuations in ambiguity concerns effectively act as variation in the risky component of the stochastic discount factor, providing a testable structural explanation of the discount rate shock in Hall (2015) linked to observable survey data.

On the technical side, we develop a series expansion technique that incorporates the impact of time-varying ambiguity concerns in the first-order approximation of the model. The main challenge is that the worst-case model distortion arises endogenously and needs to be computed jointly with the equilibrium dynamics, as agents overweight states with low utility realizations. The approximation method leads to a tractable linear solution for the equilibrium dynamics with a role for ambiguity shocks that can be estimated using standard Bayesian techniques.

The paper contributes to the growing literature that quantitatively assesses the role of ambiguity aversion in the macroeconomy, building on alternative decision-theoretical foundations by Gilboa and Schmeidler (1989), Epstein and Schneider (2003), Klibanoff et al. (2005, 2009), Ju and Miao (2012), Hansen and Sargent (2001a,b), Strzalecki (2011) and others. Applications to macroeconomic models include Cagetti et al. (2002) and Bidder and Smith (2012). For a survey of applications in finance, see Epstein and Schneider (2010).

Perhaps the closest to our paper is the work by Ilut and Schneider (2014) and Bianchi et al. (2014), who utilize the recursive multiple-prior preferences of Epstein and Schneider (2003). The main difference between their approach and ours to model ambiguity aversion is how the set of potential misspecifications is parameterized in each case. The multiple-prior framework does not restrict the relative magnitudes of individual shock distortions under the worst-case model, and thus introduces a heavier burden on identification through observable data. In our setting, exposures of household continuation values to the underlying shocks endogenously determine the distortions. These exposures are pinned down by cross-equation restrictions that arise from optimizing behavior of forward-looking agents and impose consistency between the worst-case model and implied 
continuation values. We explicitly characterize these restrictions and utilize them in our estimation and identification of ambiguity shocks. Another difference is that we use data on cross-sectional average distortions measured in household survey answers, for which our theory has direct quantitative predictions, as a source of identification of the ambiguity shocks. Ilut and Schneider (2014) instead use the forecast dispersion as a proxy for confidence and show an empirically plausible relation of this measure to the notion of ambiguity aversion. Despite these differences, we view both approaches as complementary.

The paper is organized as follows. Section 2 describes key empirical findings from the survey data and discusses empirically testable predictions that distinguish ambiguity shocks from other potential explanations of the belief biases. In Section 3, we estimate a latent factor model that captures the time-variation in the common component of the belief biases. Motivated by these findings, we introduce our extension of the robust preference framework in Section 4, link the implications of the theory to the belief biases in survey data, and develop a tractable solution technique for approximating the equilibrium dynamics. Section 5 is devoted to the construction and estimation of the structural business cycle model that embeds robust preferences, and in Section 6 , we discuss implications of the findings and the role of ambiguity shocks in business cycle dynamics. Section 7 concludes. The appendix contains detailed derivations of the developed approximation method, description of the data, estimation details, and further results and robustness checks.

\section{Survey expectations}

We start by analyzing data on households' expectations from the University of Michigan Surveys of Consumers (Michigan Survey). These surveys collect answers to questions about the households' own economic situation as well as their forecasts about the future state of the economy. Specifically, we focus on the forecasts of future inflation, unemployment rate and the Index of Consumer Expectations, which we use as a proxy for GDP growth forecast. A detailed description of the construction of the data and additional statistics are provided in Appendix C.

We are interested in deviations in these survey answers from rational expectations forecasts. The construction of these belief wedges necessarily requires taking a stand on how to determine the probability measure that generates the data. We assume that the Survey of Professional Forecasters (SPF) provides unbiased estimates for the variables we study. Ang et al. (2007), Croushore (2010), Faust and Wright (2013) and others document that professional survey forecasts systematically outperform other forecasting methods. ${ }^{1}$ We also prefer the SPF forecasts to rational forecasts generated in our model because potential misspecification in the constructed model would systematically bias the measurement of the belief wedges, a critical input to our analysis. Nevertheless, as a robustness check, we construct these wedges in Appendix G using several alternatives that also

\footnotetext{
${ }^{1}$ While some studies report modest biases in SPF forecasts, these biases are an order of magnitude smaller than those we find in household surveys, and not robust to the chosen time period. See, e.g., Elliott et al. (2008) and Capistrán and Timmermann (2009), who rationalize these biases by assuming forecasters with asymmetric loss functions.
} 

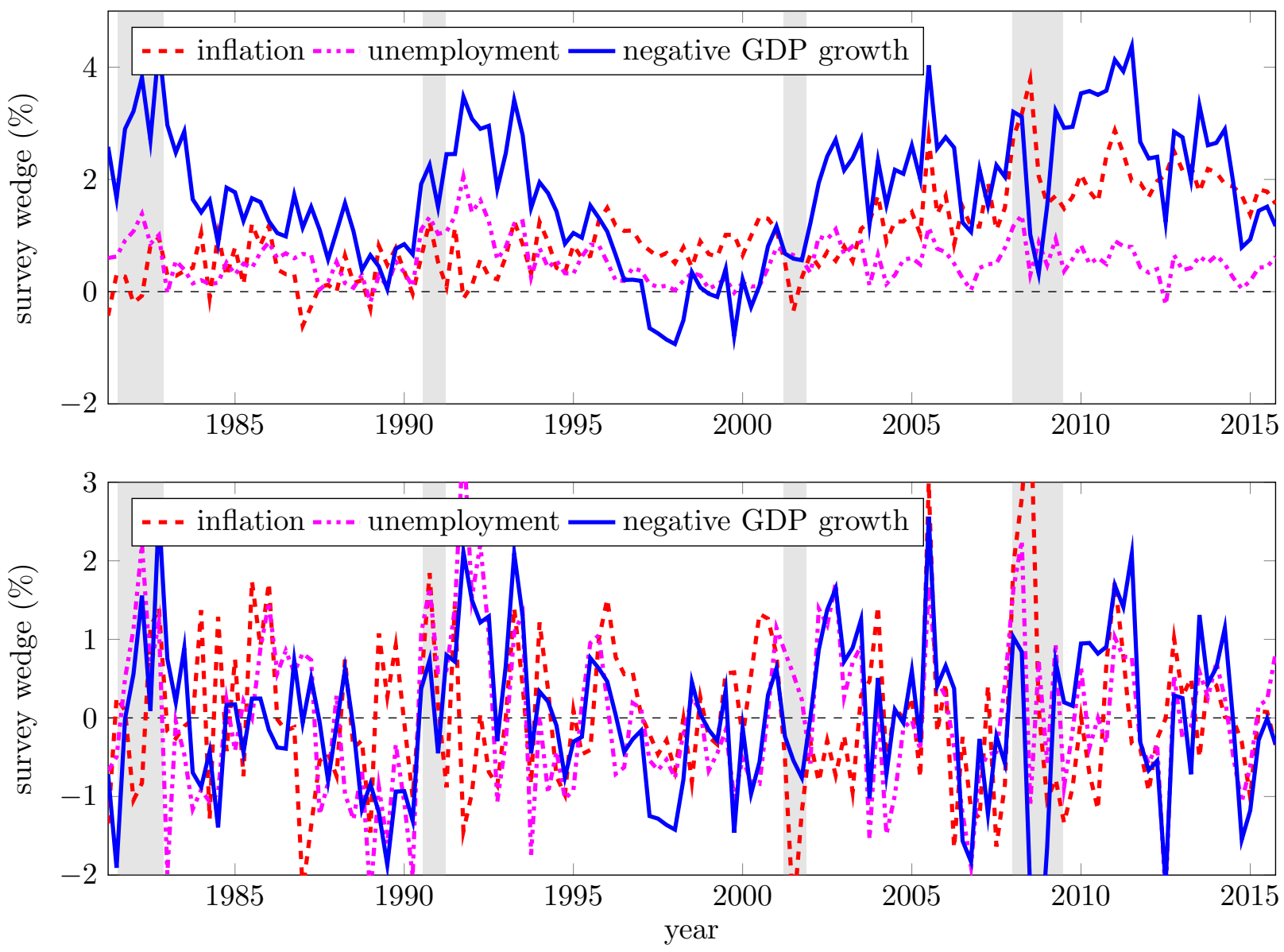

Figure 1: Difference in survey expectations between the Michigan Survey and Survey of Professional Forecasters. Top panel original data, bottom panel HP-filtered and standardized. GDP growth forecast for the Michigan Survey is constructed using a projection on the Index of Consumer Expectations, and the GDP growth wedge is plotted with a negative sign. Details on the construction of the data series are in Appendix C. NBER recessions shaded.

use model-implied forecasts. We show that for each variable these wedges are highly correlated across alternative measurements and yield similar results.

Figure 1 shows the differences in survey expectations between the Michigan Survey and the Survey of Professional Forecasters for inflation, unemployment and GDP growth. The survey expectations are mean one-year ahead expectations in the survey samples. The Michigan Survey does not contain a question about GDP growth, and we therefore proxy it by projecting GDP growth on the Index of Consumer Expectations (ICE) constructed from survey answers. We detail the construction of the time series in Appendix C. Table 5 in Appendix $\mathrm{C}$ summarizes the moments and correlations for the belief wedges.

The top panel of Figure 1 reveals that households' expectations are systematically pessimistically biased - relative to professional forecasters, households overpredict future unemployment 
and inflation, and underpredict GDP growth (with the exception of the boom period during the late 1990s). The average belief wedge over the sample period for the inflation, unemployment and GDP growth wedge is $1.01 \%, 0.55 \%$ and $-1.73 \%$, respectively. ${ }^{2}$

Moreover, despite a substantial amount of noise, the three time series for the belief wedges have a common business cycle component and are statistically significantly correlated. The correlation coefficient for the unemployment and GDP growth wedges is -0.63 , while the correlation between the inflation and GDP growth wedges is -0.32 , with a standard error of 0.07 and 0.08 , respectively. The comovement over the business cycle can be visually confirmed in the bottom panel of Figure 1 that plots HP-filtered and standardized data. All three variables are significantly correlated with the business cycle, measured both using realized GDP growth and the output gap (see Table 5 in Appendix C).

While the unemployment rate wedge appears small relative to the other wedges, this is consistent with a low volatility of the innovations to the realized unemployment rate. The large magnitude of the inflation wedge, in particular in the post-2006 period, is consistent with the findings of Coibion and Gorodnichenko (2015) and others. The fluctuations in the GDP growth wedge may appear large, peaking at $-4.37 \%$ in 2011Q3, in the quarter of the U.S. debt ceiling crisis. ${ }^{3}$ These large fluctuations ask for additional scrutiny, especially given that the Michigan forecast is constructed using the projection on the ICE index. First, given that GDP growth is volatile, it is not surprising and in fact consistent with our theory that households report larger pessimistic biases. Second, Figure 9 in Appendix $\mathrm{C}$ shows that while the constructed household forecast is systematically pessimistically biased, it exhibits very similar business cycle patterns as the actual GDP growth and the SPF forecasts. Finally, our theory predicts that the ratio of the mean of the belief wedge relative to its time-series volatility should be constant across responses for different variables. Table 5 in Appendix $\mathrm{C}$ shows that these ratios for the empirically measured belief wedges are in fact in the very tight range of 1.28-1.47, despite the fact that they are all constructed from independent data. This evidence is reassuring regarding the plausibility of the constructed wedges.

\section{$2.1 \quad$ Forecast dispersion}

Our theoretical framework formalizes the notion of pessimistic belief distortions through the structure of the robust preference model. The common component of the three belief wedges from Figure 1 identifies the fluctuations in the worst-case model of economic agents. We embed the belief distortions in a representative agent framework, which provides a justification for using average forecasts as a measure of subjective expectations in the model.

Mankiw et al. (2003), Bachmann et al. (2012) and others use measures of cross-sectional forecast dispersion as a proxy for economic uncertainty. This proxy is typically based on the presumption that a higher dispersion is indicative of more difficulty in estimating the forecast distribution,

\footnotetext{
${ }^{2}$ The average bias in the SPF data for the three variables is $0.19 \%, 0.00 \%$ and $0.04 \%$, respectively. None of these numbers are statistically significant.

${ }^{3}$ As Figure 9 in Appendix C shows, professional forecasters in that quarter were predicting one-year ahead growth of $2.42 \%$, while the constructed household forecast is $-1.95 \%$.
} 

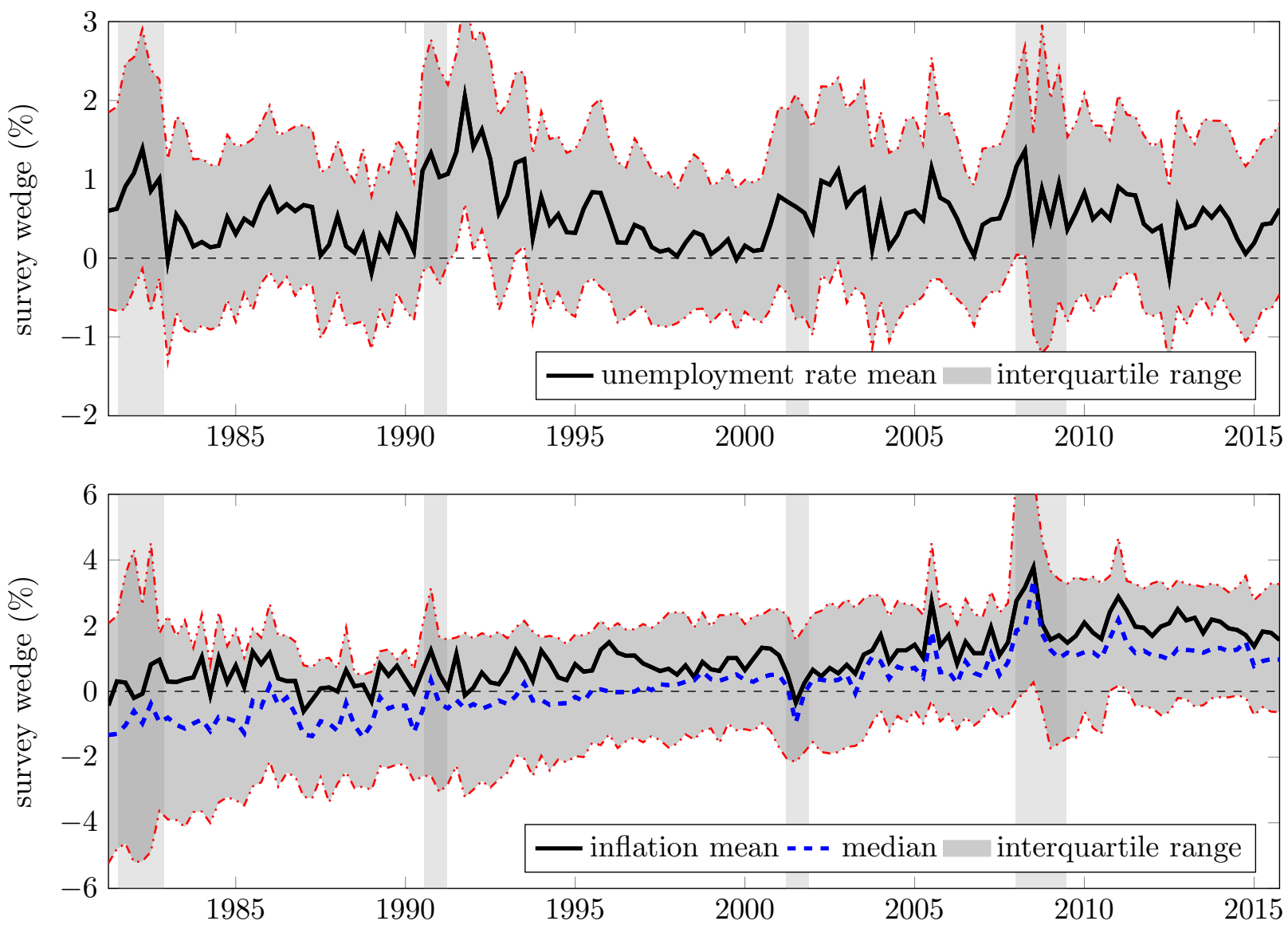

Figure 2: Dispersion in survey expectations in the Michigan Survey. The graphs show different quantiles of the distribution of responses in the Michigan survey, net of the mean response in the Survey of Professional Forecasters. The top panel shows the unemployment responses, bottom panel the inflation responses. Details on the construction of the data series are in Appendix C. NBER recessions shaded.

and therefore implies more ambiguity. Ilut and Schneider (2014) apply the same logic to use the dispersion in the SPF forecasts as a proxy of household confidence in the forecasting model.

We plot the dispersion data from the Michigan survey for the unemployment rate and inflation rate forecasts in Figure 2 for comparison. For the inflation data, we have information on the quantiles of the cross-sectional distribution. For the unemployment rate forecast, we fit a sequence of normal distributions to categorical answers using the same method as in Carlson and Parkin (1975) and Mankiw et al. (2003), see Appendix C for details.

There is indeed substantial cross-sectional dispersion in the survey answers across individual households. However, the interquartile range appears to be stable (except for the inflation answers from early 1980s), and in the case of the unemployment answer also visibly comoves with the business cycle. In Table 6 in Appendix C, we also report average inflation and unemployment forecasts from the Michigan Survey for alternative age groups, geographical regions, quartiles of 
the income distribution, men and women, and different levels of education. While there is some heterogeneity in the biases, usually in the expected direction, substantial biases persist even for groups with high incomes and high levels of acquired education. This justifies our assumption of systematic pessimistic biases in the broad population of households.

While it may be appealing to use cross-sectional dispersion in forecasts as a proxy for the ambiguity concerns of each individual household, our theory does not provide such a direct link. We seek to keep ambiguity concerns separate from the notion disagreement in forecasts across households. The model we develop in this paper is based on a representative agent framework that does not feature heterogeneity in individual forecasts, and therefore yields no predictions about forecast dispersion measures. However, it is possible to extend the framework by introducing heterogeneity in agents' concerns for uncertainty. Agents with differing degrees of ambiguity aversion deduce alternative worst-case models from observable data, which then generates dispersion in forecasts in the model. While conceptually interesting, this extension is beyond the scope of this paper.

\subsection{News shocks and learning}

Fluctuations in macroeconomic survey forecasts have also been investigated through the lens of other theories. Barsky and Sims (2012) study the impact of innovations to the measure of consumer confidence from the Michigan Survey and decompose these innovations into the contribution of news shocks, representing arrival of information about future productivity (Pigou (1927), Beaudry and Portier (2004)), and 'animal spirits' that capture fluctuations in agents' subjective beliefs. We address the decomposition problem by constructing the belief wedge as the difference between households' and professionals' forecasts, thus differencing out the impact of news shocks while preserving the role of fluctuations in subjective beliefs in the form of the households' worst-case model.

Carroll (2003), Reis (2009), Coibion and Gorodnichenko (2012) and many others contribute to the large literature on learning and information acquisition in macroeconomics, imposing alternative learning mechanisms on the side of economic agents. Learning is a plausible way of introducing a wedge between agents' beliefs and the data-generating measure, but it does not explain the large and systematic pessimistic biases observed in household survey responses. Further, it is generally inconsistent with the cross-equation restrictions in the structural model that we derive in Section 4, which imply larger biases for shocks with a more adverse utility impact. Finally, learning models imply slow adjustment of agents' beliefs to economic shocks, and would therefore predict a relatively optimistic bias in recessions, as agents do not fully incorporate the adverse realization of the current state. We observe the opposite correlation between belief wedges and the business cycle in the data. We consider a combination of ambiguity concerns and learning to be an appealing extension, see Epstein and Schneider (2007), Hansen and Sargent (2007, 2010) or Bhandari (2015), but as in the case of belief heterogeneity leave it for further research. 


\section{A one-factor model of distorted beliefs}

We want to formalize the empirical facts that we established in the previous section. In order to do that, we specify a statistical model that describes the joint dynamics of macroeconomic variables and households' expectations. In this model, households' expectations are allowed to differ from the expectations implied by the distribution of the data-generating process in ways consistent with the robust preference specification derived in Section 4.

We extract a common component in the variation of the belief wedge data, and study its impact on the dynamics of the macroeconomic variables. We allow this component to depend on a latent factor that represents exogenous movements in households' expectations, relating the resulting statistical setup to the factor-augmented vector autoregression (FAVAR) approach of Ang and Piazzesi (2003) and Bernanke et al. (2005). Formally, this common component is a restricted version of a change of measure that links subjective beliefs and the data-generating process, derived in Appendix A. Piazzesi et al. (2015) and Jurado (2015) use analogous specifications to model subjective beliefs in the bond market and in the macroeconomy, respectively.

We specify a $(k-1) \times 1$ vector of observable economic variables $y_{t}$ and an unobservable scalar latent process $f_{t}$. In particular, consider the model

$$
\begin{aligned}
& y_{t+1}=\psi_{y} y_{t}+\psi_{y f} f_{t+1}+\psi_{y w} w_{t+1}^{y} \\
& f_{t+1}=\rho_{f} f_{t}+\sigma_{f} w_{t+1}^{f}
\end{aligned}
$$

where $w_{t+1}^{\prime}=\left(\left(w_{t+1}^{y}\right)^{\prime}, w_{t+1}^{f}\right) \sim N\left(0, I_{k}\right)$ is a $k \times 1$ vector of normally distributed iid shocks. We can rewrite these equations, expressing the joint process $x_{t} \doteq\left(y_{t}^{\prime}, f_{t}\right)^{\prime}$ as follows:

$$
\left(\begin{array}{c}
y_{t+1} \\
f_{t+1}
\end{array}\right)=\left(\begin{array}{cc}
\psi_{y} & \psi_{y f} \rho_{f} \\
0 & \rho_{f}
\end{array}\right)\left(\begin{array}{c}
y_{t} \\
f_{t}
\end{array}\right)+\left(\begin{array}{cc}
\psi_{y w} & \psi_{y f} \sigma_{f} \\
0 & \sigma_{f}
\end{array}\right)\left(\begin{array}{c}
w_{t+1}^{y} \\
w_{t+1}^{f}
\end{array}\right) .
$$

This process generates a filtered probability space $\left(\Omega,\left\{\mathcal{F}_{t}\right\}_{t=0}^{\infty}, P\right)$ where $P$ is the objective, datagenerating probability measure. Households' expectations are represented by a subjective probability measure $\widetilde{P}$ that can differ from $P$. We describe the construction of $\widetilde{P}$ next.

Let $\zeta_{t}$ be a subset of observable variables $y_{t}$ for which survey data is available. We define the $\tau$-period belief wedge $\Delta_{t}^{(\tau)}$ as the difference between the $\tau$-period forecasts under the beliefs of the households and under objective expectations:

$$
\Delta_{t}^{(\tau)} \doteq \widetilde{E}_{t} \zeta_{t+\tau}-E_{t} \zeta_{t+\tau}
$$

where $\widetilde{E}_{t} \zeta_{t+\tau}$ is the time- $t$ expectation of $\zeta_{t+\tau}$ under the subjective probability measure of the households. In addition we define the $\tau$-period average belief wedge $\bar{\Delta}_{t}^{(\tau)}$ as the average difference 
in forecasts under the beliefs of the households and under objective expectations:

$$
\bar{\Delta}_{t}^{(\tau)} \doteq \frac{1}{\tau} \sum_{s=1}^{\tau} \Delta_{t}^{(s)}
$$

We impose that the dynamics of belief wedges $\Delta_{t}^{(\tau)}$ and $\bar{\Delta}_{t}^{(\tau)}$ can be summarized using the scalar factor

$$
\theta_{t}=\left(F_{y}, F_{f}\right)\left(\begin{array}{c}
y_{t} \\
f_{t}
\end{array}\right) .
$$

Individual one-period forecasts of the innovation means under the households' expectations are then represented by a vector of factor loadings $H$ :

$$
\widetilde{E}_{t}\left[w_{t+1}\right]=H \theta_{t}
$$

Applying the law of iterated expectations, belief wedges for the $\tau$-period forecasts can be written as

$$
\Delta_{t}^{(\tau)}=G_{x}^{(\tau)} x_{t}+G_{0}^{(\tau)}
$$

where the coefficients $G_{x}^{(\tau)}$ and $G_{0}^{(\tau)}$ are derived in Appendix A.

While we specified a flexible VAR specification for the dynamics of observable variables, we imposed tight restrictions on the households' expectations. The model (2)-(3) implies a one-factor structure of belief wedges where $\theta_{t}$ captures the common comovement in these wedges. In this latent factor model, we interpret $\theta_{t}$ as the time-varying measure of pessimism among the households reflected in the survey data that impacts the dynamics of macroeconomic variables. In Section 4 , this one-factor structure together with particular restrictions on $H$ and $F=\left(F_{y}, F_{f}\right)$ is derived from the decision problem of the household endowed with robust preferences, where $\theta_{t}$ reflects the time-variation in households' ambiguity concerns.

\subsection{Data and estimation}

Data on macroeconomic variables are obtained from the Federal Reserve Bank of St. Louis database (FRED), at quarterly frequency. The vector $y_{t}$ includes real GDP growth, the unemployment rate, inflation, and the Federal Funds rate. We include three belief wedges from Figure 1 in the vector $\bar{\Delta}_{t}^{(4)}$, constructed as 4-quarter ahead average belief wedges between the Michigan Survey and SPF forecasts for GDP growth, the unemployment rate and inflation. Appendix C provides details on the construction of the data, presented in Section 2. The data for $y_{t}$ covers the period 1960Q22015Q4. The belief wedges for GDP growth, the unemployment rate and inflation cover the periods 1968Q4-2015Q4, 1977Q4-2015Q4, and 1981Q2-2015Q4 respectively.

In order to keep the estimation and interpretation of the model transparent, we restrict the dynamics of beliefs and set $F_{y}=0$, thereby setting $\theta_{t}=f_{t}$. This implies that fluctuations in the belief wedges are driven purely by the belief factor $f_{t}$, and not directly by the dynamics of 
endogenous macroeconomic variables $y_{t}$. In addition, we normalize $F_{f}=1$ and set the element of $H$ corresponding to the GDP growth shock to be -1 in order to identify the sign and scale of $\theta_{t} .{ }^{4}$

More specifically, we estimate the model (1) together with a vector of observation equations for the wedges

$$
\bar{\Delta}_{t+1}^{(4)}=\psi_{\Delta f} f_{t+1}+\sigma_{\Delta} \varepsilon_{t+1}^{\Delta}
$$

where $\sigma_{\Delta}$ is diagonal and $\varepsilon_{t+1}^{\Delta} \sim N(0, I)$ is a vector of normally distributed iid measurement errors. $y_{t}$ and $\bar{\Delta}_{t}^{(4)}$ are demeaned. We introduce a measurement error for every belief wedge in order to absorb idiosyncratic noise in the survey responses, and focus on the extraction of the persistent common factor predicted by the theoretical model. We seek estimates for the parameters

$$
\left\{\psi_{y}, \psi_{y f}, \psi_{y w}, \rho_{f}, \sigma_{f}, H, \sigma_{\Delta}\right\}
$$

and the belief factor $\theta_{t}=f_{t}$. Appendix A solves for $\psi_{\Delta f}$ from the above parameters.

We estimate the model using Bayesian methods. Further details, including the imposed priors and estimated posteriors are summarized in Appendix D.

\section{$3.2 \quad$ Results}

A variance decomposition at the modal parameter estimate, summarized in Table 8 in Appendix D, reveals that the factor shock explains $67.5 \%, 23.2 \%$, and $9.7 \%$ of the variation in the GDP growth wedge, unemployment wedge, and inflation wedge respectively. These results confirm the strong correlation between the belief wedges that concern real quantities. Moreover, the posterior estimates shown in Table 8 in Appendix D reveal a very tightly identified persistence $\rho_{f}$ of this factor with posterior mean of 0.8 at the quarterly frequency. The fact that a sizeable fraction of variation in the wedges is explained by the persistent fluctuations in the factor $\theta_{t}$ provides evidence of systematic comovement in households' beliefs about future economic outcomes.

Figure 3 plots the impulse response functions of $y_{t}$ and $\bar{\Delta}_{t}^{(4)}$ to a positive one standard deviation shock $w_{t}^{f}$ to $\theta_{t}=f_{t}$, with the factor response plotted in the bottom right panel. An increase in $\theta_{t}$ leads household forecasts for GDP growth to be biased further downward relative to the SPF forecasts, while the biases in the household forecasts for unemployment and inflation increase relative to the SPF forecasts. The impulse responses of the belief wedges are consistent with the correlations and average signs of the wedges described in Section 2.

These results support the interpretation of $\theta_{t}$ as a time-varying measure of the level of pessimism among households. From the perspective of the robust preference model that we develop in the next section, households are concerned about a future path that exhibits low GDP growth, a high unemployment rate and high inflation. An increase in $\theta_{t}$ makes these concerns stronger, biasing households' beliefs more strongly in this direction.

\footnotetext{
${ }^{4}$ The shock exposure matrix $\psi_{y w}$ is only identified as the covariance matrix $\psi_{y w} \psi_{y w}^{\prime}$. For the purpose of estimation, we shall impose a recursive identification scheme for $\psi_{y w}$. However, $\psi_{y w}$ only appears as $\psi_{y w} \psi_{y w}^{\prime}$ in the formulas for the belief wedges. Therefore, given an estimate of $\psi_{y w} \psi_{y w}^{\prime}$, the identification of $\psi_{y w}$ does not play a role in the estimation of the factor shocks $w_{t}^{f}$.
} 

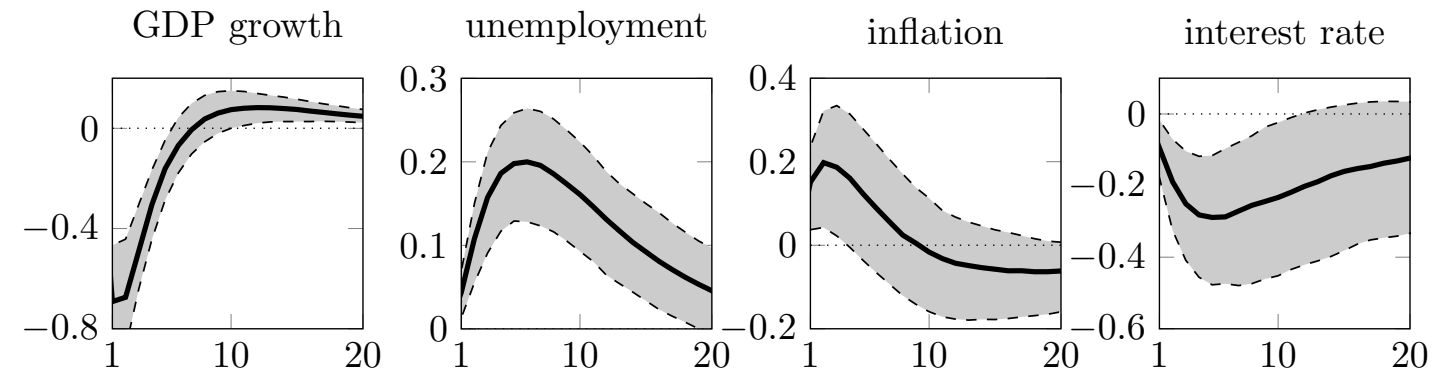

GDP growth wedge unemployment wedge inflation wedge
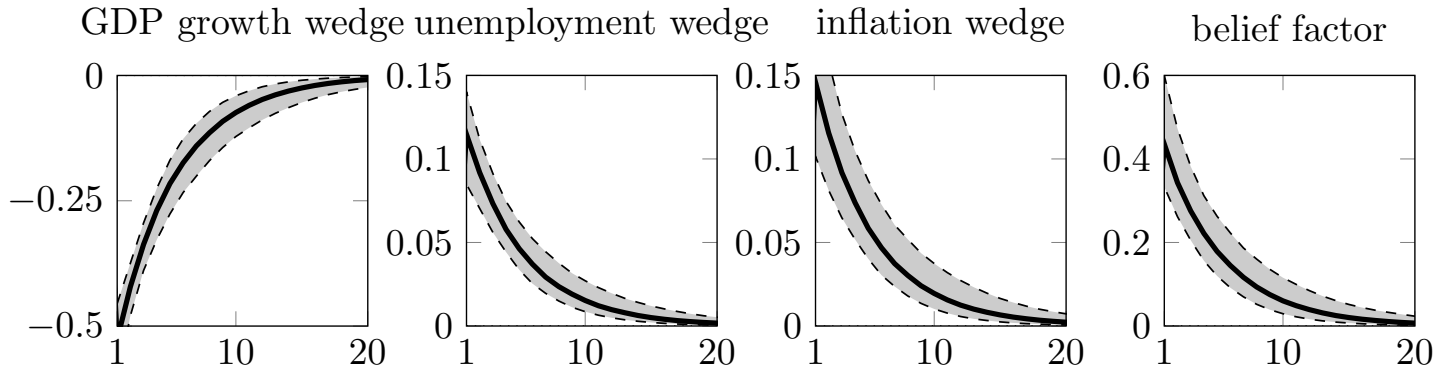

Figure 3: Bayesian impulse response functions to the belief shock $w^{f}$ in the factor model. The solid lines indicate median estimates, while the dashed line correspond to 10th and 90th percentile error bands. GDP growth, inflation, and interest rate are annualized and in percentage deviations. The unemployment rate is in percentage points. The GDP growth wedge and inflation wedge are scaled to correspond to the belief wedges of annualized GDP growth and annualized inflation. The horizontal axis is in quarters.

The belief shock $w^{f}$ also has real effects. In response to a positive shock to $\theta_{t}$, GDP growth falls and unemployment rises. The impulse response for inflation is positive for the first year and close to zero subsequently. The interest rate declines in response to the belief shock. At the modal parameter estimate, $\theta_{t}$ explains $13.1 \%, 21.0 \%, 4.0 \%$ and $5.3 \%$ of the movements in GDP growth, unemployment, inflation and interest rates, respectively.

Our estimates suggest that a rise in pessimism has contractionary effects, and we emphasize the especially large adverse response of unemployment. In Section 5, we develop and estimate a structural macroeconomic model with a frictional labor market and ambiguity averse agents and revisit these empirical findings. In line with the results from the factor model, the ambiguity shock in the structural model generates nontrivial recessionary responses, with a particularly pronounced response in the labor market.

In Appendix G, we conduct two robustness exercises. First, we reestimate the factor model using the median household inflation forecast instead of the mean and show that the results remain virtually unchanged. Second, we replace the SPF forecast with the model-implied rational forecast in the construction of the belief wedges. In this case, we find quantitatively modest differences that we interpret as supportive of our approach in the construction of the belief wedges. 


\section{Robust preferences}

Motivated by the empirical results from Sections 2 and 3, we now introduce a preference specification that generates endogenous deviations of agents' beliefs from the data-generating probability measure. This model extends the robust preference framework of Hansen and Sargent (2001a,b) to allow for a more flexible form of belief distortions, similar to Hansen and Sargent (2015). The flexibility allows for time-variation in the degree of agents' pessimism over time, which we identify from survey data, while tightly restricting the structure of pessimistic distortions across individual states, linking them to agents' continuation values and equilibrium dynamics. We then develop an approximation technique that incorporates the effects of time-varying belief distortions in a tractable linear solution.

Agents' preferences are represented using the continuation value recursion

$$
V_{t}=\min _{\substack{m_{t+1}>0 \\ E_{t}\left[m_{t+1}\right]=1}} u\left(x_{t}\right)+\beta E_{t}\left[m_{t+1} V_{t+1}\right]+\frac{\beta}{\theta_{t}} E_{t}\left[m_{t+1} \log m_{t+1}\right]
$$

with period utility $u\left(x_{t}\right)$. Here, $x_{t}$ is an $n \times 1$ vector of stationary economic variables that follows the Markovian law of motion

$$
x_{t+1}=\psi\left(x_{t}, w_{t+1}\right) \text {, }
$$

where $w_{t+1} \sim N\left(0_{k}, I_{k \times k}\right)$ an iid vector of normally distributed shocks under the data-generating probability measure $P .{ }^{5}$

These preferences have been formulated by Hansen and Sargent (2001a,b) as a way of introducing concerns for model misspecification on the side of the agents. The agent treats the measure $P$ as an approximating or benchmark model and considers potential stochastic deviations from this model, represented by the strictly positive, mean-one random variable $m_{t+1}$. The minimization problem in (4) captures the search for a 'worst-case' model that serves as a basis for the agent's decisions. The models that are considered by the agent are difficult to distinguish statistically from the benchmark model, and the degree of statistical similarity is controlled by the entropy penalty $E_{t}\left[m_{t+1} \log m_{t+1}\right]$, scaled by the penalty parameter $\theta_{t}$. More pronounced statistical deviations that are easier to detect are represented by random variables $m_{t+1}$ with a large dispersion that yields a large entropy. Here, recursion (4) is specified for a fixed stochastic utility flow $u\left(x_{t}\right)$. In Section 5 , we endow the agent with a set of controls, which gives rise to a min-max specification of the recursion.

The preferences considered by Hansen and Sargent (2001a,b) impose a constant parameter $\theta>0$. As $\theta \searrow 0$, the penalty for deviating from the benchmark model becomes more severe, and the resulting preferences approach a utility-maximizing agent with rational expectations.

We are interested in a specification that permits more flexibility in the set of models that the

\footnotetext{
${ }^{5}$ For now we take the function $\psi$ as given, but later derive it as a solution to a set of equilibrium conditions.
} 
agent views as plausible. In particular, we envision the time-varying model

$$
\theta_{t}=\bar{\theta} x_{t}
$$

where $\bar{\theta}$ is a $1 \times n$ vector of parameters. The solution of (4) satisfies

$$
m_{t+1}=\frac{\exp \left(-\theta_{t} V_{t+1}\right)}{E_{t}\left[\exp \left(-\theta_{t} V_{t+1}\right)\right]}
$$

and $m_{t+1}$ completely characterizes the worst-case model distortions relative to the benchmark model. The variation in $\theta_{t}$ thus implies a time-varying model for the worst-case distortion. ${ }^{6}$ The chained sequence of random variables $m_{t+1}$ specifies a strictly positive martingale $M$ recursively as $M_{t+1}=m_{t+1} M_{t}$ with $M_{0}=1$ that defines a probability measure $\widetilde{P}$ with conditional expectations

$$
\widetilde{E}_{t}\left[x_{t+1}\right] \doteq E_{t}\left[m_{t+1} x_{t+1}\right]
$$

Consequently, the wedge between the one-period forecasts of $x_{t+1}$ under the worst-case and benchmark models is given by

$$
\Delta_{t} \doteq \widetilde{E}_{t}\left[x_{t+1}\right]-E_{t}\left[x_{t+1}\right]
$$

Notice that the distortion (7) implies a large value of $m_{t+1}$ for low realizations of the continuation value $V_{t+1}$. The worst-case model, represented by the probability measure $\widetilde{P}$, thus overweighs adverse states as ranked by the preferences of the agent. In this way, the preference model implies tightly restricted endogenous pessimism on the side of the agents, generated by concerns for model misspecification. The degree of pessimism is controlled by the evolution of $\theta_{t}$.

\subsection{A linear approximation}

A wide range of dynamic stochastic general equilibrium models with robust agents can be cast as a solution to a system of expectational difference equations:

$$
0=E_{t}\left[\widetilde{g}\left(x_{t+1}, x_{t}, x_{t-1}, w_{t+1}, w_{t}\right)\right]
$$

where $\widetilde{g}$ is an $n \times 1$ vector function. This vector of equations includes Euler equations of the robust household, which can be represented using worst-case belief distortions $m_{t+1}$ that are embedded in $\widetilde{g}$. We are interested in deriving a tractable approximation of the equilibrium dynamics for $x_{t}$ in the form of a Markovian law of motion of the form (5) from the system of equations (9). Part of the solution is the worst-case distortion (7) with continuation values $V_{t+1}$ consistent with the equilibrium dynamics, and the resulting biases (8). In this section, we sketch out how to compute approximations to equilibria of this class of problems, with detailed calculations with provided in

\footnotetext{
${ }^{6}$ Since $\theta_{t}$ is measurable with respect to the agent's information set at time $t$, the preferences are dynamically consistent. The linear specification of $\theta_{t}$ in general allows for negative values, in which case the conditional minimization problem in (4) turns into a maximization problem of an 'ambiguity-loving' agent, and the distortion (7) implies optimistic biases in survey responses.
} 
Appendix B.

Our approximation is constructed using a perturbation technique in the spirit of Sims (2002) and Schmitt-Grohé and Uribe (2004). Intuitively, the method builds a small-noise expansion around agents' worst-case model, in order to deliver time-varying effects of ambiguity concerns in a linear approximation of the solution. The challenge stems from the endogeneity of the worst-case model that agents use as a basis for their decisions. The feedback between agents' worst-case model and the equilibrium law of motion requires jointly solving for the continuation value recursion (4), the probability measure $\widetilde{P}$, and the law of motion (5).

Assuming that the function $\psi(x, w)$ is sufficiently smooth, we combine the series expansion method of Holmes (1995) and Lombardo (2010) with an extension of the worst-case model approximation used in Borovička and Hansen (2013, 2014). The method, outlined in detail in Appendix B, approximates the dynamics in the neighborhood of the deterministic steady state $\bar{x}$ that is given by the solution to $\bar{x}=\psi(\bar{x}, 0)$. The dynamics of the state vector $x_{t}$ can be approximated as

$$
x_{t} \approx \bar{x}+\mathrm{q} x_{1 t}
$$

where $\mathrm{q}$ is a perturbation parameter. The law of motion for the 'first-derivative' process $x_{1 t}$ that represents the local dynamics in the neighborhood of the steady state can be derived from the linear approximation of (5):

$$
x_{1 t+1}=\psi_{q}+\psi_{x} x_{1 t}+\psi_{w} w_{t+1}
$$

where $\psi_{q}, \psi_{x}$ and $\psi_{w}$ are conforming coefficient matrices. Similarly, we can construct a linear approximation of the continuation value (4) where the deviation of the continuation value from its steady state satisfies

$$
V_{1 t}=V_{x} x_{1 t}+V_{q}
$$

We show in Appendix B how to construct the solution (10)-(11) from a set of equilibrium conditions (9), and how the solution depends on the dynamics of $\theta_{t}$. Further, we show that under the agent's worst-case model $\widetilde{P}$, the innovations $w_{t+1}$ are distributed as

$$
w_{t+1} \sim N\left(-\bar{\theta}\left(\bar{x}+x_{1 t}\right)\left(V_{x} \psi_{w}\right)^{\prime}, I_{k \times k}\right)
$$

Instead of facing a vector of zero-mean shocks $w_{t+1}$, the agent perceives these shocks as having a time-varying drift. The time-variation is determined by a linear approximation to $\theta_{t}$ from equation (6), given by $\bar{\theta}\left(\bar{x}+x_{1 t}\right)$. The relative magnitudes of the distortions of individual shocks are given by the sensitivity of the continuation value to the dynamics of the state vector, $V_{x}$, and the loadings of the state vector on individual shocks, $\psi_{w}$. The agent perceives larger distortions during periods when $\theta_{t}$ is large, and distorts relatively more the shocks which impact the continuation value more strongly. 
Consequently, the dynamics of the model (10) under the agents' worst-case beliefs satisfy

$$
\begin{aligned}
x_{1 t+1} & =\left[\psi_{q}-\psi_{w} \psi_{w}^{\prime} V_{x}^{\prime} \bar{\theta} \bar{x}\right]+\left[\psi_{x}-\psi_{w} \psi_{w}^{\prime} V_{x}^{\prime} \bar{\theta}\right] x_{1 t}+\psi_{w} \widetilde{w}_{t+1} \\
& =\widetilde{\psi}_{q}+\widetilde{\psi}_{x} x_{1 t}+\psi_{w} \widetilde{w}_{t+1} .
\end{aligned}
$$

The worst-case model alters both the conditional mean and the persistence of economic shocks. Moreover, variables that tend to move ambiguity and the continuation value in opposite directions tend to exhibit a higher persistence under the worst-case model. ${ }^{7}$

\subsection{Worst-case model and survey responses}

In Section 3, we estimated a one-factor model of biases embedded in survey responses on household expectations of future economic variables. The preference framework introduced in this section derives these biases using the endogenous worst-case probability distribution $\widetilde{P}$. Assuming that

surveyed households provide answers regarding economic forecasts using $\widetilde{P}$ we can connect the empirical observations on survey responses to the theoretical predictions on decisions under robust preferences.

Using the survey data and the rational forecasts from the linearized model (10), we identify the belief wedges (8) as

$$
\Delta_{t}^{(1)}=\psi_{w} \widetilde{E}_{t}\left[w_{t+1}\right]=-\bar{\theta}\left(\bar{x}+x_{1 t}\right)\left(\psi_{w} \psi_{w}^{\prime}\right) V_{x}^{\prime}
$$

The one-factor structure in survey answers is driven by the time-variation in $\bar{\theta}\left(\bar{x}+x_{1 t}\right)$, with the constant vector of loadings $-\left(\psi_{w} \psi_{w}^{\prime}\right) V_{x}^{\prime}$. This is the key restriction that the robust preference model imposes on the joint dynamics of the survey answers.

Observe that this specification of belief wedges is a restricted case of the reduced-form model (1)-(3). In the notation from Appendix A, we have

$$
F=\bar{\theta}, \quad H=-\left(\psi_{w} \psi_{w}^{\prime}\right) V_{x}^{\prime}, \quad \bar{H}=-\bar{\theta} \bar{x}\left(\psi_{w} \psi_{w}^{\prime}\right) V_{x}^{\prime}
$$

The terms $\bar{\theta}, \psi_{w}, V_{x}$ are functions of structural parameters in the model. Belief wedges for longerhorizon forecasts are then computed using formulas from Appendix A.

\subsection{Dealing with non-stationarities}

For the purpose of applying the expansion method, we assumed that the state vector $x_{t}$ is stationary. Our framework can, however, deal with deterministic or stochastic trends featured in macroeconomic models. Specifically, let us assume that there exists a vector-valued stochastic process $z_{t}$ such that the dynamics of $x_{t}$ can be written as

$$
\begin{aligned}
x_{t} & =\widehat{x}_{t}+z_{t} \\
z_{t+1}-z_{t} & =\phi\left(\widehat{x}_{t}, w_{t+1}\right)
\end{aligned}
$$

\footnotetext{
${ }^{7}$ This statement is precisely correct in the scalar case, when $\psi_{x}^{2} V_{x} \bar{\theta}<0$.
} 
where $\widehat{x}_{t}$ is a stationary vector Markov process that replaces dynamics (5):

$$
\widehat{x}_{t+1}=\psi\left(\widehat{x}_{t}, w_{t+1}\right)
$$

The process $z_{t}$ thus has stationary increments and $x_{t}$ and $z_{t}$ are cointegrated, element by element. A typical example of an element in $z_{t}$ is a productivity process with a permanent component. Once we solve for the stationary dynamics of $\widehat{x}_{t}$, we can obtain the dynamics of $x_{t}$ in a straightforward way using (14).

In order to compute the non-stationary version of the continuation value recursion and the appropriate worst-case distortions, consider as an example

$$
u\left(x_{t}\right)=\log C_{t}=\log \left[\widehat{C}_{t} \exp \left(z_{t}\right)\right]=\log \widehat{C}_{t}+z_{t},
$$

where $C_{t}$ is agent's consumption process and $\widehat{C}_{t}=\widehat{C}\left(\widehat{x}_{t}\right)$ is its stationary rescaling. We show in Appendix B.6 that in this case, the continuation value can be written as

$$
V_{t}=\widehat{V}\left(\widehat{x}_{t}\right)+\frac{1}{1-\beta} z_{t}
$$

and the worst-case model distortion is given by

$$
m_{t+1}=\frac{\exp \left(-\theta_{t}\left(\widehat{V}\left(\widehat{x}_{t+1}\right)+(1-\beta)^{-1} \phi\left(\widehat{x}_{t}, w_{t+1}\right)\right)\right)}{E_{t}\left[\exp \left(-\theta_{t}\left(\widehat{V}\left(\widehat{x}_{t+1}\right)+(1-\beta)^{-1} \phi\left(\widehat{x}_{t}, w_{t+1}\right)\right)\right)\right]} .
$$

This type of belief distortion has stationary increments $m_{t+1}$ and can be dealt with by applying the first-order series expansion to the functions $\widehat{V}\left(\widehat{x}_{t+1}\right)$ and $\phi\left(\widehat{x}_{t}, w_{t+1}\right)$ as above. Consequently, the worst-case distribution of the shock vector is given by

$$
w_{t+1} \sim N\left(-\bar{\theta}\left(\bar{x}+\widehat{x}_{1 t}\right)\left(V_{x} \psi_{w}+(1-\beta)^{-1} \phi_{w}\right)^{\prime}, I_{k \times k}\right) .
$$

The distortions thus inherit the contribution of the increment $(1-\beta)^{-1} \phi_{w}$ of the non-stationary process $z_{t}$ to the dynamics of the continuation value. The worst-case dynamics (12) and the belief wedges (13) are modified accordingly. Specifically, we can compute the multiperiod belief wedges $\Delta_{t}^{(\tau)}$ using the recursive calculations outlined in Appendix A, imposing

$$
\begin{aligned}
F & =\bar{\theta} \\
H & =-\psi_{w}\left(V_{x} \psi_{w}+(1-\beta)^{-1} \phi_{w}\right)^{\prime} \\
\bar{H} & =-(\bar{\theta} \bar{x}) \psi_{w}\left(V_{x} \psi_{w}+(1-\beta)^{-1} \phi_{w}\right)^{\prime}
\end{aligned}
$$




\section{$5 \quad$ A structural business cycle model}

In this section we introduce the robust preference framework from Section 4 into a dynamic stochastic general equilibrium model of the macroeconomy. We use this model to shed light on the observations in Section 3, especially the role of ambiguity shocks in explaining large fluctuations in labor market outcomes.

We implement and estimate a version of the New-Keynesian framework with a frictional labor market introduced in Ravenna and Walsh (2008) and Christiano et al. (2015). In the frictional labor market with search and matching, incentives of workers and firms to search for jobs and post job vacancies are directly linked to their forecasts about the present value of a potential match. Ambiguity shocks impact this present value by overweighting the probability of states with low continuation values for the households, which are correlated with low values of the worker-firm matches. Moreover, the search and matching environment and nominal rigidities provide a welldefined notion of unemployment and inflation which directly map to the survey questions.

\subsection{Model}

The model economy is populated by a representative household endowed with robust preferences, competitive producers of a homogeneous final good, and a two-tier structure of monopolistic producers of intermediate goods who hire workers in a frictional labor market. Here, we focus on key components of the model relevant for the analysis of the role of ambiguity shocks — households' preferences, contracting in the labor market, and specification of exogenous sources of variation in the model - deferring additional details to Appendix E. ${ }^{8}$

\subsubsection{Representative household}

The preferences of the representative household are given by the recursion

$$
V_{t}=\min _{\substack{m_{t+1}>0 \\ E_{t}\left[m_{t+1}\right]=1}} \max _{C_{t}, I_{t}, B_{t+1}} u\left(x_{t}\right)+\beta E_{t}\left[m_{t+1} V_{t+1}\right]+\frac{\beta}{\theta_{t}} E_{t}\left[m_{t+1} \log m_{t+1}\right]
$$

with time preference coefficient $\beta$ and period utility over aggregate consumption $C_{t}$,

$$
u\left(x_{t}\right)=\log \left(C_{t}-b C_{t-1}\right)
$$

where $b$ determines the degree of habit formation. In line with our factor model specification from Section 3 , we assume that the stochastic process for the robust concerns is given by $\theta_{t}=\bar{\theta} x_{t} \doteq f_{t}$ where $f_{t}$ follows an $\operatorname{AR}(1)$ process

$$
f_{t+1}=\left(1-\rho_{f}\right) \bar{f}+\rho_{f} f_{t}+\sigma_{f} w_{t+1}^{f} .
$$

\footnotetext{
${ }^{8} \mathrm{~A}$ concise set of model equations can be found in the online technical appendix to Christiano et al. (2015).
} 
The worst-case belief of the household is

$$
m_{t+1}=\frac{\exp \left(-\theta_{t} V_{t+1}\right)}{E_{t}\left[\exp \left(-\theta_{t} V_{t+1}\right)\right]}
$$

The magnitude of the belief distortion is determined by fluctuations in $\theta_{t}$ specified exogenously in (18). However, the equilibrium dynamics in the model endogenously determines which states yield low continuation values $V_{t+1}$ and are therefore evaluated as adverse by the household. These states are then perceived as more likely under the worst-case model. Naturally, the dynamics of the worst-case belief then endogenously depends on other sources of shocks introduced into the model, which we describe in Section 5.1.4.

The household faces the budget constraint

$$
P_{t} C_{t}+P_{I, t} I_{t}+B_{t+1} \leq\left(R_{K, t} u_{t}^{K}-a_{u}\left(u_{t}^{K}\right) P_{I, t}\right) K_{t}+\left(1-l_{t}\right) P_{t} D_{t}+\xi_{t} l_{t}+R_{t-1} B_{t}-T_{t}
$$

$P_{t}$ is the price of consumption goods and $P_{I, t}$ is the price of investment goods. $B_{t+1}$ denotes the one-period risk-free bonds purchased in period $t$ with return $R_{t}, I_{t}$ is the quantity of investment goods and $T_{t}$ lump sum taxes net of profits. Household's capital stock $K_{t}$ earns rental rate $R_{K, t}$, is utilized at rate $u_{t}^{K}$ subject to capital utilization cost $a_{u}\left(u_{t}^{K}\right)$, and follows the law of motion

$$
K_{t+1}=\left(1-\delta_{K}\right) K_{t}+\left(1-a_{I}\left(\frac{I_{t}}{I_{t-1}}\right)\right) I_{t}
$$

where $a_{I}(\cdot)$ is an adjustment cost that is increasing and convex.

\subsubsection{Labor market}

The household consists of a unit mass of workers who perfectly share consumption risk. Fraction $l_{t}$ is employed and earns a wage $\xi_{t}$. Fraction $1-l_{t}$ is unemployed and collect unemployment benefits $D_{t}$ financed through lump sum taxes. At the end of period $t$, employed workers separate with probability $1-\rho$ and join the pool of unemployed who search for jobs at the beginning of period $t+1$. The total number of searchers at the beginning of period $t+1$ therefore is $1-\rho l_{t}$ and these searchers face a job finding probability $j_{t+1}$. The law of motion for the mass of employed workers thus is

$$
l_{t+1}=\rho l_{t}+\left(1-\rho l_{t}\right) j_{t+1}=\left(\rho+\eta_{t+1}\right) l_{t}
$$

where

$$
\eta_{t+1}=\frac{j_{t+1}\left(1-\rho l_{t}\right)}{l_{t}}
$$

is the hiring rate. The value of an employed worker is

$$
W_{t}=\xi_{t}+\widetilde{E}_{t}\left[\frac{S_{t+1}}{S_{t}}\left(\left(\rho+(1-\rho) j_{t+1}\right) W_{t+1}+(1-\rho)\left(1-j_{t+1}\right) U_{t+1}\right)\right]
$$


where $S_{t+1} / S_{t}$ is the period $t$ stochastic discount factor, $\xi_{t}$ is the period $t$ wage, and $U_{t+1}$ is the value of being unemployed next period, given by the recursion

$$
U_{t}=D_{t}+\widetilde{E}_{t}\left[\frac{S_{t+1}}{S_{t}}\left(j_{t+1} W_{t+1}+\left(1-j_{t+1}\right) U_{t+1}\right)\right] .
$$

Denote by $\vartheta_{t}$ the real marginal revenue in period $t$ from hiring an additional worker. The value of the worker to a firm is given by the revenue generated in the match net of the wages paid,

$$
J_{t}=\vartheta_{t}-\xi_{t}+\rho \widetilde{E}_{t}\left[\frac{S_{t+1}}{S_{t}} J_{t+1}\right] .
$$

Free entry of firms implies that in equilibrium,

$$
Q_{t}\left(J_{t}-\kappa_{t}\right)=s_{t}
$$

where $Q_{t}$ is the probability of filling a vacancy, $\kappa_{t}$ is the fixed cost of hiring a worker, and $s_{t}$ is the cost of posting a vacancy.

The important insight in this frictional labor market is that expectations operators in recursions (20)-(21) inherit the probability measure $\widetilde{P}$, indicating that both workers and firms evaluate the distribution of future values of $W_{t}, U_{t}$ and $J_{t}$ under the worst-case beliefs of the household. Ambiguity shocks then directly affect the incentives of firms to hire through their effect on the valuation of the match surplus.

This is a striking difference relative to the Walrasian spot market where workers are hired only using one-period employment contracts. In such an environment, ambiguity concerns are absent from the labor market decisions, since there is no uncertainty about economic conditions prevailing in the given period.

What remains to be determined is the split of the surplus from a match between the firm's surplus, $J_{t}$, and the worker's surplus, $W_{t}-U_{t}$. As in Hall and Milgrom (2008) and Christiano et al. (2015), we adopt the alternating offer bargaining protocol of Rubinstein (1982) and Binmore et al. (1986). The outcome of this bargaining mechanism is the surplus splitting rule

$$
J_{t}=\beta_{1}\left(W_{t}-U_{t}\right)-\beta_{2} \gamma_{t}+\beta_{3}\left(\vartheta_{t}-D_{t}\right)
$$

with parameters $\beta_{i}, i=1,2,3$ that depend on the parameters of the bargaining problem and are described in detail in Appendix E. Notice that when $\beta_{2}=\beta_{3}=0$, we obtain the Nash bargaining solution with worker's share $\left(1+\beta_{1}\right)^{-1}$. Relative to the Nash bargaining solution, the alternative offer bargaining makes the firm's surplus more procyclical, leading to smoother wages and more procyclical hiring patterns over the business cycle. 


\subsubsection{Production and market clearing}

The frictional labor market is embedded in a New-Keynesian framework with Calvo (1983) price setting. A homogeneous final good $Y_{t}$ with price $P_{t}$ is produced in a competitive market using the production technology

$$
Y_{t}=\left[\int_{0}^{1}\left(Y_{i, t}\right)^{\frac{1}{\lambda}} d i\right]^{\lambda}, \quad \lambda>1 .
$$

where $Y_{i, t}$ are specialized inputs with prices $P_{i, t}$. Final good producers solve the static competitive problem

$$
\max _{Y_{i, t}} P_{t} Y_{t}-\int_{0}^{1} P_{i, t} Y_{i, t} d i
$$

leading to the first-order conditions

$$
Y_{i, t}=\left(\frac{P_{t}}{P_{i, t}}\right)^{\frac{\lambda}{\lambda-1}} Y_{t}, \quad i \in[0,1] .
$$

Specialized inputs are produced by monopolist retailers indexed by $i$, using the production technology

$$
Y_{i, t}=k_{i, t}^{\alpha}\left(A_{t} h_{i, t}\right)^{1-\alpha}-\phi_{t},
$$

where $k_{i, t}$ is the quantity of capital purchased, $h_{i, t}$ is the quantity of intermediate goods, $A_{t}$ is the neutral technology level, and $\phi_{t}$ is a fixed cost of production. The retailer purchases intermediate goods at price $P_{t}^{h}$ from a wholesaler in a competitive market and must finance the purchase by borrowing $P_{t}^{h} h_{i, t}$ at the nominal interest rate $R_{t}$. The loan is repaid at the end of period $t$ after the retailer receives its sales revenues. Finally, the retailer is subject to the sticky price friction, implying that every period he is allowed to reset the price with probability $1-\chi$.

Intermediate goods are produced by wholesalers using a technology that turns one unit of labor into one unit of intermediate good. This implies the market clearing condition

$$
\int_{0}^{1} h_{i, t} d i=h_{t}=l_{t}
$$

Market clearing for services of capital requires

$$
\int_{0}^{1} k_{i, t} d i=u_{t}^{K} K_{t}
$$

The model is closed with an aggregate resource constraint

$$
C_{t}+\left(I_{t}+a_{u}\left(u_{t}^{K}\right) k_{t}\right) / \Psi_{t}+\left(s_{t} / Q_{t}+\kappa_{t}\right) \eta_{t} l_{t-1}+G_{t}=Y_{t}
$$

where $G_{t}$ denotes government consumption and $\Psi_{t}=P_{t} / P_{I, t}$ denotes the relative price of investment and reflects investment-specific technological progress. 


\subsubsection{Shock structure and monetary policy}

We complete the model by specifying the sources of exogenous variation to the model. The monetary authority follows the interest rate policy rule

$$
\log \left(R_{t} / \bar{R}\right)=\rho_{R} \ln \left(R_{t-1} / \bar{R}\right)+\left(1-\rho_{R}\right)\left[r_{\pi} \log \left(\pi_{t} / \bar{\pi}\right)+r_{y} \log \left(\mathcal{Y}_{t} / \mathcal{Y}_{t}^{*}\right)\right]+\sigma_{R} w_{t}^{R}
$$

where $w_{t}^{R}$ is a monetary policy shock and

$$
\mathcal{Y}_{t}=C_{t}+I_{t} / \Psi_{t}+G_{t}
$$

denotes real GDP. $\mathcal{Y}_{t}^{*}$ is the value of $\mathcal{Y}_{t}$ along the non-stochastic steady state growth path, scaled by the current level of productivity.

Finally, we prescribe the dynamics of technology shocks. The neutral technology process $A_{t}$ exhibits iid growth

$$
\log \left(A_{t} / A_{t-1}\right) \doteq \log \left(\mu_{A, t}\right)=\sigma_{A} w_{t}^{A}
$$

while the investment-specific technological process $\Psi_{t}$ has a mean-reverting growth rate

$$
\log \left(\Psi_{t} / \Psi_{t-1}\right) \doteq \log \left(\mu_{\Psi, t}\right)=\rho_{\Psi} \log \left(\mu_{\Psi, t-1}\right)+\sigma_{\Psi} w_{t}^{\Psi}
$$

The final source of exogenous variation is the ambiguity shock process (18). We assume that all innovations are independent under the data-generating measure $P$ :

$$
\left(w_{t}^{R}, w_{t}^{A}, w_{t}^{\Psi}, w_{t}^{f}\right)^{\prime} \stackrel{i i d}{\sim} N(0, I)
$$

As we have seen in Section 4, this property does not carry over to the worst-case model where the distribution of future realizations of the shocks depends on the current level of ambiguity concern $\theta_{t}$.

Lastly, to ensure a balanced growth path in the non-stochastic steady state, the parameters $\left\{\phi_{t}, s_{t}, \kappa_{t}, \gamma_{t}, G_{t}, D_{t}\right\}$ need to grow at the growth rate of the economy. The details of these adjustments are in Appendix E.2.

\subsection{Model solution and estimation}

The equilibrium of the structural model sketched out in the previous section fits in the general framework that we developed in Section 4. We use the expansion methods from Section 4.1 to compute a linear approximation to the solution for the equilibrium dynamics. This facilitates the implementation of standard Bayesian estimation methods for the estimation of the parameters and latent processes in the structural model. Our goal is to quantify the role of ambiguity shocks in the joint dynamics of output, unemployment, inflation and interest rates as well as the households' belief wedges associated with these variables. Compared to the analysis in Section 3, the impact of these shocks on the economy is restricted through the structure of the model, and we use survey data as an additional source of information to aid identification. 
The crucial force driving the role of ambiguity concerns in the model is the interaction of ambiguity shocks with other sources of exogenous variation under the households' worst-case model. Therefore, in order to make the estimation tractable and transparent, we calibrate a subset of parameters to the posterior estimates from Christiano et al. (2015) listed in the bottom part of Table 1, and focus our estimation on the parameter vector

$$
\left\{\rho_{R}, r_{\pi}, r_{y}, \sigma_{R}, \sigma_{A}, \rho_{\Psi}, \sigma_{\Psi}, \rho_{f}, \sigma_{f}, \sigma_{\Delta, \pi}, \sigma_{\Delta, u}, \sigma_{\Delta, d \mathcal{Y}}\right\}
$$

which consists of parameters associated with the monetary policy rule and the underlying shock processes. The last three parameters are the standard deviations on the measurement errors. Our priors for the monetary policy rule coefficients and stochastic processes for technology and monetary policy shocks in line with those in Christiano et al. (2015).

As in Section 3, we use data on GDP growth, the unemployment rate, Federal Funds rate, inflation rate, the GDP growth wedge, unemployment wedge and inflation wedge with iid measurement errors on the three wedges. The first part of Table 1 summarizes the results of our estimation. ${ }^{9}$

\section{Understanding the role of ambiguity shocks}

Table 2 provides the variance decomposition for key macroeconomic variables and the belief wedges. Despite the noise in the survey answers, the estimated model picks up a meaningful amount of common variation from the survey answers (up to 22\%), and has a substantial impact on key macroeconomic variables. In this section, we analyze in detail the mechanism through which ambiguity shocks propagate into the economy. In Appendix G we provide additional discussion and conduct a set of robustness checks with alternative belief wedge specifications that corroborate our quantitative results.

\subsection{Belief wedges and the worst-case model}

Figure 4 depicts the impulse responses for the ambiguity shock $w_{t}^{f}$. A one-standard deviation increase in ambiguity leads to a fall of about $2 \%$ in output growth on impact, and to almost a 1 percentage point increase in the unemployment rate that peaks after about four quarters. Inflation increases in the moment of the impact of the shock as well. These responses are larger than those estimated using the factor model from Section 3 and depicted in Figure 3 but they tell the same qualitative story - an increase in ambiguity is contractionary. The bottom row of Figure 4 shows that households become pessimistic about GDP growth, expecting even lower growth than the one predicted using the impulse response from the top left panel. Households also overpredict inflation and unemployment. All shifts in beliefs are again consistent with the factor model findings from Section 3.

\footnotetext{
${ }^{9}$ The details of the data construction are in Appendix C. We estimate the model using a Metropolis-Hastings algorithm. We take five chains with different initial draws and make 20,000 draws in each chain. The first 10,000 draws of each chain are dropped.
} 


\begin{tabular}{|c|c|c|c|}
\hline \multicolumn{2}{|c|}{ Parameter } & $\begin{array}{c}\text { Prior } \\
\text { D(Mean,Std }) \\
\end{array}$ & $\begin{array}{c}\text { Posterior } \\
\text { Mean(90\% HPD) }\end{array}$ \\
\hline \multicolumn{4}{|c|}{ Monetary policy rule } \\
\hline$\rho_{R}$ & Smoothing & $\mathcal{B}(0.7,0.1)$ & $0.79(0.77,0.81)$ \\
\hline$r_{\pi}$ & Inflation & $\mathcal{G}(1.5,0.1)$ & $1.67(1.59,1.77)$ \\
\hline$r_{y}$ & Output & $\mathcal{G}(0.06,0.01)$ & $0.03(0.02,0.03)$ \\
\hline \multicolumn{4}{|c|}{ Shock processes } \\
\hline$\rho_{f}$ & $\mathrm{AR}(1)$ ambiguity & $\mathcal{B}(0.5,0.1)$ & $0.87(0.85,0.89)$ \\
\hline$\sigma_{f}$ & Std. dev. ambiguity shock & $\mathcal{G}(0.1,0.1)$ & $0.02(0.01,0.02)$ \\
\hline $100 \sigma_{A}$ & Std. dev. neutral tech. shock & $\mathcal{G}(0.1,1.0)$ & $0.86(0.81,0.91)$ \\
\hline$\rho_{\Psi}$ & $\operatorname{AR}(1)$ invest. tech. & $\mathcal{B}(0.5,0.1)$ & $0.29(0.25,0.33)$ \\
\hline $100 \sigma_{\Psi}$ & Std. dev. invest. tech. shock & $\mathcal{G}(0.1,1.0)$ & $2.88(2.56,3.18)$ \\
\hline $100 \sigma_{R}$ & Std. dev. monetary policy shock & $\mathcal{G}(0.2,1.0)$ & $0.24(0.22,0.26)$ \\
\hline \multicolumn{4}{|c|}{ Measurement errors } \\
\hline $100 \sigma_{\Delta, d \mathcal{Y}}$ & GDP growth wedge & $\mathcal{I} \mathcal{G}(0.8,0.4)$ & $0.40(0.36,0.43)$ \\
\hline $100 \sigma_{\Delta, u}$ & Unemployment wedge & $\mathcal{I G}(0.4,0.2)$ & $0.17(0.15,0.19)$ \\
\hline $100 \sigma_{\Delta, \pi}$ & Inflation wedge & $\mathcal{I} \mathcal{G}(0.6,0.3)$ & $0.29(0.26,0.32)$ \\
\hline \multicolumn{4}{|c|}{ Calibrated parameters } \\
\hline$\beta$ & Discount factor & 0.9968 & - \\
\hline $100 \delta_{k}$ & Physical capital depreciation rate & 2.5 & - \\
\hline$\chi$ & Calvo price stickiness & 0.75 & - \\
\hline$\lambda$ & Price markup & 1.42 & - \\
\hline$\rho$ & Job survival probability & 0.9 & - \\
\hline$M$ & Max. bargaining rounds per quarter & 60 & - \\
\hline$\sigma$ & Matching function elasticity & 0.55 & - \\
\hline $100 \delta$ & Probability of bargaining breakup & 0.19 & - \\
\hline $400 \bar{\mu}$ & Output growth per capita & 1.7 & - \\
\hline$D$ & Replacement ratio & 0.37 & - \\
\hline $100 \eta_{h}$ & Hiring costs & 0.46 & - \\
\hline $100 \eta_{s}$ & Vacancy costs & 0.03 & - \\
\hline $400 \bar{\mu}_{\Psi}$ & Investment per capital growth rate & 1.2 & - \\
\hline $400 \bar{\pi}$ & Inflation rate & 2.5 & - \\
\hline$g$ & Government consumption to output & 0.2 & - \\
\hline$b$ & Consumption habit & 0.8 & - \\
\hline$\alpha$ & Capital share & 0.26 & - \\
\hline
\end{tabular}

Table 1: Structural model estimated and calibrated parameters. The priors $\mathcal{B}(\mu, \sigma), \mathcal{G}(\mu, \sigma)$, and $\mathcal{I} \mathcal{G}(\mu, \sigma)$ denote Beta, Gamma and Inverse-Gamma distributions with mean $\mu$ and standard deviation $\sigma$. 


\begin{tabular}{ll|rrrrr} 
Variable & & $w^{A}$ & $w^{\Psi}$ & $w^{R}$ & $w^{f}$ & $\begin{array}{r}\text { meas. } \\
\text { error }\end{array}$ \\
\hline $\log \left(\mathcal{Y}_{t} / \mathcal{Y}_{t-1}\right)$ & GDP growth & 38.4 & 36.1 & 0.8 & 24.8 & - \\
$u_{t}$ & Unemployment & 41.1 & 45.0 & 0.4 & 13.6 & - \\
$\pi_{t}$ & Inflation & 53.2 & 44.1 & 0.1 & 2.6 & - \\
$R_{t}$ & Nominal interest & 46.7 & 46.0 & 5.5 & 1.9 & - \\
$c_{t}-\log \Phi_{t}$ & Consumption & 58.0 & 6.0 & 0.3 & 35.7 & - \\
$i_{t}-\log \left(\Phi_{t} \Psi_{t}\right)$ & Investment & 70.2 & 21.7 & 0.3 & 7.8 & - \\
$\eta_{t}$ & Hiring rate & 38.2 & 38.9 & 1.1 & 21.8 & - \\
$V_{t}-\log \Phi_{t}$ & Continuation value & 67.3 & 6.9 & 0.2 & 25.6 & - \\
$\bar{\Delta}_{t}^{(4)}(d \mathcal{Y})$ & GDP growth wedge & - & - & - & 16.4 & 83.6 \\
$\bar{\Delta}_{t}^{(4)}(u)$ & Unemployment wedge & - & - & - & 6.3 & 93.7 \\
$\bar{\Delta}_{t}^{(4)}(\pi)$ & Inflation wedge & - & - & - & 22.0 & 78.0
\end{tabular}

Table 2: Structural model variance decomposition at the posterior modes. All values are in percent.

GDP growth

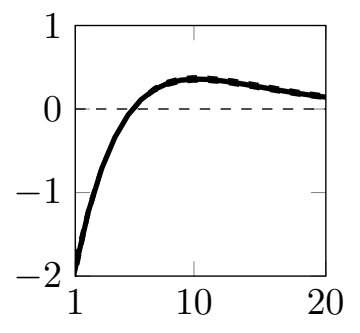

GDP growth wedge

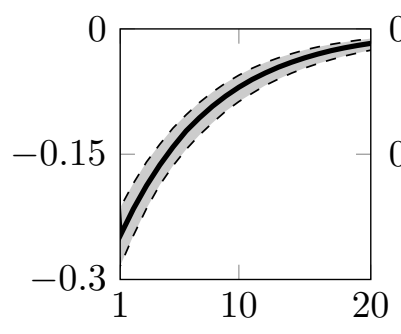

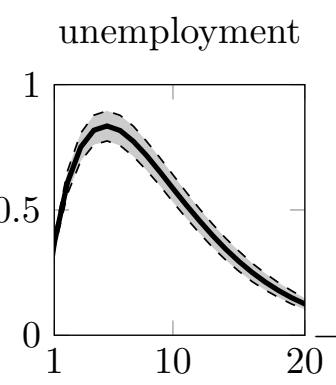

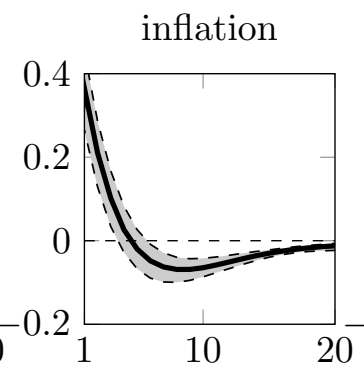

unemp. wedge

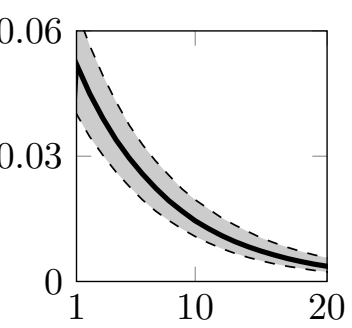

inflation wedge

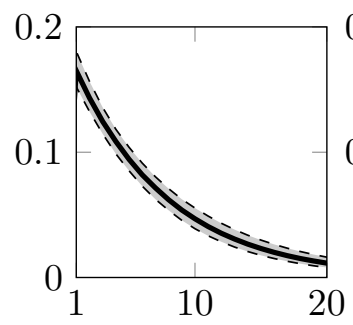

interest rate

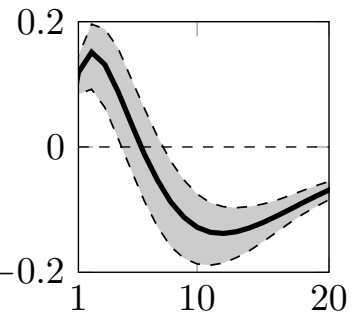

ambiguity

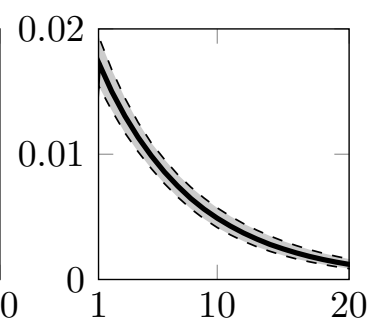

Figure 4: Bayesian impulse response functions to the ambiguity shock $w^{f}$ in the structural model. The responses of GDP growth, inflation rate and interest rate are reported in annualized percent, and unemployment rate is in percentage points. The solid lines indicate median estimates, while the dashed line correspond to the 10th and 90th percentile error bands. Horizontal axis in quarters.

The structural model allows us to explain the economic mechanism underlying the role of the ambiguity shock. This shock affects households' concerns about model misspecification and therefore alters their worst-case model $\widetilde{P}$. In order to understand the impact of the ambiguity shock, it is therefore useful to distinguish between the impulse responses under the data-generating process $P$ and under the worst-case model $\widetilde{P}$. The former impulse responses are those observed by the rational econometrician, while the latter are perceived by the household in the model.

Figure 5 compares both responses to the ambiguity shock $w_{t}^{f}$. After an increase in ambiguity, 
GDP growth

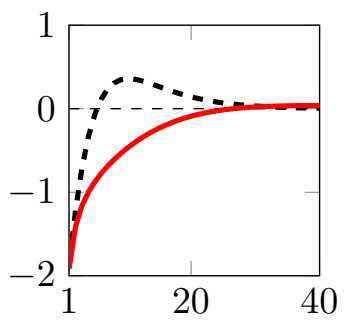

neutral tech.

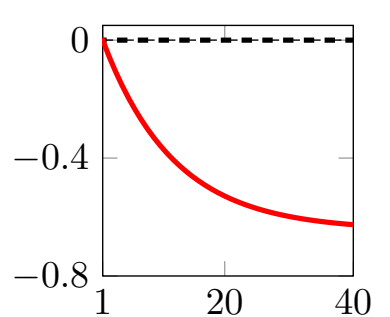

unemployment

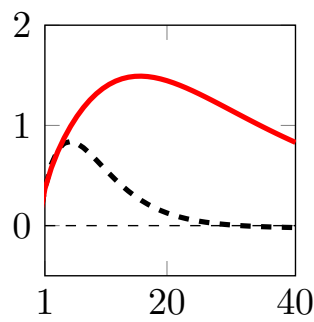

investment tech.

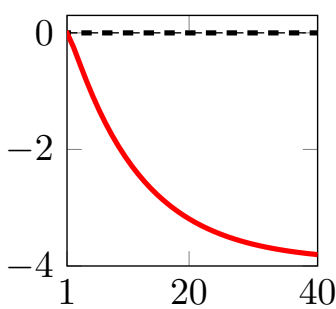

inflation

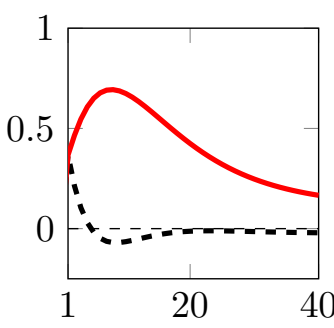

monetary policy

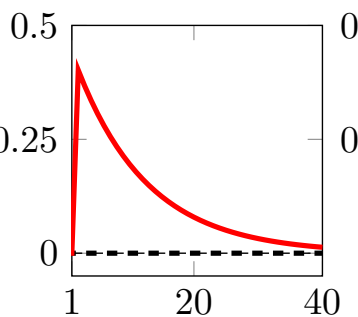

interest rate

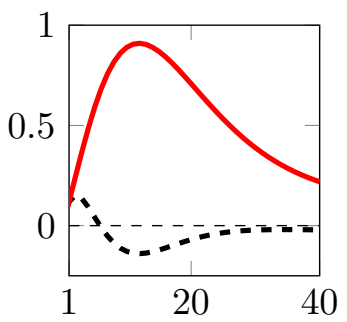

ambiguity

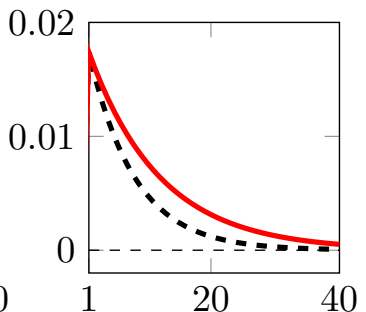

Figure 5: Impulse response functions to the ambiguity shock $w^{f}$ in the structural model under the data-generating measure $P$ (black dashed line) and the worst-case model $\widetilde{P}$ (red solid line). Impulse response functions evaluated at the mode of the posterior distribution. Horizontal axis in quarters.

the households' worst-case model becomes more pessimistically biased. In line with expression (12), the worst-case impulse responses are more persistent - households expect the adverse effects of an ambiguity increase on the economy to persist longer.

The bottom row of Figure 5 depicts the impulse responses for the individual exogenous shocks in the model. The dashed line in the bottom right panel depicts the response of the belief process $\theta_{t}=f_{t}$ to the innovation $w_{t}^{f}$ under the data-generating measure $P$. The household indeed expects that under the worst case model (red solid line) the ambiguity increase will be more persistent.

Since the individual exogenous shocks are uncorrelated, there is no response of the technology processes or the monetary policy shock to the innovation $w_{t}^{f}$ — the dashed lines in the corresponding panels are flat. The story under the worst-case model is very different and critical to the understanding of the endogenous response of the macroeconomy to the ambiguity shock. Under the worst-case model, the household believes that the shocks are correlated in an adverse way. An increase in ambiguity worsens households' expectations about the future path of the neutral and investment-specific technology, and leads households to expect a monetary tightening.

This particular correlation structure arises because these three innovations to the exogenous processes all affect the continuation value $V_{t}$. In times with low neutral and investment-specific productivity growth, and times in which the economy is hit by an exogenous monetary tightening through the shock $w_{t}^{R}$ are bad times, with a low continuation value $V_{t}$. Moreover, the continuation value recursion (17) indicates that these bad times must be generated by low levels of current and future consumption under the households' worst-case model. The first panel Figure 6 indeed 

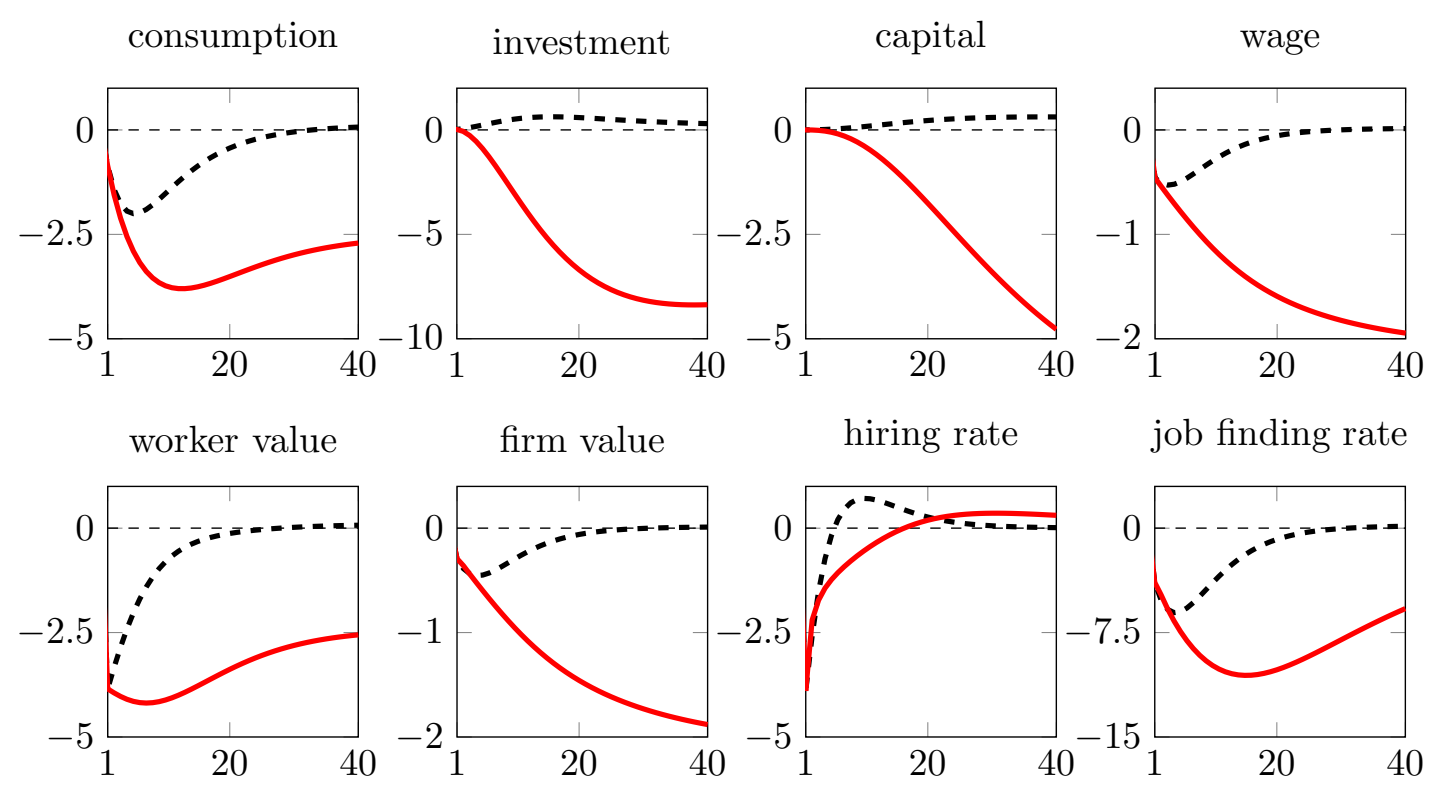

job finding rate

Figure 6: Impulse response functions to the ambiguity shock $w^{f}$ in the structural model under the data-generating measure $P$ (black dashed line) and the worst-case model $\widetilde{P}$ (red solid line). Impulse response functions evaluated at the mode of the posterior distribution. Horizontal axis in quarters.

confirms this intuition - the household that faces an increase in ambiguity forecasts a large and very persistent drop in consumption.

The second panel in Figure 6 shows that the increase in ambiguity is also accompanied by an increase in expected investment activity under the data-generating measure $P$, reflecting intertemporal consumption smoothing. However, the households' worst-case expectations are much more pessimistic, and this strong effect arises from the large distortion of the investment specific productivity shock. Under our estimated parameterization (see Table 1), the investment specific shock is volatile, which allows for large pessimistic distortions that are hard to distinguish statistically and therefore not penalized heavily by the entropy penalty in (17). At the same time, the shock has a large impact on the productivity of the investment technology and future capital accumulation, which in turn strongly affects future output and consumption paths. Combining these two effects leads to the strong adverse response of investment and capital under the worst-case model.

The effect on the inflation rate comes from a balance of two forces. Lower contemporaneous aggregate demand pushes the intermediate goods producers that change prices to set them to lower levels. At the same time, expectations of lower productivity imply higher marginal costs and this pushes current and future prices upwards. At our current estimates, the net effect of an increase in ambiguity is a higher equilibrium inflation rate in the year after the impact of the ambiguity shock. At the same time, the response of the inflation wedge is positive, implying that the worst-case model is biased toward an even higher inflation rate in the future. 


\subsection{Labor market dynamics}

These pessimistic expectations interact in crucial ways through the frictional labor market whose dynamics is captured by impulse responses in Figure 6. With search and matching rigidities, hiring and bargaining decisions are based on the value of the discounted future surplus generated by a match. Both firms and workers inherit the representative household's beliefs to make future forecasts when they compute their respective continuation values. Lower expected productivity and higher expected interest rates lower the value of the match from the perspective of the worst-case beliefs shared by the worker-firm pair. This lowers equilibrium hiring rates, and lower employment also implies lower output. Equilibrium wages also fall, reflecting the decline in the surplus that is particularly large and persistent under the worst-case model. All these effects are captured by the remaining responses in Figure 6.

This channel induced by fluctuations in household's ambiguity concerns is a potent source of fluctuations in the labor market. The variance decomposition in Table 2 reveals that ambiguity shocks drive a substantial portion of the overall variation in labor market variables, for instance more than $20 \%$ of the variation in the hiring rate.

Our results also provide a structural explanation for the discount rate shocks in Hall (2015) and their role in the labor market dynamics. When ambiguity concerns increase, agents evaluate more pessimistically those cash flows that positively correlate with households' continuation values. Since the match surplus is procyclical, it is effectively discounted at a higher rate. Contrary to Hall (2015), the discounting arises endogenously through the optimization problem of the robust household, and the time-variation is empirically linked to the observable survey data.

\subsection{The role of permanent shocks}

In the model description, we emphasized the unit root specification of the technology processes as a source of large and volatile belief wedges. An innovation to a persistent technology process affects productivity for a long period of time, and therefore leads to a large movement in today's continuation value $V_{t}$. As a consequence, the belief distortion $m_{t+1}$ in (19) is sufficiently volatile, generating a large wedge between the data-generating process and the worst-case belief whose magnitude is controlled by fluctuations in the belief shock $\theta_{t}$. This persistence effect is exacerbated when represented under the worst-case belief.

We also estimated a version of the model with stationary technology shocks. In that version, the magnitude of the belief distortions pushed the estimation of the dynamics of the belief shock into the non-stationary region under the worst-case model. Stationary fluctuations in the model were not sufficient to generate a sufficiently large distance between the data-generating and worst-case model.

The importance of persistent shocks is analogous to the role of 'long-run risk' shocks under recursive preferences of the Epstein and Zin (1989) type. Under these preferences, the stochastic discount factor is directly exposed to fluctuations in the continuation value $V_{t}$, generating aversion to persistence for parameterizations where risk aversion is larger than the inverse of the intertemporal 


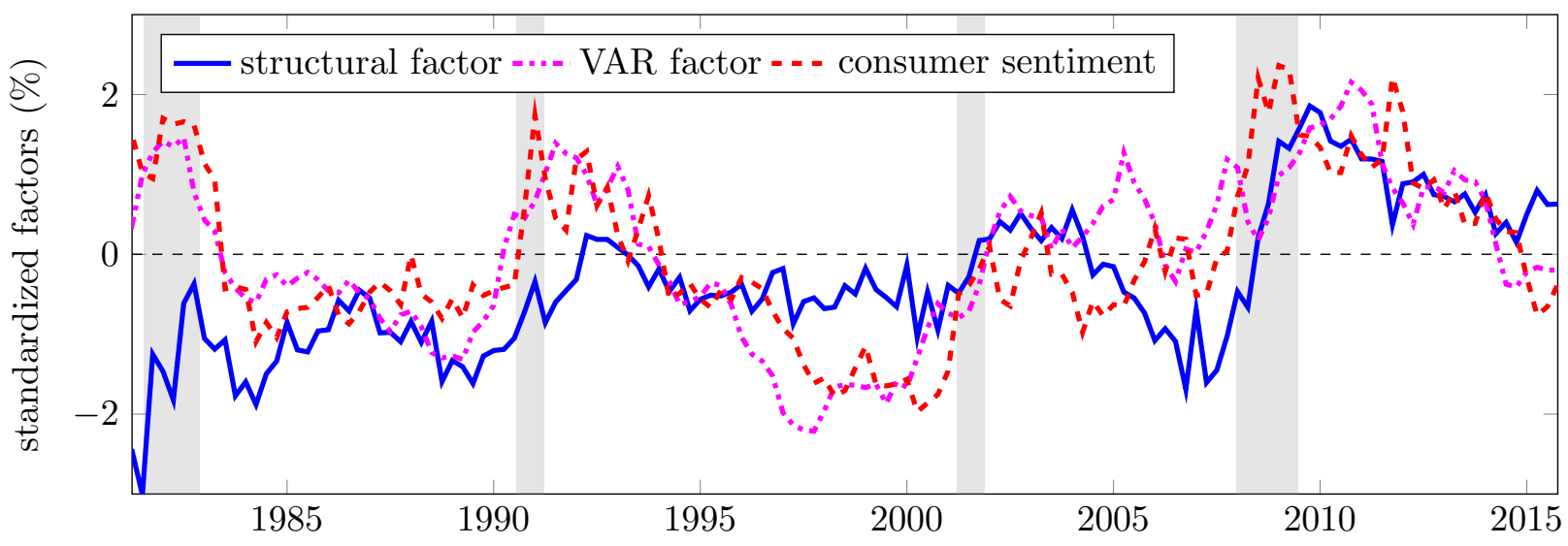

Figure 7: A comparison of the extracted ambiguity factor with the negative of the Michigan Survey measure of contemporaneous Index of Consumer Sentiment. The solid blue line is the smoothed factor from the structural model, the purple dash-dotted line the smoothed factor from the VAR specification. All data series are standardized.

elasticity of substitution. The crucial difference lies in the fact that an agent endowed with recursive preferences understands aversion to uncertainty as a risk adjustment, not a belief distortion that is reflected in the survey responses.

\subsection{Time-series variation}

While the ambiguity factor $f_{t}$ is treated as unobservable to the econometrician in both the factor and structural model, we can extract the filtered path for $f_{t}$ and compare it to observable statistics to see whether fluctuations in ambiguity concerns are reflected in other time series. In Figure 7 we plot the extracted series for the ambiguity factor obtained from the factor model and the structural model, along with the negative of the Index of Consumer Sentiment reported by the Michigan Survey. ${ }^{10}$

All three series are highly correlated and increase during recessions, attesting to a consistent narrative of how ambiguity fluctuations interact with business cycle dynamics. Particularly notable is the large increase in the extracted factor during the 2007-2009 recession and a subsequent slow decline. Through the lens of the model, pessimistic expectations connected to a prolonged period of increased ambiguity played a role in the slow recovery following the recession.

\subsection{Mean distortions and detection error probabilities}

So far we focused on the role of ambiguity shocks in business cycle fluctuations. The model also implies restrictions on the relative magnitude of the mean biases in the survey responses. We set the mean level of ambiguity concerns $E\left[\theta_{t}\right]=\bar{\theta} \cdot \bar{f}$ to minimize the distance between the mean

\footnotetext{
${ }^{10}$ In Figure 12 in Appendix G, we also plot the time paths for the ambiguity factor and implied belief wedges estimated using alternative belief wedge specifications that confirm the cyclical nature of the ambiguity factor and associated belief wedges, providing additional robustness checks to our quantitative results.
} 


\begin{tabular}{l|rr|r} 
& \multicolumn{2}{|c|}{ mean wedges } & forecast \\
& data & model & error std. \\
\hline GDP growth & -1.73 & -1.76 & 1.88 \\
Unemployment & 0.55 & 0.35 & 0.46 \\
Inflation & 1.01 & 1.19 & 1.24
\end{tabular}

Table 3: Mean belief wedges $\Delta_{t}^{(4)}$ in the data and implied by the structural model for the 4-quarter ahead forecasts of inflation, unemployment and GDP growth, compared to the standard deviation of the forecast errors in the factor model. The mean level of ambiguity concerns $E\left[\theta_{t}\right]=\bar{\theta} \cdot \bar{f}$ is found by minimizing the sum of squared percentage deviations in the mean belief wedges in the model and in the data. Normalizing $\bar{\theta}=1$, we find $\bar{f}=0.1076$.

belief wedges implied by the model and measured in household survey responses. The results are summarized in Table 3. The relative magnitude of the model-implied mean belief wedges concerning inflation, unemployment and GDP growth forecasts $(1.19 \%, 0.55 \%$ and $-1.76 \%$, respectively) is quite close to that in the data $(1.01 \%, 0.55 \%$ and $-1.73 \%$, respectively). This is remarkable because we estimated the model using fluctuations in the deviation of the belief wedges from their long-run averages, without utilizing information on the mean wedges in any way.

Anderson et al. (2003) and Hansen and Sargent (2011) advocate a different way of calibrating the level of ambiguity concerns that is based on the statistical distinguishability of the benchmark and worst-case model. They consider the likelihood ratio

$$
l^{T}=\log L_{W}^{T}-\log L_{B}^{T}
$$

where $L_{W}^{T}$ and $L_{B}^{T}$ are the likelihoods of a data sample of length $T$ under the worst-case (W) and benchmark (B) models, respectively. The probability of a classification error committed using the likelihood ratio statistic for a data sample drawn from the benchmark model is

$$
p_{B}^{T}=P\left(l^{T}>0 \mid B\right),
$$

and, vice versa,

$$
p_{W}^{T}=P\left(l^{T}<0 \mid W\right) .
$$

The detection error probability is then defined as

$$
p^{T}=\frac{1}{2}\left(p_{B}^{T}+p_{W}^{T}\right)
$$

The (constant) penalty parameter $\theta$ from equation (4) that captures the level of ambiguity concerns is then fine-tuned to achieve a desired level of detection error for a data sample of a given length.

We pinned down the level of ambiguity concerns $E\left[\theta_{t}\right]$ using the mean biases in household survey forecasts. In Figure 8, we plot the implied detection error probabilities as a function of the length of the data sample. Details of the calculations are provided in Appendix F. 

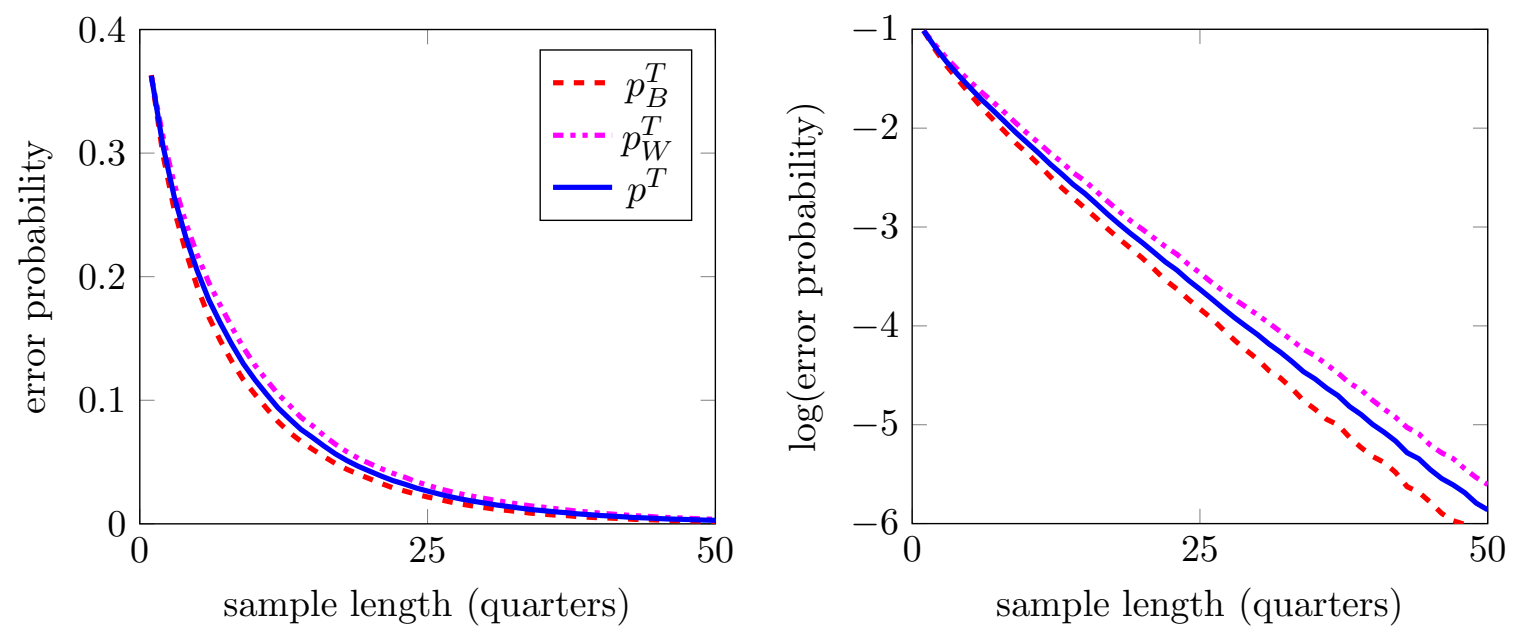

Figure 8: Detection error probabilities in the structural model as a function of data sample length. Probabilities constructed by simulating 100,000 samples from the benchmark and worst-case model, respectively, and constructing sample versions of classification errors $p_{B}^{T}$ and $p_{W}^{T}$, and the detection error probability $p^{T}$.

The calculation reveals that the implied detection error probabilities are relatively low - it takes about 5 years of data to tell the benchmark and worst-case model apart at a $5 \%$ error probability, and about 9 years to achieve a 1\% detection error. Viewed through the lens of the structural model, the pessimistic biases embedded in the household survey answers are quite strong, implying that households require a very high level of confidence to discard a model as implausible. Table 3 shows that the mean belief wedges for the 4-quarter ahead forecasts are comparable to one standard deviation of the forecast error computed using the factor model.

Detection error calibrations in the existing literature are typically substantially more conservative than our findings. For instance, Barillas et al. (2009) or Bidder and Smith (2012) choose detection error probabilities in the range of $1 \%-5 \%$ for data samples spanning about 50 years to fit the magnitude of the equity premium. Although these detection error probabilities seem to be low, our direct evidence from household surveys suggests that these calibrations may still be too conservative when dealing with macroeconomic applications and households from typical populations.

\section{Conclusion}

We develop a framework in which time-variation in ambiguity perceived by households generates fluctuations in aggregate dynamics of the macroeconomy. The framework is based on an extension of the robust preference model that introduces shocks to agents' concerns about model misspecification. We identify these ambiguity shocks using survey data from the University of Michigan Surveys of Consumers and the Survey of Professional Forecasters. We show that in the data and in an estimated business cycle model, the ambiguity shocks are a potent source of variation in labor 
market variables.

The developed solution techniques are applicable to an extensive class of models that feature agents with robust preferences. The linear approximation that captures time-variation in ambiguity concerns is easy to implement and estimate, which immensely facilitates quantitative work in dynamic equilibrium models with robust agents.

In the paper, we consider ambiguity shocks generated by an exogenous source and identified through fluctuations in biases of households' survey responses. However, our framework also allows for fluctuations in ambiguity concerns caused by movements in endogenous variables in the model - for instance, ambiguity could increase in response to an increase in aggregate unemployment. We leave the structural modeling and empirical identification of such mechanisms for future research.

The structural interpretation of ambiguity shocks identified in our framework opens new directions for policy analysis under ambiguity, extending the work of Hansen and Sargent (2012), Adam and Woodford (2012), Karantounias (2013), Orlik and Presno (2013), or Kwon and Miao (2013). In parallel work, we study the implications of this framework for optimal monetary policy. A monetary authority facing households endowed with robust preferences infers that policy changes lead to endogenous changes in the worst-case model. The choice of optimal policy therefore involves explicit management of households' expectations by the monetary authority. Reciprocally, belief wedges in survey responses are structurally tied to the dynamics of equilibrium allocations in the model, and serve the useful purpose of providing the monetary authority with information about the decision-making process of private agents. 


\section{Appendix}

\section{A Distorted beliefs in the one-factor model}

Let $\left(\Omega,\left\{\mathcal{F}_{t}\right\}_{t=0}^{\infty}, P\right)$ be the probability space generated by the innovations of model (1). The subjective probability measure $\widetilde{P}$ is formally defined by specifying a strictly positive martingale $M$ with one-period increment

$$
m_{t+1}=\frac{M_{t+1}}{M_{t}}=\exp \left(-\frac{1}{2}\left|\nu_{t}\right|^{2}+\nu_{t}^{\prime} w_{t+1}\right) .
$$

We then have $\widetilde{E}_{t}\left[w_{t+1}\right]=\nu_{t}$. Using the notation $x_{t}=\left(y_{t}^{\prime}, f_{t}\right)^{\prime}$, the factor structure (2)-(3) of households' expectations is obtained by imposing the restriction

$$
\nu_{t}=\bar{H}+H F x_{t}
$$

where $F=\left(F_{y}, F_{f}\right)$ in an $1 \times n$ vector and $H, \bar{H}$ are $k \times 1$ vectors.

Let $\zeta_{t}=Z x_{t}$ be the vector of variables for which we have observable data on households' expectations where $Z$ is a selection matrix. Here, we derive results for more general dynamics that is in line with the non-stationary model from Section 4 . Specifically, we assume that

$$
\begin{aligned}
\zeta_{t} & =Z x_{t}=Z \widehat{x}_{t}+z_{t} \\
\widehat{x}_{t+1} & =\psi_{q}+\psi_{x} \widehat{x}_{t}+\psi_{w} w_{t+1} \\
z_{t+1}-z_{t} & =\phi_{q}+\phi_{x} \widehat{x}_{t}+\phi_{q} w_{t+1} .
\end{aligned}
$$

The process $z_{t}$ introduces an additional component of the dynamics that has stationary growth rates. The factor model framework from Section 3 is obtained by setting the matrices $\bar{H}, \psi_{q}, \psi_{x}, \psi_{w}$ and $\phi_{q}$ to zero, in which case

$$
x_{t+1}=\psi_{x} x_{t}+\psi_{w} w_{t+1}
$$

is a concise form for (1).

We are interested in $\tau$-period ahead belief wedges

$$
\Delta_{t}^{(\tau)}=\widetilde{E}_{t}\left[\zeta_{t+\tau}\right]-E_{t}\left[\zeta_{t+\tau}\right]
$$

Guess that

$$
\begin{aligned}
E_{t}\left[\zeta_{t+\tau}-\zeta_{t}\right] & =G_{x}^{(\tau)} \widehat{x}_{t}+G_{0}^{(\tau)} \\
\widetilde{E}_{t}\left[\zeta_{t+\tau}-\zeta_{t}\right] & =\widetilde{G}_{x}^{(\tau)} \widehat{x}_{t}+\widetilde{G}_{0}^{(\tau)}
\end{aligned}
$$

where $G_{x}^{(\tau)}, G_{0}^{(\tau)}, \widetilde{G}_{x}^{(\tau)}$ and $\widetilde{G}_{0}^{(\tau)}$ are conformable matrix coefficients with initial conditions

$$
G_{0}^{(\tau)}=\widetilde{G}_{0}^{(\tau)}=0_{n \times 1} \quad G_{x}^{(\tau)}=\widetilde{G}_{0}^{(\tau)}=0_{n \times n} .
$$


We can then establish a recursive formula for the expectations under the data-generating measure

$$
\begin{aligned}
G_{x}^{(\tau)} \widehat{x}_{t}+G_{0}^{(\tau)}= & E_{t}\left[\zeta_{t+\tau}-\zeta_{t}\right]= \\
= & E_{t}\left[Z\left(x_{t+1}-x_{t}\right)+G_{x}^{(\tau-1)} \widehat{x}_{t+1}+G_{0}^{(\tau-1)}\right] \\
= & G_{0}^{(\tau-1)}+\phi_{q}+\left(Z+G_{x}^{(\tau-1)}\right) \psi_{q}+\left[\left(Z+G_{x}^{(\tau-1)}\right) \psi_{x}+\left(\phi_{x}-Z\right)\right] \widehat{x}_{t} \\
& +\left(\left(Z+G_{x}^{(\tau-1)}\right) \psi_{w}+\phi_{w}\right) E_{t}\left[w_{t+1}\right]
\end{aligned}
$$

Since $E_{t}\left[w_{t+1}\right]=0$, we obtain

$$
\begin{aligned}
G_{x}^{(\tau)} & =\left(Z+G_{x}^{(\tau-1)}\right) \psi_{x}+\left(\phi_{x}-Z\right) \\
G_{0}^{(\tau)} & =G_{0}^{(\tau-1)}+\phi_{q}+\left(Z+G_{x}^{(\tau-1)}\right) \psi_{q} .
\end{aligned}
$$

Under the subjective measure, the derivation is unchanged, except the last line in (22) that now involves the subjective expectation $\widetilde{E}_{t}\left[w_{t+1}\right]=\bar{H}+H F \widehat{x}_{t}$. Then

$$
\begin{aligned}
& \widetilde{G}_{x}^{(\tau)}=\left(Z+\widetilde{G}_{x}^{(\tau-1)}\right) \psi_{x}+\left(\phi_{x}-Z\right)+\left(\left(Z+\widetilde{G}_{x}^{(\tau-1)}\right) \psi_{w}+\phi_{w}\right) H F \\
& \widetilde{G}_{0}^{(\tau)}=\widetilde{G}_{0}^{(\tau-1)}+\phi_{q}+\left(Z+\widetilde{G}_{x}^{(\tau-1)}\right) \psi_{q}+\left(\left(Z+\widetilde{G}_{x}^{(\tau-1)}\right) \psi_{w}+\phi_{w}\right) \bar{H}
\end{aligned}
$$

Consequently

$$
\Delta_{t}^{(\tau)}=\left(\widetilde{G}_{x}^{(\tau)}-G_{x}^{(\tau)}\right) \widehat{x}_{t}+\widetilde{G}_{0}^{(\tau)}-G_{0}^{(\tau)} .
$$

In the case considered in Section 3 when $\bar{H}, \phi_{q}, \phi_{x}, \phi_{w}$ and $\phi_{q}$ are all zero, we get explicit expressions

$$
\begin{aligned}
& G_{x}^{(\tau)}=Z\left(\psi_{x}\right)^{\tau} \\
& G_{0}^{(\tau)}=Z \sum_{i=0}^{\tau-1}\left(\psi_{x}\right)^{i} \psi_{q}=Z\left(I-\psi_{x}\right)^{-1}\left(I-\left(\psi_{x}\right)^{\tau}\right) \psi_{q} \\
& \widetilde{G}_{x}^{(\tau)}=Z\left(\psi_{x}+\psi_{w} H F\right)^{\tau} \\
& \widetilde{G}_{0}^{(\tau)}=Z \sum_{i=0}^{\tau-1}\left(\psi_{x}+\psi_{w} H F\right)^{i} \psi_{q}=Z\left(I-\left(\psi_{x}+\psi_{w} H F\right)\right)^{-1}\left(I-\left(\psi_{x}+\psi_{w} H F\right)^{\tau}\right) \psi_{q} .
\end{aligned}
$$

\section{B Series expansion of the worst-case model}

The linear approximation in this paper is an extension of the series expansion method used in Holmes (1995) or Lombardo (2010). Borovička and Hansen (2013, 2014) adapt the series expansion method to an approximation of models with robust preferences that feature a constant penalty. Here, we further extend this methodology to derive a linear approximation that allows for endogenously determined time-varying belief distortions. The critical step in the expansion lies in the joint perturbation of the shock vector $w_{t}$ and the penalty process $\theta_{t}$.

\section{B.1 Law of motion}

We start with the approximation of the model for the law of motion (5) with a sufficiently smooth policy rule $\psi$. We consider a class of models indexed by the scalar perturbation parameter $\mathrm{q}$ that scales the volatility 
of the shock vector $w_{t}$

$$
x_{t}(\mathbf{q})=\psi\left(x_{t-1}(\mathbf{q}), \mathbf{q} w_{t}, \mathbf{q}\right)
$$

and assume that there exists a series expansion of $x_{t}$ around $\mathbf{q}=0$ :

$$
x_{t}(\mathrm{q}) \approx \bar{x}+\mathrm{q} x_{1 t}+\frac{\mathrm{q}^{2}}{2} x_{2 t}+\ldots
$$

The processes $x_{j t}, j=0,1, \ldots$ can be viewed as derivatives of $x_{t}$ with respect to the perturbation parameter, and their laws of motion can be inferred by differentiating (23) $j$ times and evaluating the derivatives at $q=0$, assuming that $\psi$ is sufficiently smooth. Here, we focus only on the approximation up to the first order:

$$
\begin{aligned}
\bar{x} & =\psi(\bar{x}, 0,0) \\
x_{1 t} & =\psi_{x} x_{1 t-1}+\psi_{w} w_{t}+\psi_{q} .
\end{aligned}
$$

We begin with a case where the equilibrium dynamics of $x_{t}$ is stationary. Extensions to non-stationary environments are considered in Appendix B.6.

\section{B.2 Continuation values}

We now focus on the expansion of the continuation value recursion. Substituting the worst-case belief distortion (7) into the recursion (4) yields

$$
V_{t}=u\left(x_{t}\right)-\frac{\beta}{\theta_{t}} \log E_{t}\left[\exp \left(-\theta_{t} V_{t+1}\right)\right]
$$

We are looking for an expansion of the continuation value

$$
V_{t}(\mathrm{q}) \approx \bar{V}+\mathrm{q} V_{1 t}
$$

In order to derive the solution of the continuation value, we are interested in expanding the following perturbation of the recursion:

$$
V_{t}(\mathbf{q})=u\left(x_{t}(\mathbf{q}), \mathbf{q}\right)-\beta \frac{\mathbf{q}}{\bar{\theta}\left(\bar{x}+x_{1 t}\right)} \log E_{t}\left[\exp \left(-\frac{\bar{\theta}\left(\bar{x}+x_{1 t}\right)}{\mathbf{q}} V_{t+1}(\mathbf{q})\right)\right]
$$

Here, we utilized the fact that $\theta_{t}=\bar{\theta} x_{t} \approx \bar{\theta}\left(\bar{x}+x_{1 t}\right)$. More importantly, the perturbation scales jointly the volatility of the stochastic processes for $V_{t}$ and $u\left(x_{t}\right)$ with the magnitude of the penalty parameter $\theta_{t}$. In particular, the penalty parameter in the perturbation of equation (4) becomes q/ $\theta_{t}$ and decreases jointly with the volatility of the shock process. This assumption will imply that the benchmark and worst-case models do not converge as $\mathrm{q} \rightarrow 0$, and the linear approximation around a deterministic steady state yields a nontrivial contribution of the worst-case dynamics.

Using the expansion of the period utility function

$$
u\left(x_{t}(\mathbf{q}), \mathbf{q}\right) \approx \bar{u}+\mathbf{q} u_{1 t}=\bar{u}+\mathbf{q}\left(u_{x} x_{1 t}+u_{q}\right)
$$


we get the deterministic steady state (zero-th order) term by setting $\mathbf{q}=0$ :

$$
\bar{V}=(1-\beta)^{-1} \bar{u} .
$$

The first-order term in the expansion is derived by differentiating (27) with respect to q and is given by the recursion

$$
V_{1 t}=u_{1 t}-\beta \frac{1}{\bar{\theta}\left(\bar{x}+x_{1 t}\right)} \log E_{t}\left[\exp \left(-\bar{\theta}\left(\bar{x}+x_{1 t}\right) V_{1 t+1}\right)\right]
$$

Since $\bar{x}$ is constant and $x_{1 t}$ has linear dynamics (24), we hope to find linear dynamics for $V_{1 t}$ as well. Since $u_{t}=u\left(x_{t}\right)$, we can make the guess that $V_{t}^{i}(\mathbf{q})=V^{i}\left(x_{t}(\mathbf{q}), \mathbf{q}\right)$ which leads to the following expressions for the derivative of $V_{t}$ :

$$
V_{1 t}=V_{x} x_{1 t}+V_{q}
$$

Using this guess and comparing coefficients, equation (28) leads to a pair of algebraic equations for the unknown coefficients $V_{x}$ and $V_{q}$ :

$$
\begin{aligned}
V_{x} & =u_{x}-\frac{\beta}{2} V_{x} \psi_{w} \psi_{w}^{\prime} V_{x}^{\prime} \bar{\theta}+\beta V_{x} \psi_{x} \\
V_{q} & =u_{q}-\frac{\beta}{2} \bar{\theta} \bar{x} V_{x} \psi_{w} \psi_{w}^{\prime} V_{x}^{\prime}+\beta V_{x} \psi_{q}+\beta V_{q}
\end{aligned}
$$

The first from this pair of equations is a Riccati equation for $V_{x}$, which can be solved for given coefficients $\psi_{x}$ and $\psi_{w}$.

\section{B.3 Distortions}

With the approximation of the continuation value at hand, we can derive the expansion of the one-period belief distortion $m_{t+1}$ that defines the worst-case model relative to the benchmark model. As in (27), we scale the penalty parameter $\theta_{t}$ jointly with the volatility of the underlying shocks:

$$
m_{t+1}(\mathbf{q})=\frac{\exp \left(-\frac{1}{\mathrm{q}} \theta_{t} V_{t+1}(\mathbf{q})\right)}{E_{t}\left[\exp \left(-\frac{1}{\mathrm{q}} \theta_{t} V_{t+1}(\mathbf{q})\right)\right]} \approx m_{0, t+1}+\mathbf{q} m_{1, t+1}
$$

It turns out that in order to derive the correct first-order expansion, we are required to consider a second-order expansion of the continuation value

$$
V_{t}(\mathrm{q}) \approx \bar{V}+\mathrm{q} V_{1 t}+\frac{\mathrm{q}}{2} V_{2 t},
$$

although the term $V_{2 t}$ will be inconsequential for subsequent analysis. Substituting in expression (26) and noting that $\bar{V}$ is a deterministic term, we can approximate $m_{t+1}$ with

$$
m_{t+1}(\mathbf{q}) \approx \frac{\exp \left(-\bar{\theta}\left(\bar{x}+x_{1 t}\right)\left(V_{1 t+1}+\frac{\mathrm{q}}{2} V_{2 t+1}\right)\right)}{E_{t}\left[\exp \left(-\bar{\theta}\left(\bar{x}+x_{1 t}\right)\left(V_{1 t+1}+\frac{\mathrm{q}}{2} V_{2 t+1}\right)\right)\right]}
$$

Differentiating with respect to $q$ and evaluating at $q=0$, we obtain the expansion 


$$
\begin{aligned}
m_{0 t+1} & =\frac{\exp \left(-\bar{\theta}\left(\bar{x}+x_{1 t}\right) V_{1 t+1}\right)}{E_{t}\left[\exp \left(-\bar{\theta}\left(\bar{x}+x_{1 t}\right) V_{1 t+1}\right)\right]} \\
m_{1 t+1} & =-\frac{1}{2 \bar{\theta}\left(\bar{x}+x_{1 t}\right)} M_{0 t+1}\left[V_{2 t+1}-E_{t}\left[M_{0 t+1} V_{2 t+1}\right]\right]
\end{aligned}
$$

This expansion is distinctly different from the standard polynomial expansion familiar from the perturbation literature. First, observe that $m_{0 t+1}$ is not constant, as one would expect for a zeroth-order term, but nonlinear in $V_{1 t+1}$. However, since $E_{t}\left[m_{0 t+1}\right]=1$ we can thus treat $M_{0 t+1}$ as a change of measure that will adjust the distribution of shocks that are correlated with $m_{0 t+1}$. We will show that with Gaussian shocks, we can still preserve tractability. Further notice that $E_{t}\left[m_{1 t+1}\right]=0$.

The linear structure of $V_{1 t}$ also has an important implication for the worst-case distortion constructed from $m_{0 t+1}$. Substituting into (29) yields

$$
m_{0 t+1}=\frac{\exp \left(-\bar{\theta}\left(\bar{x}+x_{1 t}\right) V_{x} \psi_{w} w_{t+1}\right)}{E_{t}\left[\exp \left(-\bar{\theta}\left(\bar{x}+x_{1 t}\right) V_{x} \psi_{w} w_{t+1}\right)\right]} .
$$

This implies that for a function $f\left(w_{t+1}\right)$ with a shock vector $w_{t+1} \sim N(0, I)$,

$$
\widetilde{E}_{t}\left[f\left(w_{t+1}\right)\right]=E_{t}\left[m_{t+1} f\left(w_{t+1}\right)\right] \approx E_{t}\left[m_{0 t+1} f\left(w_{t+1}\right)\right]
$$

The distortion generating the $\widetilde{P}$ (worst-case) measure is therefore approximated by the 'zero-th' order term $m_{0 t+1}$, and the vector $w_{t+1}$ has the following distribution:

$$
w_{t+1} \sim N\left(-\bar{\theta}\left(\bar{x}+x_{1 t}\right)\left(V_{x} \psi_{w}\right)^{\prime}, I_{k}\right) .
$$

The mean of the shock is therefore time-varying and depends on the linear process $x_{1 t}$.

\section{B.4 Equilibrium conditions}

We assume that equilibrium conditions in our framework can be written as

$$
0=E_{t}\left[\widetilde{g}\left(x_{t+1}, x_{t}, x_{t-1}, w_{t+1}, w_{t}\right)\right]
$$

where $\widetilde{g}$ is an $n \times 1$ vector function and the dynamics for $x_{t}$ is implied by (5). This vector of equations includes expectational equations like Euler equations of the robust household, which can be represented using worst-case belief distortions $m_{t+1}$. We therefore assume that we can write the $j$-th component of $\tilde{g}$ as

$$
\widetilde{g}^{j}\left(x_{t+1}, x_{t}, x_{t-1}, w_{t+1}, w_{t}\right)=m_{t+1}^{\sigma_{j}} g^{j}\left(x_{t+1}, x_{t}, x_{t-1}, w_{t+1}, w_{t}\right) .
$$

where $\sigma_{j} \in\{0,1\}$ captures whether the expectation in the $j$-th equation is under the household's worst-case model. ${ }^{11}$ In particular, all nonexpectational equations and all equations not involving agents' preferences have $\sigma_{j}=0$. System (31) can then be written as

$$
0=E_{t}\left[\mathbb{M}_{t+1} g\left(x_{t+1}, x_{t}, x_{t-1}, w_{t+1}, w_{t}\right)\right]
$$

\footnotetext{
${ }^{11}$ The generalization to multiple agents with potentially heterogeneous concerns for robustness is straightforward, see the construction in Borovička and Hansen (2013).
} 
where $\mathbb{M}_{t+1}=\operatorname{diag}\left\{m_{t+1}^{\sigma_{1}}, \ldots, m_{t+1}^{\sigma_{n}}\right\}$ is a diagonal matrix of the belief distortions, and $g$ is independent of the robustness parameter $\theta_{t}$. As in Borovička and Hansen (2013), the zero-th and first-order expansions are

$$
\begin{aligned}
& 0=E_{t}\left[\mathbb{M}_{0 t+1} g_{0 t+1}\right]=g_{0 t+1} \\
& 0=E_{t}\left[\mathbb{M}_{0 t+1} g_{1 t+1}\right]+E_{t}\left[\mathbb{M}_{1 t+1} g_{0 t+1}\right]=E_{t}\left[\mathbb{M}_{0 t+1} g_{1 t+1}\right]
\end{aligned}
$$

where the last equality follows from $E_{t}\left[m_{1 t+1}\right]=0$.

For the first-order derivative of the equilibrium conditions, we have

$$
0=E_{t}\left[\mathbb{M}_{0 t+1} g_{1 t+1}\right]
$$

The first-order term in the expansion of $g_{t+1}$ is given by

$$
\begin{aligned}
g_{1 t+1}= & g_{x+} x_{1 t+1}+g_{x} x_{1 t}+g_{x-} x_{1 t-1}+g_{w+} w_{t+1}+g_{w} w_{t}+g_{q}= \\
= & {\left[\left(g_{x+} \psi_{x}+g_{x}\right) \psi_{x}+g_{x-}\right] x_{1 t-1}+\left[\left(g_{x+} \psi_{x}+g_{x}\right) \psi_{w}+g_{w}\right] w_{t}+} \\
& +\left(g_{x+} \psi_{x}+g_{x+}+g_{x}\right) \psi_{q}+g_{q}+\left(g_{x+} \psi_{w}+g_{w+}\right) w_{t+1}
\end{aligned}
$$

where symbols $x_{+}, x, x_{-}, w_{+}, w, q$ represent partial derivatives with respect to $x_{t+1}, x_{t}, x_{t-1}, w_{t+1}, w_{t}$ and $q$, respectively. Given the worst-case distribution of the shock vector (30), we can write

$$
\widetilde{E}_{t}\left[w_{t+1}\right]=-\left(V_{x} \psi_{w}\right)^{\prime} \bar{\theta}\left[\left(\bar{x}+\psi_{q}\right)+\psi_{x} x_{1 t-1}+\psi_{w} w_{t}\right]
$$

Let $[A]^{i}$ denote the $i$-th row of matrix $A$. Notice that

$$
\left[g_{x+} \psi_{w}+g_{w+}\right]^{i}\left(V_{x} \psi_{w}\right)^{\prime} \bar{\theta}
$$

is a $1 \times n$ vector. Construct the $n \times n$ matrix $\mathbb{E}$ by stacking these row vectors for all equations $i=1, \ldots, n$ :

$$
\mathbb{E}=\operatorname{stack}\left\{\sigma_{i}\left[g_{x+} \psi_{w}+g_{w+}\right]^{i}\left(V_{x} \psi_{w}\right)^{\prime} \bar{\theta}\right\}
$$

which contains non-zero rows for expectational equations under the worst-case model. Using matrix $\mathbb{E}$, we construct the conditional expectation of the last term in $g_{1 t+1}$ in (33). In particular

$$
\begin{aligned}
0= & E_{t}\left[\mathbb{M}_{0 t+1} g_{1 t+1}\right]= \\
= & {\left[\left(g_{x+} \psi_{x}+g_{x}\right) \psi_{x}+g_{x-}\right] x_{1 t-1}+\left[\left(g_{x+} \psi_{x}+g_{x}\right) \psi_{w}+g_{w}\right] w_{t}+} \\
& +\left(g_{x+} \psi_{x}+g_{x+}+g_{x}\right) \psi_{q}+g_{q}-\mathbb{E}\left[\left(\bar{x}+\psi_{q}\right)+\psi_{x} x_{1 t-1}+\psi_{w} w_{t}\right]
\end{aligned}
$$

Equation (32) is thus a system of linear second-order stochastic difference equations. There are wellknown results that discuss the conditions under which there exists a unique stable equilibrium path to this system (Blanchard and Kahn (1980), Sims (2002)). We assume that such conditions are satisfied. Comparing coefficients on $x_{1 t-1}, w_{t}$ and the constant term implies that

$$
\begin{aligned}
0 & =\left(g_{x+} \psi_{x}+g_{x}-\mathbb{E}\right) \psi_{x}+g_{x-} \\
0 & =\left(g_{x+} \psi_{x}+g_{x}-\mathbb{E}\right) \psi_{w}+g_{w} \\
0 & =\left(g_{x+} \psi_{x}+g_{x+}+g_{x}\right) \psi_{q}+g_{q}-\mathbb{E}\left(\bar{x}+\psi_{q}\right)
\end{aligned}
$$


These equations need to be solved for $\psi_{x}, \psi_{w}, \psi_{q}$ and $V_{x}$ where

$$
V_{x}=u_{x}-\frac{\beta}{2} V_{x} \psi_{w} \psi_{w}^{\prime} V_{x}^{\prime} \bar{\theta}+\beta V_{x} \psi_{x}
$$

and

$$
\mathbb{E}=\operatorname{stack}\left\{\sigma_{i}\left[g_{x+} \psi_{w}+g_{w+}\right]^{i}\left(V_{x} \psi_{w}\right)^{\prime} \bar{\theta}\right\}
$$

\section{B.5 Special case: $\theta_{t}$ is an exogenous $\operatorname{AR}(1)$ process}

In the application, we consider a special case that restricts $\theta_{t}$ to be an exogenous $\operatorname{AR}(1)$ process. With a slight abuse in notation, this restriction can be implemented by replacing the vector of variables $x_{t}$ with $\left(x_{t}^{\prime}, f_{t}\right)^{\prime}$ where $f_{t}$ is a scalar $\mathrm{AR}(1)$ process representing the time-variation in the concerns for robustness as an exogenously specified 'belief' shock:

$$
f_{t+1}=\left(1-\rho_{f}\right) \bar{f}+\rho_{f} f_{t}+\sigma_{f} w_{t+1}^{f} .
$$

The dynamics of the model then satisfies

$$
x_{t}=\psi\left(x_{t-1}, w_{t}, f_{t}\right)
$$

with steady state $\left(\bar{x}^{\prime}, \bar{f}\right)^{\prime}$. The vector $\bar{\theta}$ in (6) is then partitioned as $\bar{\theta}^{\prime}=\left(\bar{\theta}_{x}^{\prime}, \bar{\theta}_{f}\right)=\left(0_{1 \times n-1}, 1\right)$ and thus $\theta_{t}=f_{t}$. Constructing the first-order series expansion of (39), we obtain

$$
\left(\begin{array}{c}
x_{1 t+1} \\
f_{1 t+1}
\end{array}\right)=\left(\begin{array}{c}
\psi_{q} \\
0
\end{array}\right)+\left(\begin{array}{cc}
\psi_{x} & \rho_{f} \psi_{x f} \\
0 & \rho_{f}
\end{array}\right)\left(\begin{array}{c}
x_{1 t} \\
f_{1 t}
\end{array}\right)+\left(\begin{array}{cc}
\psi_{w} & \sigma_{f} \psi_{x f} \\
0 & \sigma_{f}
\end{array}\right)\left(\begin{array}{c}
w_{t+1} \\
w_{t+1}^{f}
\end{array}\right)
$$

where $w_{t+1}$ and $w_{t+1}^{f}$ are uncorrelated innovations. The matrices $\psi_{x}$ and $\psi_{w}$ thus do not involve any direct impact of the dynamics of the belief shock $f_{1 t}$ and the matrix $\psi_{x f}$ captures how the dynamics of $f_{1 t}$ influences the dynamics of endogenous state variables.

Let us further assume that the system (31) represents the equilibrium restrictions of the model except equation (38). In this case, the function $g$ does not directly depend on $f$. Repeating the expansion of the equilibrium conditions from Section B.4 and comparing coefficients on $x_{t-1}, f_{t-1}, w_{t}$ and the constant term yields the set of conditions for matrices $\psi_{x}, \psi_{w}, \psi_{x f}$ and $\psi_{q}$ :

$$
\begin{aligned}
& 0=\left(g_{x+} \psi_{x}+g_{x}\right) \psi_{x}+g_{x-} \\
& 0=\left(g_{x+} \rho_{f} \psi_{x f}-\mathbb{E}\right)+\left(g_{x+} \psi_{x}+g_{x}\right) \psi_{x f} \\
& 0=\left(g_{x+} \psi_{x}+g_{x}\right) \psi_{w}+g_{w} \\
& 0=\left(g_{x+} \psi_{x}+g_{x+}+g_{x}\right) \psi_{q}+g_{q}-\mathbb{E} \bar{f}
\end{aligned}
$$

with

$$
\begin{aligned}
V_{x}= & u_{x}+\beta V_{x} \psi_{x} \\
V_{f}= & u_{f}-\frac{\beta \bar{\theta}}{2}\left(V_{f}^{2} \sigma_{f}^{2}+2 V_{x} \psi_{x f} \sigma_{f}^{2} V_{f}+V_{x}\left(\sigma_{f}^{2} \psi_{x f} \psi_{x f}^{\prime}+\psi_{w} \psi_{w}^{\prime}\right) V_{x}^{\prime}\right) \\
& +\beta\left(V_{f} \rho_{f}+V_{x} \psi_{x f} \rho_{f}\right) \\
\mathbb{E}= & \operatorname{stack}\left\{\sigma^{i}\left[g_{x+} \psi_{x f} \sigma_{f}^{2}\left(V_{f}+V_{x} \psi_{x f}\right)+\left(g_{x+} \psi_{w}+g_{w+}\right) \psi_{w}^{\prime} V_{x}^{\prime}\right]^{i}\right\} \bar{\theta} .
\end{aligned}
$$


This set of equations is the counterpart of equations (34)-(37) and can be solved sequentially. First, notice that equations (40) and (42) can be solved for $\psi_{x}$ and $\psi_{w}$, and these coefficients are not impacted by the dynamics of $f_{t}$. But the equilibrium dynamics of $x_{t}$ is affected by movements in $f_{t}$ through the coefficient $\psi_{x f}$. The coefficient $\rho_{f} \psi_{x f}$ introduces an additional component in the time-varying drift of $x_{t}$, while $\sigma_{f} \psi_{x f}$ is an additional source of volatility arising from the shocks to household's concerns for robustness.

We solve this set of equations by backward induction. First, we use (34), (37) and (44) to find the no-ambiguity solution for $\psi_{x}, \psi_{w}, V_{x}$. Then we postulate that (39) is in fact a time-dependent law of motion

$$
x_{t}=\psi^{t}\left(x_{t-1}, w_{t}, f_{t}\right)
$$

with terminal condition at a distant date $T$

$$
x_{T}=\psi^{T}\left(x_{T-1}, w_{T}, 0\right) .
$$

This corresponds to assuming that starting from date $T$, ambiguity is absent in the model. Plugging this guess to the set of equilibrium conditions, we obtain the set of algebraic equations

$$
\begin{aligned}
0= & \left(g_{x+} \psi_{x f}^{t+1} \rho_{f}-\mathbb{E}^{t+1}\right)+\left(g_{x+} \psi_{x}+g_{x}\right) \psi_{x f}^{t} \\
V_{f}^{t}= & u_{f}-\frac{\beta \bar{\theta}}{2}\left(\left(V_{f}^{t+1} \sigma_{f}\right)^{2}+2 V_{x} \psi_{x f}^{t+1} \sigma_{f}^{2} V_{f}^{t+1}+V_{x}\left(\sigma_{f}^{2} \psi_{x f}^{t+1}\left(\psi_{x f}^{t+1}\right)^{\prime}+\psi_{w} \psi_{w}^{\prime}\right) V_{x}^{\prime}\right) \\
& +\beta \rho_{f}\left(V_{f}^{t+1}+V_{x} \psi_{x f}^{t+1}\right) \\
\mathbb{E}^{t+1}= & {\left[g_{x+} \psi_{x f}^{t+1}\left(V_{f}^{t+1}+V_{x} \psi_{x f}^{t+1}\right) \sigma_{f}^{2}+\left(g_{x+} \psi_{w}+g_{w+}\right) \psi_{w}^{\prime} V_{x}^{\prime}\right] \bar{\theta} . }
\end{aligned}
$$

Equation (47) can then be solved for

$$
\psi_{x f}^{t}=\left(g_{x+} \psi_{x}+g_{x}\right)^{-1}\left(\mathbb{E}^{t+1}-g_{x+} \psi_{x f}^{t+1} \rho_{f}\right)
$$

Iterating backwards on equations (48)-(50) backward until convergence yields the stationary solution of the economy with ambiguity as a long-horizon limit of an economy where ambiguity vanishes at a distant $T$. The system converges as long as its dynamics are stationary under the worst-case model. Once we find the limit $\lim _{t \rightarrow-\infty} \mathbb{E}^{t}=\mathbb{E}$, we can also determine

$$
\psi_{q}=\left(g_{x+} \psi_{x}+g_{x+}+g_{x}\right)^{-1}\left(\mathbb{E} \bar{f}-g_{q}\right) .
$$

\section{B.6 Nonstationary models}

Consider the nonstationary dynamics introduced in Section 4.3. When the period utility function is given by (15), i.e., $u\left(x_{t}\right)=\widehat{u}\left(\widehat{x}_{t}\right)+z_{t}$, then using the guess for the continuation value (16), we can rewrite equation $(25)$ as

$$
\widehat{V}\left(\widehat{x}_{t}\right)=\widehat{u}\left(\widehat{x}_{t}\right)-\frac{\beta}{\theta_{t}} \log E_{t}\left[\exp \left(-\theta_{t}\left(\widehat{V}\left(\widehat{x}_{t+1}\right)+(1-\beta)^{-1} \phi\left(\widehat{x}_{t}, w_{t+1}\right)\right)\right)\right]
$$

with $\widehat{u}\left(\widehat{x}_{t}\right)=\log \widehat{C}\left(x_{t}\right)$. The first-order expansion of $\phi$ yields

$$
\begin{aligned}
\bar{z}_{t+1}-\bar{z}_{t} & =\phi(\bar{x}, 0) \\
z_{1 t+1}-z_{1 t} & =\phi_{q}+\phi_{x} \widehat{x}_{1 t}+\phi_{w} w_{t+1}
\end{aligned}
$$


where $\bar{x}$ is the steady state of $\widehat{x}_{t}$. We can now proceed as in the stationary case except using the expansion of functions $\widehat{u}$ and $\widehat{V}$. We have

$$
\bar{V}=(1-\beta)^{-1}\left[\bar{u}+\beta(1-\beta)^{-1} \phi(\bar{x}, 0)\right]
$$

and

$$
\widehat{V}_{1 t}=V_{x} \widehat{x}_{1 t}+V_{q}
$$

with

$$
\begin{aligned}
& V_{x}=u_{x}+\beta\left[V_{x} \psi_{x}+(1-\beta)^{-1} \phi_{x}\right]-\frac{\beta}{2}\left|V_{x} \psi_{w}+(1-\beta)^{-1} \phi_{w}\right|^{2} \bar{\theta} \\
& V_{q}=u_{q}+\beta\left[V_{q}+V_{x} \psi_{q}+(1-\beta)^{-1} \phi_{q}\right]-\frac{\beta}{2} \bar{\theta} \bar{x}\left|V_{x} \psi_{w}+(1-\beta)^{-1} \phi_{w}\right|^{2} .
\end{aligned}
$$

The zero-th order distortion is consequently given by

$$
m_{0 t+1}=\frac{\exp \left(-\bar{\theta}\left(\bar{x}+\widehat{x}_{1 t}\right)\left(V_{x} \psi_{w}+(1-\beta)^{-1} \phi_{w}\right) w_{t+1}\right)}{E_{t}\left[\exp \left(-\bar{\theta}\left(\bar{x}+\widehat{x}_{1 t}\right)\left(V_{x} \psi_{w}+(1-\beta)^{-1} \phi_{w}\right) w_{t+1}\right)\right]}
$$

so that under the worst-case model,

$$
w_{t+1} \sim N\left(-\bar{\theta}\left(\bar{x}+\widehat{x}_{1 t}\right)\left(V_{x} \psi_{w}+(1-\beta)^{-1} \phi_{w}\right)^{\prime}, I_{k}\right) .
$$

Equation (12) then becomes

$$
\begin{aligned}
\widehat{x}_{1 t+1}= & \psi_{q}-\bar{\theta} \bar{x} \psi_{w}\left(V_{x} \psi_{w}+(1-\beta)^{-1} \phi_{w}\right)^{\prime} \\
& +\left[\psi_{x}-\psi_{w}\left(V_{x} \psi_{w}+(1-\beta)^{-1} \phi_{w}\right)^{\prime} \bar{\theta}\right] \widehat{x}_{1 t}+\psi_{w} \widetilde{w}_{t+1} \\
= & \widetilde{\psi}_{q}+\widetilde{\psi}_{x} x_{1 t}+\psi_{w} \widetilde{w}_{t+1} .
\end{aligned}
$$

We are still solving the set of equations (34)-(36) but now with $V_{x}$ and $\mathbb{E}$ given by

$$
\begin{aligned}
V_{x} & =u_{x}+\beta\left[V_{x} \psi_{x}+(1-\beta)^{-1} \phi_{x}\right]-\frac{\beta}{2}\left|V_{x} \psi_{w}+(1-\beta)^{-1} \phi_{w}\right|^{2} \bar{\theta} \\
\mathbb{E} & =\operatorname{stack}\left\{\sigma_{i}\left[g_{x+} \psi_{w}+g_{w+}\right]^{i}\left(V_{x} \psi_{w}+(1-\beta)^{-1} \phi_{w}\right)^{\prime} \bar{\theta}\right\} .
\end{aligned}
$$

In the special case described in Section B.5, the belief shock $f_{t}$ is modeled as an exogenous $\operatorname{AR}(1)$ process. The first-order dynamics of the stochastic growth rate can be expressed as

$$
z_{1 t+1}-z_{1 t}=\phi_{q}+\phi_{x} \widehat{x}_{1 t}+\phi_{x f} f_{1 t}+\phi_{w} w_{t+1}+\phi_{w f} w_{t+1}^{f} .
$$

The only modifications appearing in the model solution are those related to the continuation value recursion 
and the shock distortion in $\mathbb{E}$. Specifically,

$$
\begin{aligned}
V_{x}= & u_{x}+\beta\left[V_{x} \psi_{x}+(1-\beta)^{-1} \phi_{x}\right] \\
V_{f}= & u_{f}+\beta\left(\rho_{f} V_{f}+\rho_{f} V_{x} \psi_{x f}+(1-\beta)^{-1} \phi_{x f}\right) \\
& -\frac{\beta \bar{\theta}}{2}\left|V_{x} \psi_{w}+(1-\beta)^{-1} \phi_{w}\right|^{2}-\frac{\beta \bar{\theta}}{2}\left|V_{x} \psi_{x f} \sigma_{f}+V_{f} \sigma_{f}+(1-\beta)^{-1} \phi_{w f}\right|^{2} \\
\mathbb{E}= & \operatorname{stack}\left\{\sigma^{i}\left[\left(g_{x+} \psi_{w}+g_{w+}\right)\left(V_{x} \psi_{w}+(1-\beta)^{-1} \phi_{w}\right)^{\prime}\right]^{i}\right\} \bar{\theta} \\
& +\operatorname{stack}\left\{\sigma^{i}\left[g_{x+} \psi_{x f} \sigma_{f}\left(V_{f} \sigma_{f}+V_{x} \psi_{x f} \sigma_{f}+(1-\beta)^{-1} \phi_{w f}\right)\right]^{i}\right\} \bar{\theta}
\end{aligned}
$$

In the recursive form, $V_{f}$ and $\mathbb{E}$ can be solved by iterating on the pair of equations

$$
\begin{aligned}
V_{f}^{t}= & u_{f}+\beta\left(\rho_{f} V_{f}^{t+1}+\rho_{f} V_{x} \psi_{x f}^{t+1}+(1-\beta)^{-1} \phi_{x f}\right) \\
& -\frac{\beta \bar{\theta}}{2}\left|V_{x} \psi_{w}+(1-\beta)^{-1} \phi_{w}\right|^{2}-\frac{\beta \bar{\theta}}{2}\left|V_{x} \psi_{x f}^{t+1} \sigma_{f}+V_{f}^{t+1} \sigma_{f}+(1-\beta)^{-1} \phi_{w f}\right|^{2} \\
\mathbb{E}^{t+1}= & \operatorname{stack}\left\{\sigma^{i}\left[\left(g_{x+} \psi_{w}+g_{w+}\right)\left(V_{x} \psi_{w}+(1-\beta)^{-1} \phi_{w}\right)^{\prime}\right]^{i}\right\} \bar{\theta} \\
& +\operatorname{stack}\left\{\sigma^{i}\left[g_{x+} \psi_{x f}^{t+1} \sigma_{f}\left(V_{f}^{t+1} \sigma_{f}+V_{x} \psi_{x f}^{t+1} \sigma_{f}+(1-\beta)^{-1} \phi_{w f}\right)\right]^{i}\right\} \bar{\theta} .
\end{aligned}
$$

together with equation (50) which remained unchanged.

\section{Data}

Macroeconomic data is collected from the Federal Reserve Bank of St. Louis database (FRED). ${ }^{12}$ Data for the Survey of Professional Forecasters are collected from the Federal Reserve Bank of Philadelphia website. ${ }^{13}$ The data on households' expectations are obtained from the University of Michigan Survey of Consumers. ${ }^{14}$ See Table 4 for details.

For the inflation rate, we record the cross-sectional mean, median and quartile answers. The survey question on unemployment rate only records up/same/down responses. We use the method from Carlson and Parkin (1975) and Mankiw et al. (2003) to fit a time series of normal distributions to these qualitative responses. Let $q_{t}^{u}, q_{t}^{s}$ and $q_{t}^{d}$ be the fractions of survey answers up, same, down, respectively, recorded at time $t$. We assume that these categories are constructed from a continuous cross-sectional distribution of responses with normal density $N\left(\mu_{t}, \sigma_{t}^{2}\right)$. In particular, there exists a response threshold $a$ such that an answer on the interval $[-a, a]$ is recorded as 'same'. This implies

$$
q_{t}^{d}=\Phi\left(\frac{-a-\mu_{t}}{\sigma_{t}}\right) \quad q_{t}^{u}=1-\Phi\left(\frac{a-\mu_{t}}{\sigma_{t}}\right)
$$

and thus

$$
-a-\mu_{t}=\sigma_{t} \Phi^{-1}\left(q_{t}^{d}\right) \quad a-\mu_{t}=\sigma_{t} \Phi^{-1}\left(1-q_{t}^{u}\right)
$$

\footnotetext{
${ }^{12}$ https://research.stlouisfed.org/fred2/

${ }^{13}$ https://www.philadelphiafed.org/research-and-data/real-time-center/survey-of-professional-forecasters/

${ }^{14}$ http://www.sca.isr.umich.edu/ See also Thomas (1999) for details on the survey methodology.
} 


\begin{tabular}{ll} 
Macroeconomic & variables (FRED) \\
\hline$\pi_{t}$ & Consumer price index for all urban consumers: All items, seasonally adjusted \\
& (CPIAUCSL). Quarterly logarithmic growth rate, last month to last month of quarter. \\
$u_{t}$ & Civilian unemployment rate, quarterly, seasonally adjusted (UNRATE). \\
$\log \left(\mathcal{Y}_{t} / \mathcal{Y}_{t-1}\right)$ & Real gross domestic product, quarterly, seasonally adjusted annual rate (GDPC96). \\
& Quarterly logarithmic growth rate. \\
$i_{t}$ & Effective Federal Funds Rate (FEDFUNDS). Quarterly averages.
\end{tabular}

\section{Households' expectations (Michigan Survey)}

\begin{tabular}{|c|c|}
\hline$\widetilde{E}_{t}\left[\sum_{j=1}^{4} \pi_{t+j}\right]$ & $\begin{array}{l}\text { Expected change in prices during the next year (Table } 32 \text {, variable PX1), mean response } \\
\text { and quartiles of the cross-sectional distribution of individual answers. As response for } \\
\text { quarter } t \text {, we use the survey round from the first month of quarter } t+1 \text {. Questions: } \\
\text { "During the next } 12 \text { months, do you think that prices in general will go up, or go down, } \\
\text { or stay where they are now?" and "By about what percent do you expect prices to go } \\
\text { up, on the average, during the next } 12 \text { months?" }\end{array}$ \\
\hline$\widetilde{E}_{t}\left[u_{t+4}\right]$ & $\begin{array}{l}\text { Expected unemployment rate during next year (Table } 30 \text {, variable UMEX), construc- } \\
\text { tion of mean response detailed in the main text. As response for quarter } t \text {, we use the } \\
\text { survey round from the first month of quarter } t+1 \text {. Question: "How about people out } \\
\text { of work during the coming } 12 \text { months - do you think there will be more unemployment } \\
\text { than now, about the same, or less?" }\end{array}$ \\
\hline$\widetilde{E}_{t}\left[\log \left(\mathcal{Y}_{t+4} / \mathcal{Y}_{t}\right)\right]$ & $\begin{array}{l}\text { Index of current conditions (Table } 5 \text {, variable ICC) and index of consumer expectations } \\
\text { (Table } 5 \text {, variable ICE) used as a proxy for the GDP growth forecast. Construction } \\
\text { described in the main text. As response for quarter } t \text {, we use the survey round from } \\
\text { the first month of quarter } t+1 \text {. }\end{array}$ \\
\hline
\end{tabular}

\section{Survey of Professional Forecasters}

\begin{tabular}{|c|c|}
\hline \begin{tabular}{l|l}
$E_{t}$ & $\left.\sum_{j=1}^{4} \pi_{t+j}\right]$
\end{tabular} & $\begin{array}{l}\text { Forecasted CPI inflation rate, seasonally adjusted (CPI). Forecast at time } t \text { is con- } \\
\text { structed as the mean survey forecast made in second month of quarter } t+1 \text {, for CPI } \\
\text { inflation rate between quarters } t \text { and } t+4 \text {. }\end{array}$ \\
\hline$E_{t}\left[u_{t+4}\right]$ & $\begin{array}{l}\text { Forecasted unemployment rate, seasonally adjusted (UNEMP). Forecast at time } t \text { is } \\
\text { constructed as the mean survey forecast made in second month of quarter } t+1 \text {, for the } \\
\text { average unemployment rate in quarter } t+4 \text {. }\end{array}$ \\
\hline$E_{t}\left[\log \left(\mathcal{Y}_{t+4} / \mathcal{Y}_{t}\right)\right]$ & $\begin{array}{l}\text { Forecasted GDP growth rate, logarithmic annual growth rate (RGDP). Forecast at } \\
\text { time } t \text { is constructed as the mean survey forecast made in second month of quarter }\end{array}$ \\
\hline
\end{tabular}

Table 4: Data definitions for the macroeconomic and survey variables. 
and therefore

$$
\begin{aligned}
\sigma_{t} & =\frac{2 a}{\Phi^{-1}\left(1-q_{t}^{u}\right)-\Phi^{-1}\left(q_{t}^{d}\right)} \\
\mu_{t} & =a-\sigma_{t} \Phi^{-1}\left(1-q_{t}^{u}\right)
\end{aligned}
$$

The constant $a$ is then determined so that the time-series average of the cross-sectional dispersions $\sigma_{t}$ divided by the observed average cross-sectional dispersion for the SPF forecast corresponds to the analogous ratio for the inflation responses, for which we have dispersion data readily available.

To construct a proxy for the GDP growth survey response, we regress the realized 4-quarter GDP growth $\log \left(\mathcal{Y}_{t} / \mathcal{Y}_{t-4}\right)$ on the index of current conditions $I C C_{t}$ (see Table 4)

$$
\log \left(\mathcal{Y}_{t} / \mathcal{Y}_{t-4}\right)=\beta_{0}+\beta_{1} I C C_{t}+\varepsilon_{t}
$$

We then use the estimated regression coefficients to construct a proxy for the household's subjective GDP growth forecast using the index of consumer expectations $I C E_{t}$

$$
\widetilde{E}_{t}\left[\log \left(\mathcal{Y}_{t+4} / \mathcal{Y}_{t}\right)\right]=\widehat{\beta}_{0}+\widehat{\beta}_{1} I C E_{t}
$$

We construct the time series of belief wedges for the period 1982Q2-2015Q4 by taking the difference between the household and SPF forecasts.

\section{C.1 Details on the belief wedges}

Table 5 contains time-series characteristics of the belief wedges. Table 6 shows the conditional time-series averages of the households' forecasts for different demographic groups.

\section{Estimation of the one-factor model}

Recall that we estimate the model

$$
\begin{aligned}
\left(\begin{array}{c}
y_{t+1} \\
f_{t+1}
\end{array}\right) & =\left(\begin{array}{cc}
\psi_{y} & \psi_{y f} \rho_{f} \\
0 & \rho_{f}
\end{array}\right)\left(\begin{array}{c}
y_{t} \\
f_{t}
\end{array}\right)+\left(\begin{array}{cc}
\psi_{y w} & \psi_{y f} \sigma_{f} \\
0 & \sigma_{f}
\end{array}\right)\left(\begin{array}{c}
w_{t+1}^{y} \\
w_{t+1}^{f}
\end{array}\right) \\
\bar{\Delta}_{t+1}^{(4)} & =\psi_{\Delta f} f_{t+1}+\sigma_{\Delta} \varepsilon_{t+1}^{\Delta}
\end{aligned}
$$

We estimate the model using a Metropolis-Hastings algorithm. We take five chains with different initial draws and make 20,000 draws in each chain. The first 10,000 draws of each chain are dropped.

The priors and posterior parameter estimates are reported in Table 7. The Inverse-Gamma priors on the standard deviations shrink the estimates away from zero in order to prevent overfitting. The priors for the measurement error standard deviations have means that are scaled by the standard deviations of the wedges. The variance decomposition at the estimated mode of the parameters is reported in Table 8 . 


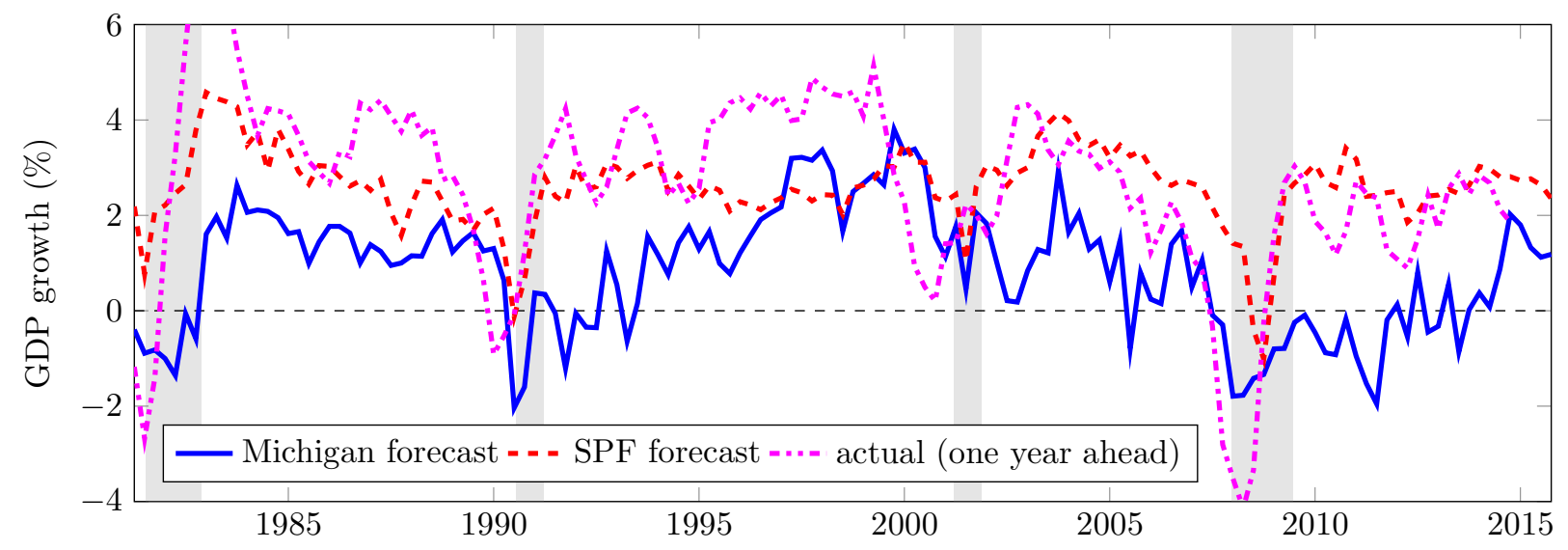

Figure 9: One-year GDP growth forecasts from the Survey of Professional Forecasters and Michigan Survey, and the realized GDP growth (shifted one year ahead). The Michigan Survey forecast is constructed using a projection on the Index of Consumer Expectations, see text in the appendix for details. NBER recessions shaded.

\begin{tabular}{l|rr|rrrrr} 
& & & \multicolumn{5}{|c}{ correlation matrix } \\
& mean & std & $\Delta_{t}^{(4)}(\pi)$ & $\Delta_{t}^{(4)}(u)$ & $\Delta_{t}^{(4)}(d \mathcal{Y})$ & $\log \left(\mathcal{Y}_{t} / \mathcal{Y}_{t-4}\right)$ & $\log \left(\mathcal{Y}_{t} / \overline{\mathcal{Y}}_{t}\right)$ \\
\hline Inflation wedge $\Delta_{t}^{(4)}(\pi)$ & 1.01 & 0.79 & 1.00 & 0.04 & -0.32 & -0.26 & -0.31 \\
Unemployment wedge $\Delta_{t}^{(4)}(u)$ & 0.55 & 0.39 & & 1.00 & -0.63 & -0.46 & -0.34 \\
GDP growth wedge $\Delta_{t}^{(4)}(d \mathcal{Y})$ & -1.73 & 1.18 & & & 1.00 & 0.41 & 0.68 \\
GDP growth $\log \left(\mathcal{Y}_{t} / \mathcal{Y}_{t-4}\right)$ & 2.66 & 2.02 & & & & 1.00 & 0.60 \\
Output gap $\log \left(\mathcal{Y}_{t} / \mathcal{\mathcal { Y }}_{t}\right)$ & -1.87 & 2.30 & & & & & 1.00
\end{tabular}

Table 5: Time-series and business cycle statistics for the belief wedges. All values are in percent, time period 1981Q2-2015Q4.

\begin{tabular}{r|rrrrrrrrrrrr} 
& actual & SPF & all & $18-34$ & $35-44$ & $45-54$ & $55-64$ & $65+$ & W & NC & NE & S \\
\hline$\pi$ & 2.82 & 3.01 & 4.05 & 4.14 & 4.07 & 4.02 & 3.86 & 3.96 & 3.98 & 4.00 & 4.00 & 4.18 \\
$u$ & 6.34 & 6.34 & 6.88 & 6.74 & 6.90 & 6.97 & 6.98 & 6.88 & 6.86 & 6.87 & 6.93 & 6.86 \\
& & & & & & & & & & & & \\
& & & male & female & bottom & 2nd Q & 3 rd Q & top & HS & SC & COL & GS \\
\hline$\pi$ & & & 3.47 & 4.56 & 4.99 & 4.28 & 3.77 & 3.27 & 4.58 & 3.98 & 3.57 & 3.46 \\
$u$ & & & 6.79 & 6.95 & 7.03 & 6.90 & 6.85 & 6.73 & 6.97 & 6.88 & 6.77 & 6.79
\end{tabular}

Table 6: Demographic characteristics of households' expectations on inflation and unemployment rate. Time-series averages, all values are annualized and in percent, time period 1981Q2-2015Q4. Actual: actual average inflation and unemployment rate; $S P F$ : average SPF forecast; all: average household forecast; 18-34 etc: age groups; $W$ : West region; $N C$ : North-Central; $N E$ : North-East; $S$ : South; bottom, 2nd $Q$, 3rd $Q$, top: income quartiles; $H S$ : high-school education; $S C$ : some college; $C O L$ : college degree; $G S$ : graduate studies. 


\begin{tabular}{|c|c|c|}
\hline Parameter & $\begin{array}{c}\text { Prior } \\
\text { D (Mean,Std) }\end{array}$ & $\begin{array}{c}\text { Posterior } \\
\text { Mean (90\% HPD) }\end{array}$ \\
\hline \multicolumn{3}{|l|}{ VAR coefficients } \\
\hline $\begin{array}{l}\psi_{y, i i} \\
\psi_{y, i j}, i \neq j \\
100 \psi_{w, i i} \\
\psi_{w, i j} / \psi_{w, j j}, i \neq j \\
\psi_{y f, d \mathcal{Y}} \\
\psi_{y f, u} \\
\psi_{y f, \pi} \\
\psi_{y f, R}\end{array}$ & $\begin{array}{c}\mathcal{B}(0.7,0.2) \\
N(0,2) \\
\mathcal{I} \mathcal{G}(1,0.5) \\
N(0,2) \\
N(0,1) \\
N(0,1) \\
N(0,1) \\
N(0,1)\end{array}$ & $\begin{array}{c}-0.39(-0.61,-0.16) \\
0.10(0.01,0.19) \\
0.08(-0.00,0.16) \\
-0.06(-0.12,0.00)\end{array}$ \\
\hline \multicolumn{3}{|l|}{ Factor coefficients } \\
\hline $\begin{array}{l}\rho_{f} \\
100 \sigma_{f} \\
H_{u} \\
H_{\pi} \\
H_{R} \\
H_{f}\end{array}$ & $\begin{array}{c}\mathcal{B}(0.5,0.1) \\
\mathcal{I} \mathcal{G}(0.5,0.2) \\
N(0,1) \\
N(0,1) \\
N(0,1) \\
N(0,1)\end{array}$ & $\begin{array}{c}0.80(0.73,0.88) \\
0.47(0.28,0.63) \\
0.51(0.09,0.88) \\
0.56(0.20,0.92) \\
0.14(-1.62,1.94) \\
-0.72(-1.18,-0.26)\end{array}$ \\
\hline \multicolumn{3}{|l|}{ Measurement errors } \\
\hline $100 \sigma_{\Delta, d \mathcal{Y}} \quad$ GDP growth wedge & $\overline{\mathcal{I} \mathcal{G}(0.6,0.3)}$ & $0.16(0.14,0.17)$ \\
\hline Unemployment wedge & $\mathcal{I G}(0.8,0.4)$ & $0.33(0.30,0.37)$ \\
\hline $100 \sigma_{\Delta, \pi} \quad$ Inflation wedge & $\mathcal{I} \mathcal{G}(0.4,0.2)$ & $0.19(0.17,0.21)$ \\
\hline
\end{tabular}

Table 7: Factor model prior and posterior estimates. The priors $\mathcal{I} \mathcal{G}(\mu, \sigma)$ and $\mathcal{B}(\mu, \sigma)$ denote InverseGamma and Beta distributions with mean $\mu$ and standard deviation $\sigma$.

\begin{tabular}{ll|rrrrrr} 
Variable & & $w^{d \mathcal{Y}}$ & $w^{u}$ & $w^{\pi}$ & $w^{R}$ & $w^{f}$ & $\begin{array}{r}\text { meas. } \\
\text { error }\end{array}$ \\
\hline $\log \left(\mathcal{Y}_{t} / \mathcal{Y}_{t-1}\right)$ & GDP growth & 56.0 & 27.2 & 1.8 & 2.0 & 13.1 & - \\
$u_{t}$ & Unemployment & 5.4 & 31.0 & 18.3 & 24.3 & 21.0 & - \\
$\pi_{t}$ & Inflation & 3.3 & 17.2 & 70.0 & 5.5 & 4.0 & - \\
$R_{t}$ & Nominal interest & 4.4 & 19.3 & 36.3 & 34.7 & 5.3 & - \\
$\bar{\Delta}_{t}^{(4)}(d \mathcal{Y})$ & GDP growth wedge & - & - & - & - & 67.5 & 32.5 \\
$\bar{\Delta}_{t}^{(4)}(u)$ & Unemployment wedge & - & - & - & - & 23.2 & 76.8 \\
$\bar{\Delta}_{t}^{(4)}(\pi)$ & Inflation wedge & - & - & - & - & 9.7 & 90.3
\end{tabular}

Table 8: Factor model variance decomposition at the posterior modes. All values are in percent. 


\section{E Details on the New-Keynesian model}

\section{E.1 Alternating offer bargaining}

As in Hall and Milgrom (2008) and Christiano et al. (2015), we assume that wages are determined by the alternating offer bargaining protocol of Rubinstein (1982) and Binmore et al. (1986).

At the start of period $t, l_{t}$ matches are determined. Each worker then engages in bilateral bargaining with a wholesaler firm over the current wage rate $\xi_{t}$ that is conditional on all other period $t$ matches as well as beliefs about future wage bargains.

The bargaining takes place across $\tau$ subperiods within the period, where $\tau$ is even. Conditional on all previous offers having been rejected, the firm makes a wage offer every odd subperiod, while the worker makes a wage offer every even subperiod. The recipient can accept or reject an offer. If the recipient rejects an offer, she can end negotiations or plan to make a counteroffer in the next subperiod. In the latter case, the bargaining breaks down with probability $\delta$. We assume that when indifferent between accepting and rejecting an offer, an agent accepts it.

Christiano et al. (2015) show that the solution to the bargaining problem yields the condition

$$
J_{t}=\beta_{1}\left(W_{t}-U_{t}\right)-\beta_{2} \gamma_{t}+\beta_{3}\left(\vartheta_{t}-D_{t}\right)
$$

where $\beta_{i}=\alpha_{i+1} / \alpha_{i}$, with $\alpha_{i}$ defined as follows:

$$
\begin{aligned}
\alpha_{1} & =1-\delta+(1-\delta)^{\tau} \\
\alpha_{2} & =1-(1-\delta)^{\tau} \\
\alpha_{3} & =\alpha_{2} \frac{1-\delta}{\delta}-\alpha_{1} \\
\alpha_{4} & =\frac{1-\delta}{2-\delta} \frac{\alpha_{2}}{\tau}+1-\alpha_{2} .
\end{aligned}
$$

\section{E.2 Growth rate and functional forms}

The model has two sources of growth - neutral and investment-specific technological progress. For a balanced growth path in the nonstochastic steady state, we require that the elements $\left\{\phi_{t}, s_{t}, \kappa_{t}, \gamma_{t}, G_{t}, D_{t}\right\}$ grow in the long run at the same rate

$$
\Phi_{t}=\Psi_{t}^{\frac{\alpha}{1-\alpha}} A_{t}
$$

which corresponds to the stochastic aggregate growth rate of the economy. We thus set

$$
\left(\phi_{t}, s_{t}, \kappa_{t}, \gamma_{t}, G_{t}, D_{t}\right)^{\prime}=(\phi, s, \kappa, \gamma, G, D)^{\prime} \Omega_{t}
$$

where $\Omega_{t}$ is defined as

$$
\Omega_{t}=\Phi_{t-1}^{0.05} \Omega_{t-1}^{0.95}
$$

The capacity utilization cost is set to

$$
a_{u}\left(u_{t}^{K}\right)=\frac{0.11}{2} \varphi\left(u_{t}^{K}\right)^{2}+0.89 \varphi u_{t}^{K}+\varphi\left(\frac{0.11}{2}-1\right)
$$


where $\varphi$ is chosen so that the steady state value of $u_{t}^{K}$ is one. Finally, investment adjustment costs are given by

$$
a_{I}\left(\frac{I_{t}}{I_{t-1}}\right)=\frac{1}{2}\left[\exp \left(\sqrt{15.7}\left(\frac{I_{t}}{I_{t-1}}-\mu \times \mu_{\Psi}\right)\right)+\exp \left(-\sqrt{15.7}\left(\frac{I_{t}}{I_{t-1}}-\mu \times \mu_{\Psi}\right)\right)\right]-1
$$

where $\mu$ and $\mu_{\Psi}$ denote the unconditional growth rates of $\Phi_{t}$ and $\Psi_{t}$ respectively. The functional forms and parameter values for $\Omega_{t}, a_{u}$ and $a_{I}$ are taken from Christiano et al. (2015).

\section{F Detection error probabilities}

Let $\left\{x_{t}\right\}_{t=1}^{T}$ be a sample of data simulated from the linear approximation of the benchmark model (10). The $\log$-likelihood of the data under the benchmark model is, up to an additive constant ${ }^{15}$

$$
\begin{aligned}
\log L_{B}^{T} & =-\frac{1}{2} \sum_{t=0}^{T-1}\left(x_{t+1}-\psi_{q}-\psi_{x} x_{t}\right)^{\prime}\left(\psi_{w} \psi_{w}^{\prime}\right)^{-1}\left(x_{t+1}-\psi_{q}-\psi_{x} x_{t}\right)= \\
& =-\frac{1}{2} \sum_{t=0}^{T-1} w_{t+1}^{\prime} w_{t+1} .
\end{aligned}
$$

Under the worst-case model (12), the data follows

$$
\begin{aligned}
\log L_{W}^{T} & =-\frac{1}{2} \sum_{t=0}^{T-1}\left(x_{t+1}-\widetilde{\psi}_{q}-\widetilde{\psi}_{x} x_{t}\right)^{\prime}\left(\psi_{w} \psi_{w}^{\prime}\right)^{-1}\left(x_{t+1}-\widetilde{\psi}_{q}-\widetilde{\psi}_{x} x_{t}\right) \\
& =-\frac{1}{2} \sum_{t=0}^{T-1}\left[w_{t+1}+\theta_{t}\left(V_{x} \psi_{w}+(1-\beta)^{-1} \phi_{w}\right)^{\prime}\right]^{\prime}\left[w_{t+1}+\theta_{t}\left(V_{x} \psi_{w}+(1-\beta)^{-1} \phi_{w}\right)^{\prime}\right] \\
& =-\frac{1}{2} \sum_{t=0}^{T-1} \widetilde{w}_{t+1}^{\prime} \widetilde{w}_{t+1}
\end{aligned}
$$

where the second line follows from the distribution of $w_{t+1}$ under the nonstationary version of the worst-case model (51), and $\theta_{t}=\bar{\theta}\left(\bar{x}+\widehat{x}_{t}\right)$. Simulating $N$ samples from the law of motion for the benchmark model, we compute the classification error $p_{B}^{T}$ as the fraction of samples for which $\log L_{W}^{T}>\log L_{B}^{T}$, and revert the role of the two models to compute $p_{W}^{T}$.

\section{G Robustness checks}

In this appendix, we show the robustness of our findings for two alternative definitions of belief wedges:

(i) Median: We construct the belief wedge for the inflation forecast using the median (instead of mean) responses in the Michigan Survey and SPF.

(ii) Model-implied: We use the forecasts from the Michigan Survey and construct the wedges relative to the forecast implied by the model (VAR forecast for the factor model and the forecast generated by the structural model at the modal parameters, respectively).

We re-estimate both the factor model from Section 3 and the structural model from Section 5 using these alternative definitions. For the model-implied specification, the observation equations for wedges are replaced

\footnotetext{
${ }^{15}$ For notational simplicity, we assume that data $x_{t}$ is a subset of the observable variables that makes the law of motion (10) stochastically non-singular, so that $\psi_{w} \psi_{w}^{\prime}$ is invertible.
} 
with

$$
\widetilde{E}_{t} \zeta_{t+\tau}=E_{t} \zeta_{t+\tau}+\bar{\Delta}_{t}^{(\tau)}+\sigma_{t}^{\Delta} \varepsilon_{t}
$$

where $\widetilde{E}_{t} \zeta_{t+\tau}$ are the observed data on household forecasts, $E_{t} \zeta_{t+\tau}$ is the model-based forecast, $\bar{\Delta}_{t}^{(\tau)}$ is the implied belief wedge, and $\varepsilon_{t}$ are the measurement errors.

The results for the factor model are summarized in Tables 9 and 10 and the corresponding impulse responses are in Figure 10. The estimation using median inflation forecasts yields essentially unchanged results. The results for the estimation that computes belief wedges relative to model-implied forecasts show qualitatively very similar responses but the relative magnitudes of the belief wedge responses are smaller for the GDP growth and the unemployment rate. Table 10 reveals that the belief factor in the specification with model-implied wedges is able to explain a large share of variation in the household forecasts $(63 \%, 29 \%$ and $13 \%$, respectively), but is significantly less successful in explaining the variation in the constructed wedges $(35 \%, 10 \%$ and $21 \%)$. This confirms our view that it is appropriate to construct belief wedges directly from observable data on household and SPF forecasts, rather than relying on household forecast data relative to model-implied forecasts.

For the structural model, the estimation results and the variance decomposition are summarized in Tables 11 and 12. The corresponding impulse responses are in Figure 11. These results corroborate those from the robustness checks in the factor model and we see that the results are essentially unchanged across the different ways of defining the wedges.

In Figure 12, we plot the time-series paths for the belief wedges and the implied factor across the alternative measurement choices in the structural model. The model-implied wedges (dotted red) are constructed using the smoothed shocks at the posterior modes. The last panel in Figure 12 plots the standardized time series for the extracted factor in all the three cases. We see that the wedges are highly correlated and the common component has very similar dynamics over the business cycle, confirming the results from the baseline specification. 
GDP growth

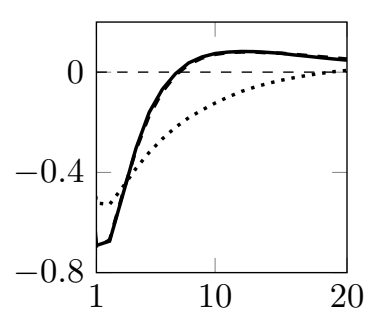

GDP growth wedge

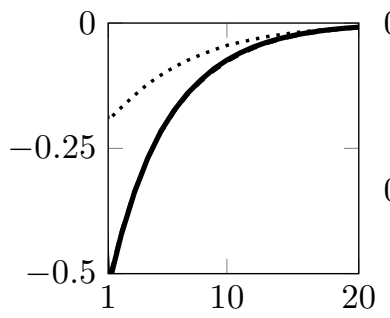

unemployment

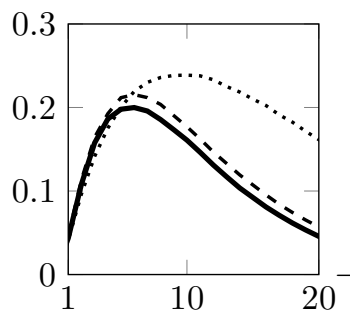

unemp. wedge

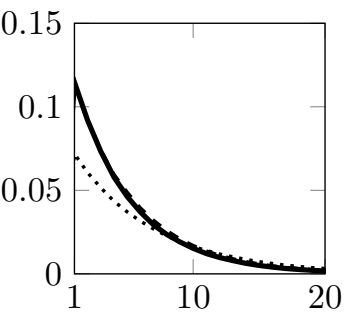

inflation

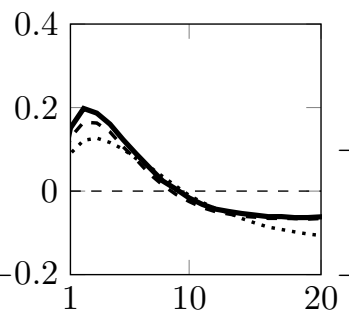

inflation wedge

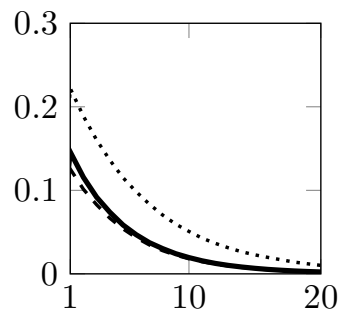

interest rate

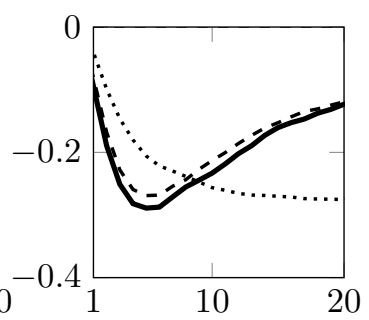

belief factor

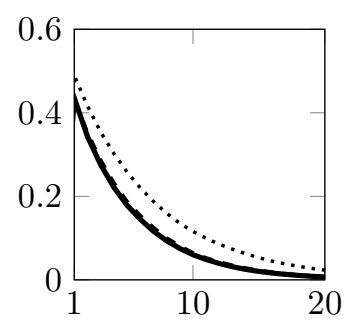

Figure 10: Impulse response functions to the belief shock $w^{f}$ in the factor model. GDP growth, inflation rate and interest rate in annualized percent, and unemployment rate in percentage points. The solid lines use wedges defined as the difference in the mean responses of the household and professional forecasters, the dashed lines use the wedges computed with the median household survey responses, and the dotted lines use the wedges relative to the model-implied forecasts. Horizontal axis in quarters.

\begin{tabular}{l|rrr} 
& & & \\
Parameter & base & med & mod \\
\hline & & & \\
VAR coefficients & & & \\
\hline$\psi_{y f, d \mathcal{Y}}$ & -0.39 & -0.41 & -0.27 \\
$\psi_{y f, u}$ & 0.10 & 0.11 & 0.08 \\
$\psi_{y f, \pi}$ & 0.08 & 0.08 & 0.05 \\
$\psi_{y f, R}$ & -0.06 & -0.05 & -0.02 \\
Factor coefficients & & & \\
\hline$\rho_{f}$ & & & \\
$100 \sigma_{f}$ & 0.80 & 0.81 & 0.85 \\
$H_{u}$ & 0.47 & 0.56 & 0.51 \\
$H_{\pi}$ & 0.51 & -0.46 & 0.80 \\
$H_{R}$ & 0.14 & 0.53 & 0.29 \\
$H_{f}$ & 0.56 & 0.22 & 1.03 \\
& -0.72 & -0.72 & -1.68 \\
Measurement errors & & & \\
\hline $100 \sigma_{\Delta, d \mathcal{Y}} \quad$ GDP growth wedge & 0.16 & 0.16 & 0.12 \\
$100 \sigma_{\Delta, u} \quad$ Unemployment wedge & 0.33 & 0.33 & 0.38 \\
$100 \sigma_{\Delta, \pi} \quad$ Inflation wedge & 0.19 & 0.22 & 0.22
\end{tabular}

Table 9: Factor model parameters estimated using alternative measures of wedges. The column 'base' uses wedges defined as the difference in the mean responses of the household and professional forecasters, the column 'med' computes the wedges using the median household survey responses, and the column 'mod' computes the wedges relative to the model-implied forecasts. 


\begin{tabular}{ll|rrr|rrr|rrr} 
Variable & & \multicolumn{4}{|c|}{$w^{d \mathcal{Y}}$} & \multicolumn{4}{|c}{$w^{u}$} & \multicolumn{3}{|c}{$w^{\pi}$} \\
& & base & med & mod & base & med & mod & base & med & mod \\
\hline $\log \left(\mathcal{Y}_{t} / \mathcal{Y}_{t-1}\right)$ & GDP growth & 56.0 & 56.7 & 62.7 & 27.2 & 26.9 & 22.3 & 1.8 & 1.7 & 1.8 \\
$u_{t}$ & Unemployment & 5.4 & 5.3 & 4.0 & 31.0 & 30.5 & 23.9 & 18.3 & 18.6 & 17.3 \\
$\pi_{t}$ & Inflation & 3.3 & 3.1 & 3.5 & 17.2 & 16.7 & 17.7 & 70.0 & 70.8 & 62.9 \\
$R_{t}$ & Nominal interest & 4.4 & 4.2 & 6.1 & 19.3 & 18.8 & 27.2 & 36.3 & 36.7 & 18.5 \\
$\bar{\Delta}_{t}^{(4)}(d \mathcal{Y})$ & GDP growth wedge & - & - & - & - & - & - & - & - & - \\
$\bar{\Delta}_{t}^{(4)}(u)$ & Unemp. wedge & - & - & - & - & - & - & - & - & - \\
$\bar{\Delta}_{t}^{(4)}(\pi)$ & Inflation wedge & - & - & - & - & - & - & - & - & - \\
$\widetilde{E}_{t} \log \left(\mathcal{Y}_{t+4} / \mathcal{Y}_{t}\right)$ & GDP growth forecast & - & - & 2.2 & - & - & 7.7 & - & - & 8.6 \\
$\widetilde{E}_{t} u_{t+4}$ & Unemp. forecast & - & - & 3.3 & - & - & 17.6 & - & - & 17.0 \\
$\widetilde{E}_{t} \pi_{t+4}$ & Inflation forecast & - & - & 4.2 & - & - & 20.4 & - & - & 36.9
\end{tabular}

\begin{tabular}{ll|rrr|rrr|rrr} 
Variable & & \multicolumn{4}{|c|}{$w^{R}$} & \multicolumn{4}{|c|}{$w^{f}$} & \multicolumn{3}{|c}{ meas. error } \\
& & base & med & mod & base & med & mod & base & med & mod \\
\hline $\log \left(\mathcal{Y}_{t} / \mathcal{Y}_{t-1}\right)$ & GDP growth & 2.0 & 1.9 & 1.6 & 13.1 & 12.8 & 11.6 & - & - & - \\
$u_{t}$ & Unemployment & 24.3 & 24.3 & 29.9 & 21.0 & 21.3 & 25.0 & - & - & - \\
$\pi_{t}$ & Inflation & 5.5 & 5.6 & 8.8 & 4.0 & 3.8 & 7.1 & - & - & - \\
$R_{t}$ & Nominal interest & 34.7 & 34.6 & 32.1 & 5.3 & 5.7 & 16.0 & - & - & - \\
$\bar{\Delta}_{t}^{(4)}(d \mathcal{Y})$ & GDP growth wedge & - & - & - & 67.5 & 67.0 & 35.1 & 32.5 & 33.0 & 64.9 \\
$\bar{\Delta}_{t}^{(4)}(u)$ & Unemp. wedge & - & - & - & 23.2 & 24.6 & 10.5 & 76.8 & 75.4 & 89.5 \\
$\bar{\Delta}_{t}^{(4)}(\pi)$ & Inflation wedge & - & - & - & 9.7 & 5.8 & 20.7 & 90.3 & 94.2 & 79.3 \\
$\widetilde{E}_{t} \log \left(\mathcal{Y}_{t+4} / \mathcal{Y}_{t}\right)$ & GDP growth forecast & - & - & 7.8 & - & - & 63.2 & - & - & 10.6 \\
$\widetilde{E}_{t} u_{t+4}$ & Unemp. forecast & - & - & 29.4 & - & - & 29.0 & - & - & 3.6 \\
$\widetilde{E}_{t} \pi_{t+4}$ & Inflation forecast & - & - & 10.6 & - & - & 12.7 & - & - & 15.2
\end{tabular}

Table 10: Factor model variance decomposition using estimates from alternative measures of wedges. The column 'base' uses wedges defined as the difference in the mean responses of the household and professional forecasters, the column 'med' computes the wedges using the median household survey responses, and the column 'mod' computes the wedges relative to the model-implied forecasts. 

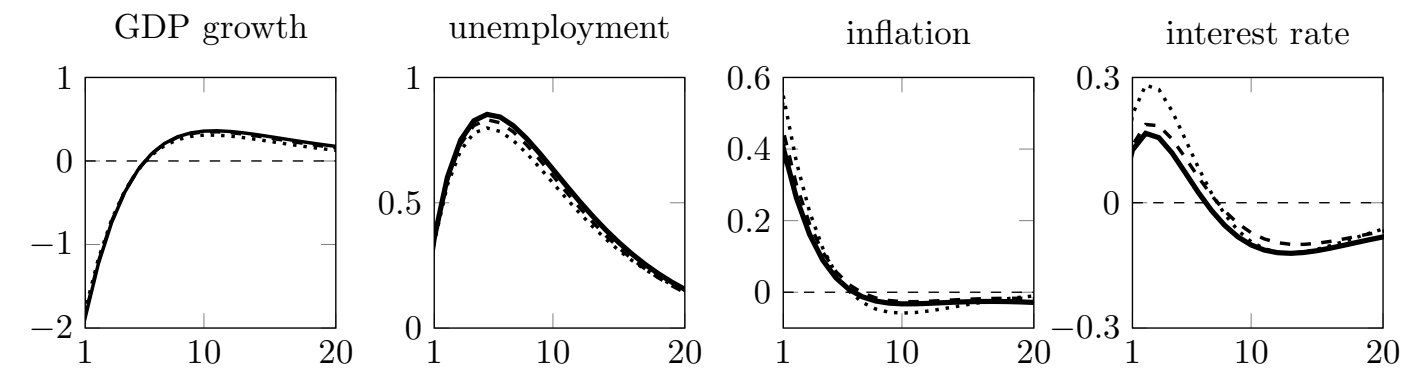

GDP growth wedge
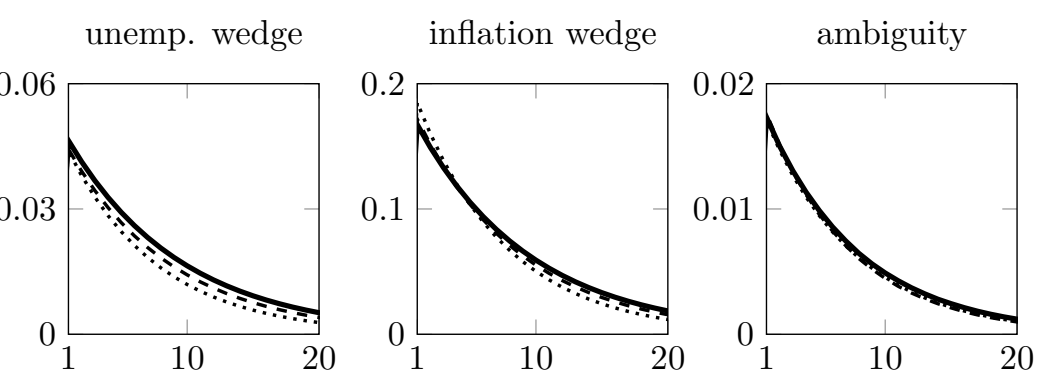

Figure 11: Impulse response functions to the ambiguity shock $w^{f}$ in the structural model. GDP growth, inflation rate and interest rate in annualized percent, and unemployment rate in percentage points. The solid lines use wedges defined as the difference in the mean responses of the household and professional forecasters, the dashed lines use the wedges computed with the median household survey responses, and the dotted lines use the wedges relative to the model-implied forecasts. Horizontal axis in quarters.

\begin{tabular}{|c|c|c|c|c|}
\hline \multicolumn{2}{|c|}{ Parameter } & base & med & $\bmod$ \\
\hline \multicolumn{5}{|c|}{ Monetary policy rule } \\
\hline$\rho_{R}$ & Smoothing & 0.79 & 0.79 & 0.72 \\
\hline$r_{\pi}$ & Inflation & 1.67 & 1.68 & 1.50 \\
\hline$r_{y}$ & Output & 0.03 & 0.03 & 0.03 \\
\hline \multicolumn{5}{|c|}{ Shock processes } \\
\hline & $\mathrm{AR}(1)$ ambiguity & 0.87 & 0.86 & 0.86 \\
\hline$\sigma_{f}$ & Std. dev. ambiguity shock & 0.02 & 0.02 & 0.02 \\
\hline $100 \sigma_{A}$ & Std. dev. neutral tech. shock & 0.86 & 0.86 & 0.86 \\
\hline$\rho_{\Psi}$ & $\mathrm{AR}(1)$ invest. tech. & 0.29 & 0.29 & 0.25 \\
\hline $100 \sigma_{\Psi}$ & Std. dev. invest. tech. shock & 2.88 & 2.97 & 2.72 \\
\hline $100 \sigma_{R}$ & Std. dev. monetary policy shock & 0.24 & 0.24 & 0.26 \\
\hline \multicolumn{5}{|c|}{ Measurement errors } \\
\hline$\overline{100 \sigma_{\Delta, d \mathcal{Y}}}$ & GDP growth wedge & 0.40 & 0.40 & 0.55 \\
\hline $100 \sigma_{\Delta, u}$ & Unemployment wedge & 0.17 & 0.17 & 0.29 \\
\hline $100 \sigma_{\Delta, \pi}$ & Inflation wedge & 0.29 & 0.29 & 0.41 \\
\hline
\end{tabular}

Table 11: Structural model parameters estimated using alternative measures of wedges. The column 'base' uses wedges defined as the difference in the mean responses of the household and professional forecasters, the column 'med' computes the wedges using the median household survey responses, and the column 'mod' computes the wedges relative to the model-implied forecasts. 


\begin{tabular}{ll|rrr|rrr|rrr} 
Variable & & \multicolumn{4}{|c|}{$w^{A}$} & \multicolumn{4}{|c}{$w^{\Psi}$} & \multicolumn{3}{|c}{$w^{R}$} \\
& & base & med & mod & base & med & mod & base & med & mod \\
\hline $\log \left(\mathcal{Y}_{t} / \mathcal{Y}_{t-1}\right)$ & GDP growth & 38.4 & 37.9 & 43.2 & 36.1 & 37.7 & 31.2 & 0.8 & 0.9 & 0.6 \\
$u_{t}$ & Unemployment & 41.1 & 40.2 & 46.2 & 45.0 & 47.1 & 39.6 & 0.4 & 0.4 & 0.2 \\
$\pi_{t}$ & Inflation & 53.2 & 52.1 & 52.4 & 44.1 & 45.6 & 45.6 & 0.1 & 0.1 & 0.1 \\
$R_{t}$ & Nominal interest & 46.7 & 45.9 & 47.3 & 46.0 & 47.2 & 46.2 & 5.5 & 5.8 & 5.5 \\
$c_{t}-\log \Phi_{t}$ & Consumption & 58.0 & 61.9 & 70.7 & 6.0 & 6.8 & 4.7 & 0.3 & 0.3 & 0.2 \\
$i_{t}-\log \left(\Phi_{t} \Psi_{t}\right)$ & Investment & 70.2 & 72.1 & 81.4 & 21.7 & 23.7 & 18.3 & 0.3 & 0.4 & 0.2 \\
$\eta_{t}$ & Hiring rate & 38.2 & 38.0 & 42.9 & 38.9 & 40.4 & 33.8 & 1.1 & 1.1 & 0.9 \\
$V_{t}-\log \Phi_{t}$ & Continuation value & 67.3 & 68.7 & 74.0 & 6.9 & 7.6 & 5.0 & 0.2 & 0.2 & 0.1 \\
$\bar{\Delta}_{t}^{(4)}(d \mathcal{Y})$ & GDP growth wedge & - & - & - & - & - & - & - & - & - \\
$\bar{\Delta}_{t}^{(4)}(u)$ & Unemp. wedge & - & - & - & - & - & - & - & - & - \\
$\bar{\Delta}_{t}^{(4)}(\pi)$ & Inflation wedge & - & - & - & - & - & - & - & - & - \\
$\widetilde{E}_{t} \log \left(\mathcal{Y}_{t+1} / \mathcal{Y}_{t}\right)$ & GDP growth forecast & - & - & 28.8 & - & - & 37.8 & - & - & 0.2 \\
$\widetilde{E}_{t} u_{t+4}$ & Unemp. forecast & - & - & 46.3 & - & - & 39.6 & - & - & 0.2 \\
$\widetilde{E}_{t} \pi_{t+4}$ & Inflation forecast & - & - & 57.3 & - & - & 32.5 & - & - & 0.1
\end{tabular}

\begin{tabular}{ll|rrr|rrr} 
Variable & & \multicolumn{3}{|c|}{$w^{f}$} & \multicolumn{3}{|c}{ meas. error } \\
& & base & med & mod & base & med & mod \\
\hline $\log \left(\mathcal{Y}_{t} / \mathcal{Y}_{t-1}\right)$ & GDP growth & 24.8 & 23.5 & 25.0 & - & - & - \\
$u_{t}$ & Unemployment & 13.6 & 12.2 & 14.0 & - & - & - \\
$\pi_{t}$ & Inflation & 2.6 & 2.1 & 1.9 & - & - & - \\
$R_{t}$ & Nominal interest & 1.9 & 1.2 & 1.0 & - & - & - \\
$c_{t}-\log \Phi_{t}$ & Consumption & 35.7 & 31.0 & 24.5 & - & - & - \\
$i_{t}-\log \left(\Phi_{t} \Psi_{t}\right)$ & Investment & 7.8 & 3.8 & 0.1 & - & - & - \\
$\eta_{t}$ & Hiring rate & 21.8 & 20.6 & 22.4 & - & - & - \\
$V_{t}-\log \Phi_{t}$ & Continuation value & 25.6 & 23.5 & 20.9 & - & - & - \\
$\bar{\Delta}_{t}^{(4)}(d \mathcal{Y})$ & GDP growth wedge & 16.4 & 15.1 & 6.9 & 83.6 & 84.9 & 93.1 \\
$\bar{\Delta}_{t}^{(4)}(u)$ & Unemp. wedge & 6.3 & 5.3 & 2.5 & 93.7 & 94.7 & 97.5 \\
$\bar{\Delta}_{t}^{(4)}(\pi)$ & Inflation wedge & 22.0 & 19.9 & 9.1 & 78.0 & 80.1 & 90.9 \\
$\widetilde{E}_{t} \log \left(\mathcal{Y}_{t+4} / \mathcal{Y}_{t}\right)$ & GDP growth forecast & - & - & 11.4 & - & - & 21.9 \\
$\widetilde{E}_{t} u_{t+4}$ & Unemp. forecast & - & - & 13.2 & - & - & 0.7 \\
$\widetilde{E}_{t} \pi_{t+4}$ & Inflation forecast & - & - & 1.6 & - & - & 8.5
\end{tabular}

Table 12: Structural model variance decomposition using estimates from alternative measures of wedges. The column 'base' uses wedges defined as the difference in the mean responses of the household and professional forecasters, the column 'med' computes the wedges using the median household survey responses, and the column 'mod' computes the wedges relative to the model-implied forecasts. 

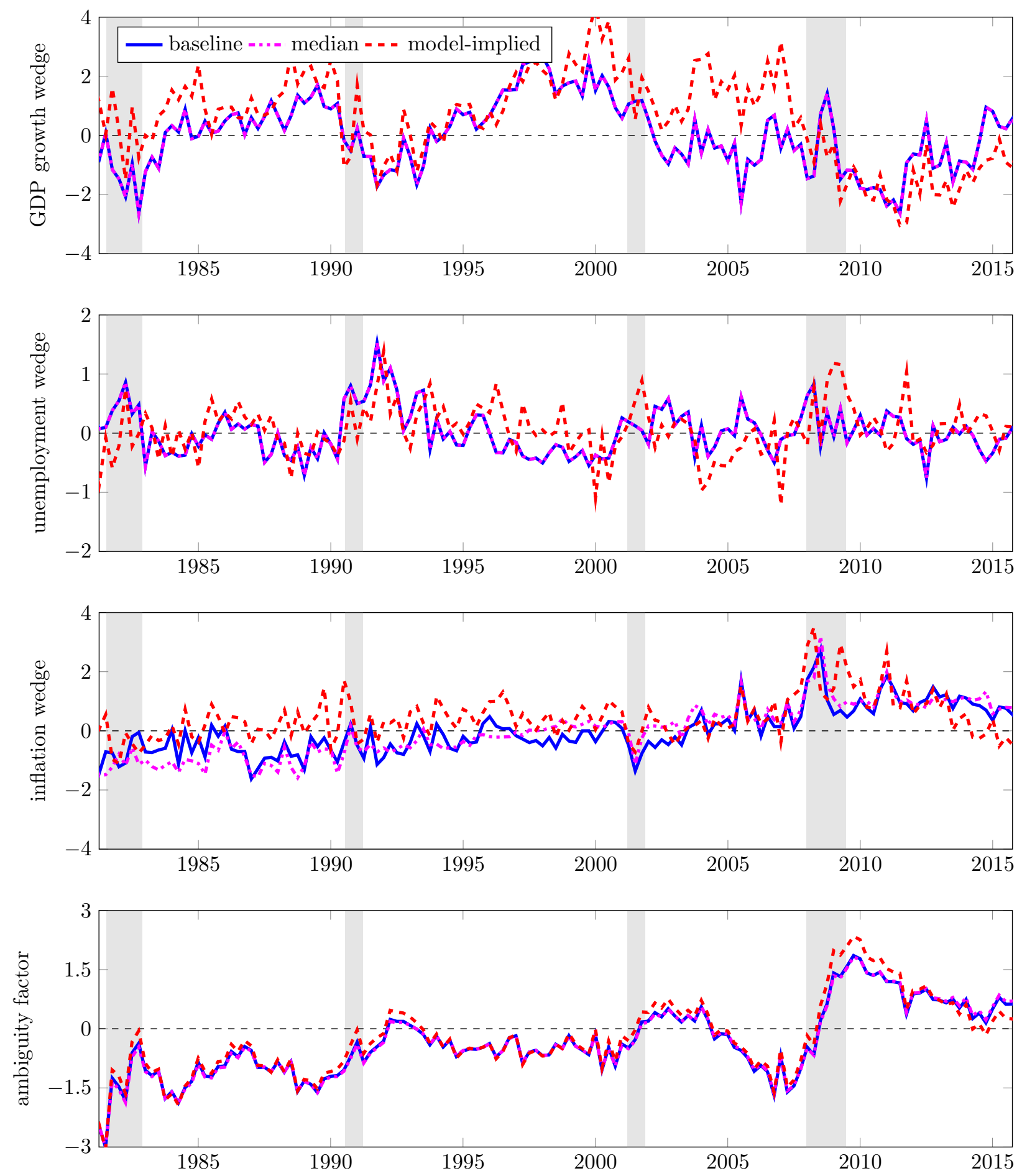

Figure 12: Comparison across structural model specifications with alternative measures of belief wedges. The first three panels are the smoothed time-series for the GDP growth wedge, unemployment wedge and inflation wedge. All wedges are annualized and demeaned. The last panel is the smoothed time-series for the standardized factor. In all panels, the 'baseline' specification uses wedges defined as the difference in the mean responses of the household and professional forecasters, 'median' computes the wedges using the median household survey responses, and 'model-implied' computes the wedges relative to the model-implied forecasts. 


\section{References}

Adam, Klaus and Michael Woodford. 2012. Robustly Optimal Monetary Policy in a Microfounded New Keynesian Model. Journal of Monetary Economics 59 (5):468-487.

Anderson, Evan W., Lars Peter Hansen, and Thomas J. Sargent. 2003. A Quartet of Semigroups for Model Specification, Robustness, Prices of Risk, and Model Detection. Journal of the European Economic Association 1 (1):68-123.

Ang, Andrew and Monika Piazzesi. 2003. A No-Arbitrage Vector Autoregression of Term Structure Dynamics with Macroeconomic and Latent Variables. Journal of Monetary Economics $50(4): 745-787$.

Ang, Andrew, Geert Bekaert, and Min Wei. 2007. Do Macro Variables, Asset Markets, or Surveys Forecasts Inflation Better? Journal of Monetary Economics 54 (4):1163-1212.

Bachmann, Rüdiger, Steffen Elstner, and Eric R. Sims. 2012. Uncertainty and Economic Activity: Evidence from Business Survey Data. American Economic Journal: Macroeconomics 5 (2):217249 .

Barillas, Francisco, Lars Peter Hansen, and Thomas J. Sargent. 2009. Doubts or Variability? Journal of Economic Theory 144 (6):2388-2418.

Barsky, Robert B. and Eric R. Sims. 2012. Information, Animal Spirits, and the Meaning of Innovations in Consumer Confidence. American Economic Review 102 (4):1343-1377.

Beaudry, Paul and Franck Portier. 2004. An Exploration into Pigou's Theory of Cycles. Journal of Monetary Economics 51 (6):1183-1216.

Bernanke, Ben S., Jean Boivin, and Piotr Eliasz. 2005. Measuring the Effects of Monetary Policy: A Factor-Augmented Vector Autoregressive (FAVAR) Approach. The Quarterly Journal of Economics 120 (1):387-422.

Bhandari, Anmol. 2015. Doubts, Asymmetries, and Insurance. Job market paper.

Bianchi, Francesco, Cosmin Ilut, and Martin Schneider. 2014. Uncertainty Shocks, Asset Supply and Pricing over the Business Cycle.

Bidder, Rhys and Matthew E. Smith. 2012. Robust Animal Spirits. Journal of Monetary Economics 59 (8):738-750.

Binmore, Ken, Ariel Rubinstein, and Asher Wolinsky. 1986. The Nash Bargaining Solution in Economic Modelling. RAND Journal of Economics 17 (2):176-188.

Blanchard, Olivier Jean and Charles M. Kahn. 1980. The Solution of Linear Difference Models under Rational Expectations. Econometrica 48 (5):1305-1312. 
Borovička, Jaroslav and Lars Peter Hansen. 2013. Robust Preference Expansions.

- 2014. Examining Macroeconomic Models through the Lens of Asset Pricing. Journal of Econometrics 183 (1):67-90.

Cagetti, Marco, Lars Peter Hansen, Thomas J. Sargent, and Noah Williams. 2002. Robustness and Pricing with Uncertain Growth. The Review of Financial Studies 15 (2):363-404.

Calvo, Guillermo A. 1983. Staggered Prices in a Utility-Maximizing Framework. Journal of Monetary Economics 12 (3):383-398.

Capistrán, Carlos and Allan Timmermann. 2009. Disagreement and Biases in Inflation Expectations. Journal of Money, Credit and Banking 41 (2-3):365-396.

Carlson, John A. and Michael Parkin. 1975. Inflation Expectations. Economica 42 (166):123-138.

Carroll, Christopher D. 2003. Macroeconomic Expectations of Households and Professional Forecasters. Quarterly Journal of Economics 118 (1):269-298.

Christiano, Lawrence J., Martin S. Eichenbaum, and Mathias Trabandt. 2015. Unemployment and Business Cycles. Forthcoming in Econometrica.

Coibion, Olivier and Yuriy Gorodnichenko. 2012. What Can Survey Forecasts Tell Us about Information Rigidities? Journal of Political Economy 120 (1):116-159.

- 2015. Is the Phillips Curve Alive and Well After All? Inflation Expectations and the Missing Disinflation. American Economic Journal: Macroeconomics 7 (1):197-232.

Croushore, Dean. 2010. An Evaluation of Inflation Forecasts from Surveys Using Real-Time Data. B.E. Journal of Macroeconomics 10 (1).

Elliott, Graham, Ivana Komunjer, and Allan Timmermann. 2008. Biases in Macroeconomic Forecasts: Irrationality or Asymmetric Loss? Journal of the European Economic Association $6(1): 122-157$.

Epstein, Larry G. and Martin Schneider. 2003. Recursive Mutiple-Priors. Journal of Economic Theory 113 (1):1-31.

—. 2007. Learning under Ambiguity. Review of Economic Studies 74 (4):1275-1303.

- 2010. Ambiguity and Asset Markets. Annual Review of Financial Economics 2 (1):315-346.

Epstein, Larry G. and Stanley E. Zin. 1989. Substitution, Risk Aversion, and the Temporal Behavior of Consumption and Asset Returns: A Theoretical Framework. Econometrica 57 (4):937-969.

Faust, Jon and Jonathan H. Wright. 2013. Forecasting Inflation. In Handbook of Economic Forecasting: Volume 2A, chap. 1, 3-56. Elsevier B.V. 
Gilboa, Itzhak and David Schmeidler. 1989. Maxmin Expected Utility with Non-Unique Prior. Journal of Mathematical Economics 18 (2):141-153.

Hall, Robert E. 2015. High Discounts and High Unemployment. Stanford University.

Hall, Robert E. and Paul R. Milgrom. 2008. The Limited Influence of Unemployment on the Wage Bargain. American Economic Review 98 (4):1653-1674.

Hansen, Lars Peter and Thomas J. Sargent. 2001a. Acknowledging Misspecification in Macroeconomic Theory. Review of Economic Dynamics 4 (3):519-535.

—. 2001b. Robust Control and Model Uncertainty. The American Economic Review 91 (2):6066.

2007. Recursive Robust Estimation and Control without Commitment. Journal of Economic Theory 136 (1):1-27.

- 2010. Fragile Beliefs and the Price of Uncertainty. Quantitative Economics 1 (1):129-162.

- 2011. Wanting Robustness in Macroeconomics. In Handbook of Monetary Economics, Volume 3B, chap. 20, 1097-1157. Elsevier B.V.

—. 2012. Three Types of Ambiguity. Journal of Monetary Economics 59 (5):422-445.

- 2015. Sets of Models and Prices of Uncertainty. Working paper, University of Chicago and New York University.

Holmes, Mark H. 1995. Introduction to Perturbation Methods. Springer.

Ilut, Cosmin and Martin Schneider. 2014. Ambiguous Business Cycles. American Economic Review 104 (8):2368-2399.

Ju, Nengjiu and Jianjun Miao. 2012. Ambiguity, Learning, and Asset Returns. Econometrica 80 (2):559-591.

Jurado, Kyle. 2015. Advance Information and Distorted Beliefs in Macroeconomics and Financial Fluctuations. Job market paper.

Karantounias, Anastasios G. 2013. Managing Pessimistic Expectations and Fiscal Policy. Theoretical Economics 8 (1):193-231.

Klibanoff, Peter, Massimo Marinacci, and Sujoy Mukerji. 2005. A Smooth Model of Decision Making under Ambiguity. Econometrica 73 (6):1849-1892.

. 2009. Recursive Smooth Ambiguity Preferences. Journal of Economic Theory 144 (3):930976. 
Kwon, Hyosung and Jianjun Miao. 2013. Three Types of Robust Ramsey Problems in a LinearQuadratic Framework. October.

Lombardo, Giovanni. 2010. On Approximating DSGE Models by Series Expansions. ECB Working paper No. 1264.

Mankiw, N. Gregory, Ricardo Reis, and Justin Wolfers. 2003. Disagreement about Inflation Expectations. NBER Macroeconomics Annual 18:209-248.

Orlik, Anna and Ignacio Presno. 2013. On Credible Monetary Policies with Model Uncertainty.

Piazzesi, Monika, Juliana Salomao, and Martin Schneider. 2015. Trend and Cycle in Bond Premia.

Pigou, Arthur C. 1927. Industrial Fluctuations. MacMillan, London.

Ravenna, Federico and Carl E. Walsh. 2008. Vacancies, Unemployment, and the Phillips Curve. European Economic Review 52 (8):1494-1521.

Reis, Ricardo. 2009. Optimal Monetary Policy Rules in an Estimated Sticky. American Economic Journal: Macroeconomics 1 (2):1-28.

Rubinstein, Ariel. 1982. Perfect Equilibrium in a Bargaining Model. Econometrica 50 (1):97-109.

Schmitt-Grohé, Stephanie and Martín Uribe. 2004. Solving Dynamic General Equilibrium Models Using a Second-Order Approximation to the Policy Function. Journal of Economic Dynamics and Control 28 (4):755-775.

Sims, Christopher A. 2002. Solving Rational Expectations Models. Computational Economics $20(1-2): 1-20$.

Strzalecki, Tomasz. 2011. Axiomatic Foundations of Multiplier Preferences. Econometrica 79 (1):47-73.

Thomas, Jr., Lloyd B. 1999. Survey Measures of Expected U.S. Inflation. Journal of Economic Perspectives 13 (4):125-144. 